\title{
HOMOGENIZATION-LIMIT OF A MODEL SYSTEM FOR INTERACTION OF FLOW, CHEMICAL REACTIONS AND MECHANICS IN CELL TISSUES
}

\author{
WILLI JÄGER*, ANDRO MIKELIĆ ${ }^{\dagger}$, AND MARIA NEUSS-RADU $\ddagger$
}

\begin{abstract}
In this article we obtain rigorously the homogenization limit for a fluid-structurereactive flow system. It consists of cell tissue and intercellular liquid, transporting solutes. The cell tissue is supposed linearly elastic and deforming with a viscous non-stationary flow. The elastic moduli of the tissue change with cumulative concentration value. In the limit, when the scale parameter goes to zero, we obtain the quasi-static Biot system, coupled with the upscaled reactive flow. Effective Biot's coefficients depend on the reactant concentration. Additionally to the weak two-scale convergence results, we prove convergence of the elastic and viscous energies. This then implies a strong two-scale convergence result for the fluid-structure variables. Next we establish the regularity of the solutions for the upscaled equations. In our knowledge, it is the only known study of the regularity of solutions to the quasi-static Biot system. The regularity is used to prove the uniqueness for the upscaled model.
\end{abstract}

Key words. reactive transport, fluid-structure interaction, homogenization, biological-tissue, generalized quasi-static Biot system.

AMS subject classifications. 93A30, 35Q30, 47F10, 35B27, 92C50

1. Introduction. Information on biophysical and biochemical processes on all scales has enormously increased due to a revolution in experimental concepts and technologies. Consequently, quantitative methods, based on mathematical modeling and simulations are becoming more and more important in analyzing experimental data and designing theories based on mathematical concepts. One of the numerous challenges is modeling processes in tissues, including the molecular information on micro-scale. In this paper, the following processes in cell tissues are included:

1. fluid flow in the extracellular space, diffusion, transport and reactions of substances in the fluid,

2. exchange of substances at the cell membranes,

3. diffusion of substances and chemical reactions inside the cells,

4. changes of the mechanical properties of the cells due to the influence of chemical substances, small deformations of the structure.

The corresponding microscopic system was formulated and analyzed by the authors in [10], where the existence and uniqueness of solutions was proven. Also in [10], the characteristic microscopic scale $\varepsilon$ of this system was identified, depending on the application in cell biology and the available real data.

In this paper, the scale limit $\varepsilon \rightarrow 0$ is performed. For simplicity, the structure of the tissue is assumed to be periodic, that means generated by translations of a properly scaled geometric unit cell containing a biological cell connected with its adjacent neighbor cells. Here, fluid flow is restricted to the intercellular region $\Omega_{f}^{\varepsilon}$ (fluid region) and interacting with the cell region $\Omega_{s}^{\varepsilon}$ (solid region). A chemical substance is diffusing

*IWR, University of Heidelberg, INF 368, 69120 Heidelberg, Germany Phone: +49 6221548235 (jaeger@iwr.uni-heidelberg.de).

†Université de Lyon, F-69003 France, Université Lyon 1, Institut Camille Jordan,Site de Gerland, Bât. A, 50, avenue Tony Garnier, 69367 Lyon Cedex 07, France Phone: (Andro.Mikelic@univlyon1.fr).

${ }^{\ddagger}$ IWR, University of Heidelberg, INF 368, 69120 Heidelberg, Germany, Phone: +49 6221546187 (maria.neuss-radu@iwr.uni-heidelberg.de). 
in the full domain $\Omega$ and is transported by the fluid flow in $\Omega_{f}^{\varepsilon}$. In the interior of the cells, the substance is reacting with an other substance, which on its part interacts with the mechanical structure of the cell. It is assumed that the elasticity parameters depend on this substance via a Volterra-functional of its concentration.

Using rigorous multiscale techniques, the homogenization limit is performed, and a macroscopic (effective) model system is derived preserving relevant information on the processes on the microscopic level. A set of estimates of the solutions to the microscopic system and their dependence on the scale parameter $\varepsilon$ is derived, allowing to apply the method of two-scale convergence.

In the limit the quasi-static diphasic Biot system for the fluid-structure part is obtained. Effective phase displacements, velocities and Biot's pressure are kept, but the acceleration terms are not present in the effective model. Its particular property is the dependence on the concentration of transported reactant. At the other hand, the homogenized reactive flow equations contain the transport velocity coming from Biot's diphasic system. The full homogenized system is presented in Section 3.2. This system seems to be new in the literature, and we prove the corresponding regularity and uniqueness results. We note that, in general, quasi-static elasticity problems do not have unique solution (see e.g. result by Klarbring in [11]). Besides weak twoscale convergence results, we prove convergence of the energies, which then allow us to derive strong two-scale convergence results for the fluid-structure variables, see Theorem 3.1, and Corollary 3.2.

These investigations were motivated by questions asked by physiologists interested in perfusion an transport through tissue under varying mechanical and chemical conditions. The effective permeability of the porous media is changed under the influence of the mechanical changes in the solid phase. Note that for a flow through an elastic pore the permeability depends on the pressure (see e.g. argument in [9]). Experimental studies were performed by [15] for thin layers of endothelial cells. These cell layers were exposed either to chemicals or to shear stress caused by flow. The transmission through the membrane was measured and our results give a mathematical model which could be used to explain the observations.

The interaction of fluid with solid structures has been studied in the literature in several papers and passing to the homogenization limit the macroscopic law known as Biot-law could be derived, see [6], [7], [8], [16]. The system, which is analyzed here represents a larger class of problems coupling fluid flow, solid structure and chemical reaction for slow flow velocity. Its study requires new ideas and methods which are developed in this paper. The authors are not aware of mathematical results for systems of this type.

This paper is organized as follows: In Section 2, the model system is formulated. The assumptions on the data and the main results of the paper are formulated in Section 3. Estimates of the solutions with explicit dependence on the scale parameter $\varepsilon$ are formulated and derived in Section 4.3. In Section 5, the convergence is analyzed and in Section 6 the limit equations are derived. We prove convergence for the energies of the fluid-structure variables in Section 7. This result then implies strong two-scale convergence for the homogenized displacement and velocity. Next, we establish higher regularity for the variational solutions of the homogenized equations. The regularity is used in Section 9 to prove uniqueness of the solutions of the effective model.

2. Setting of the model. Let $\varepsilon>0$ be a sequence of strictly positive numbers tending to zero, with the property that $\frac{1}{\varepsilon} \in \mathbb{N}$. Let $[0, T]$ denote a time interval, with $T>0$. We consider the domain $\Omega=(0,1)^{3}$ consisting of two subdomains: the tissue 
part formed by the elastic cells and the fluid part representing the intercellular space, see Fig 2.1. The tissue part is denoted by $\Omega_{s}^{\varepsilon}$, the fluid part by $\Omega_{f}^{\varepsilon}$, and the fluid-solid interface by $\Gamma^{\varepsilon}=\partial \Omega_{f}^{\varepsilon} \cap \partial \Omega_{s}^{\varepsilon}$. The boundary of the domain $\Omega$ consists of three parts

$$
\partial \Omega=\Gamma_{1} \cup \Gamma_{2} \cup \Gamma_{3},
$$

where $\Gamma_{1}=\left\{x_{1}=0\right\} \times[0,1]^{2}, \Gamma_{2}=\left\{x_{1}=1\right\} \times[0,1]^{2}$ and $\Gamma_{3}=\cup_{j=2,3}\left(\left\{x_{j}=0\right\} \cup\left\{x_{j}=\right.\right.$ $1\}) \times(0,1)^{2}$. The outer unit normal to $\partial \Omega$ is denoted by $\nu$. On the interface $\Gamma^{\varepsilon}$, we denote by $\nu$ the outer unit normal to the solid part $\Omega_{s}^{\varepsilon}$. The microscopic structure of

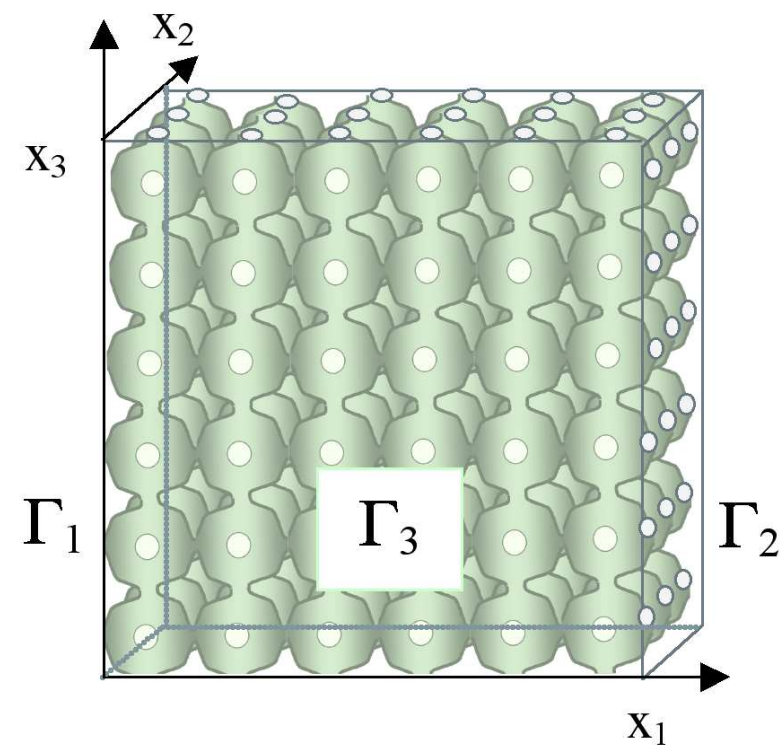

FIG. 2.1. The domain $\Omega$ with the components $\Omega_{s}^{\varepsilon}$ and $\Omega_{f}^{\varepsilon}$.

$\Omega_{s}^{\varepsilon}$ and $\Omega_{f}^{\varepsilon}$ is periodic, and is obtained by the repetition of the standard cell $Y=(0,1)^{3}$ scaled with the small parameter $\varepsilon$, see also Fig. 2.1. We denote by $Y_{f}$ and $Y_{s}$ the fluid respectively the solid part of $Y$. The outer unit normal to $\partial Y_{s}$ is also denoted by $\nu$. Furthermore, let $F_{f}$, and $F_{s}$ be the fluid respectively the solid part of the face $F_{1}$ of the unit cell Y, i.e.

$$
F_{1}=\bar{Y} \cap\left\{y_{1}=1\right\}, \quad F_{f}=\bar{Y}_{f} \cap\left\{y_{1}=1\right\}, \quad \text { and } \quad F_{s}=\bar{Y}_{s} \cap\left\{y_{1}=1\right\} .
$$

We assume that

1. $Y=\bar{Y}_{s} \cup Y_{f}$.

2. $Y_{f}$ and $Y_{s}$ are open, connected sets of strictly positive measure in $Y$, with Lipschitz boundary.

3. The sets

$$
E_{s}:=\bigcup_{k \in \mathbb{Z}^{3}} Y_{s}^{k}=\bigcup_{k \in \mathbb{Z}^{3}}\left(k+Y_{s}\right) \text { and } E_{f}:=\bigcup_{k \in \mathbb{Z}^{3}} Y_{f}^{k}=\bigcup_{k \in \mathbb{Z}^{3}}\left(k+Y_{f}\right)
$$

are open, connected, and have Lipschitz boundary.

Then, we define

$$
\Omega_{s}^{\varepsilon}=\Omega \cap \varepsilon E_{s}, \quad \text { and } \quad \Omega_{f}^{\varepsilon}=\Omega \cap \varepsilon E_{f} .
$$


Throughout the paper we denote by $\mathbf{e}_{j}$ the $\mathrm{j}$-th unit vector in $\mathbb{R}^{3}$.

Let us now formulate the equations which model the processes at the microscopic level. We suppose small deformations of the cell structure. It means that in the solid part $\Omega_{s}^{\varepsilon}$ the equations of linear elasticity hold. The interaction of the chemical substance with the mechanical properties of the cells is modeled by considering elasticity coefficients $A$ which depend on the concentration $c_{1}^{\varepsilon}$ of chemical substance. Thus we have

$$
\rho_{s} \frac{\partial^{2} \mathbf{u}^{\varepsilon}}{\partial t^{2}}-\nabla \cdot\left(\sigma\left(\mathbf{u}^{\varepsilon}\right)\right)=0 \text { in } \Omega_{s}^{\varepsilon} \times(0, T)
$$

where $\mathbf{u}^{\varepsilon}$ is the microscopic displacement, $D\left(\mathbf{u}^{\varepsilon}\right)$ is the strain tensor, and $\sigma\left(\mathbf{u}^{\varepsilon}\right)$ is the stress tensor given by

$$
\sigma\left(\mathbf{u}^{\varepsilon}\right)=A\left(\mathcal{F}\left(c_{1}^{\varepsilon}\right)\right) D\left(\mathbf{u}^{\varepsilon}\right)
$$

If the cells are considered as homogeneous and isotropic bodies, the elasticity coefficients $A$ are given with the help of Lamé's coefficients $\lambda$ and $\mu$ and the stress tensor has the following form

$$
\sigma\left(\mathbf{u}^{\varepsilon}\right)=\lambda\left(\mathcal{F}\left(c_{1}^{\varepsilon}\right)\right) \nabla \cdot\left(\mathbf{u}^{\varepsilon} I\right)+2 \mu\left(\mathcal{F}\left(c_{1}^{\varepsilon}\right)\right) D\left(\mathbf{u}^{\varepsilon}\right) .
$$

The dependence of the elasticity coefficients on the concentration $c_{1}^{\varepsilon}$ is nonlinear and nonlocal; the coefficients change as a function of cumulated quantity of chemical substance. To describe this dependence, we introduce the operator $\mathcal{F}$ acting on the concentration, and given by

$$
\begin{gathered}
\mathcal{F}: L^{2}\left(\Omega_{s}^{\varepsilon} \times[0, T]\right) \rightarrow L^{2}\left(\Omega_{s}^{\varepsilon} \times[0, T]\right) \\
\mathcal{F}\left(c_{1}^{\varepsilon}\right)(x, t)=\left(\mathcal{K} \star_{t} F\left(c_{1}^{\varepsilon}\right)\right)(x, t)=\int_{0}^{t} \mathcal{K}(t-\tau) F\left(c_{1}^{\varepsilon}(x, \tau)\right) d \tau,
\end{gathered}
$$

where $F \in C^{2}(\mathbb{R})$ is Lipschitz, and the kernel $\mathcal{K}$ has the following properties

$$
\mathcal{K} \in C^{3}[0, T], \quad \mathcal{K}(0)=\mathcal{K}^{\prime}(0)=\mathcal{K}^{\prime \prime}(0)=0 .
$$

In the intercellular space $\Omega_{f}^{\varepsilon}$, we consider the linearized Navier-Stokes system for a viscous and incompressible fluid. The interface between the tissue and liquid is also linearized. This simplified equations for the flow are obtained in [10] by a dimensional analysis based on a set of characteristic values for the physical parameters. In its dimensionless form, the fluid-structure interaction is described by means of the microscopic displacement function $\mathbf{u}^{\varepsilon}$, and the fluid pressure $p^{\varepsilon}$. It has the following 
form (for a detailed description we refer to [10]).

$$
\begin{aligned}
& \frac{\partial^{2} \mathbf{u}^{\varepsilon}}{\partial t^{2}}+\frac{1}{\varepsilon^{2}} \nabla p^{\varepsilon}=\Delta\left(\frac{\partial \mathbf{u}^{\varepsilon}}{\partial t}\right) \text { in } \Omega_{f}^{\varepsilon} \times(0, T) \\
& \nabla \cdot\left(\frac{\partial \mathbf{u}^{\varepsilon}}{\partial t}\right)=0 \text { in } \Omega_{f}^{\varepsilon} \times(0, T) \\
& \frac{\partial^{2} \mathbf{u}^{\varepsilon}}{\partial t^{2}}=\frac{1}{\varepsilon^{2}} \nabla \cdot\left(A D\left(\mathbf{u}^{\varepsilon}\right)\right) \text { in } \Omega_{s}^{\varepsilon} \times(0, T) \\
& \mathbf{u}^{\varepsilon} \chi_{\Omega_{f}^{\varepsilon}}=\mathbf{u}^{\varepsilon} \chi_{\Omega_{s}^{\varepsilon}} \text { on } \Gamma^{\varepsilon} \times(0, T) \\
& \left(-\frac{1}{\varepsilon^{2}} p^{\varepsilon} I+2 D\left(\frac{\partial \mathbf{u}^{\varepsilon}}{\partial t}\right)\right) \cdot \nu=\frac{1}{\varepsilon^{2}} A D\left(\mathbf{u}^{\varepsilon}\right) \cdot \nu \text { on } \Gamma^{\varepsilon} \times(0, T) \\
& \left(-\frac{1}{\varepsilon^{2}} p^{\varepsilon} I+2 D\left(\frac{\partial \mathbf{u}^{\varepsilon}}{\partial t}\right)\right) \cdot \mathbf{e}_{1}= \\
& =\left\{\begin{array}{ccc}
0 & \text { on } & \left(\Gamma_{1} \cap \bar{\Omega}_{f}^{\varepsilon}\right) \times(0, T) \\
\frac{1}{\varepsilon^{2}}\left(\mathcal{P}_{1}^{\varepsilon}, \mathcal{S}_{2}^{\varepsilon}, \mathcal{S}_{3}^{\varepsilon}\right) & \text { on } & \left(\Gamma_{2} \cap \bar{\Omega}_{f}^{\varepsilon}\right) \times(0, T)
\end{array}\right. \\
& \frac{1}{\varepsilon^{2}}\left(A D\left(\mathbf{u}^{\varepsilon}\right)\right) \cdot \mathbf{e}_{1}=\left\{\begin{array}{ccc}
0 & \text { on } & \left(\Gamma_{1} \cap \bar{\Omega}_{s}^{\varepsilon}\right) \times(0, T) \\
\frac{1}{\varepsilon^{2}}\left(\mathcal{P}_{1}^{\varepsilon}, \mathcal{S}_{2}^{\varepsilon}, \mathcal{S}_{3}^{\varepsilon}\right) & \text { on } & \left(\Gamma_{2} \cap \bar{\Omega}_{s}^{\varepsilon}\right) \times(0, T)
\end{array}\right. \\
& \frac{\partial \mathbf{u}^{\varepsilon}}{\partial t} \chi_{\Omega_{f}^{\varepsilon}}+\mathbf{u}^{\varepsilon} \chi_{\Omega_{s}^{\varepsilon}}=0 \text { on } \Gamma_{3} \times(0, T) \\
& \mathbf{u}^{\varepsilon}(x, 0)=0, \quad \frac{\partial \mathbf{u}^{\varepsilon}}{\partial t}(x, 0)=0 \text { in } \Omega
\end{aligned}
$$

We remark that the size of the elastic modulii and of the characteristic fluid pressure is of order $O\left(1 / \varepsilon^{2}\right)$, whereas the viscosity forces are of order $O(1)$. See [10] for more details.

Next, we have the equations describing the distribution of the chemical substances involved in our model. There is one chemical with concentration $c_{1}^{\varepsilon}$ diffusing only inside cells and interacting with the elastic cell structure, and a second chemical, which is present in the cells and in the intercellular space, and influencing diffusion and reactive change of the first chemical substance. We remark that the concentration $c_{2}^{\varepsilon}$ of the second chemical substance is discontinuous at the interface $\Gamma^{\varepsilon}$. In order to work with the usual Sobolev spaces we redefine $c_{2}^{\varepsilon}$ on the solid part, such that the concentration is continuous at the interface. However, this transformation then leads to discontinuities in the coefficients of the equation for $c_{2}^{\varepsilon}$. For details concerning this 
transformation, see again [10].

$$
\begin{aligned}
& \frac{\partial c_{1}^{\varepsilon}}{\partial t}-\nabla \cdot\left(d_{1}\left(c_{2}^{\varepsilon}\right) \nabla c_{1}^{\varepsilon}\right)=g_{1}\left(c_{1}^{\varepsilon}, c_{2}^{\varepsilon}\right) \text { in } \Omega_{s}^{\varepsilon} \times(0, T) \\
& d_{1}\left(c_{2}^{\varepsilon}\right) \nabla c_{1}^{\varepsilon} \cdot \nu=0 \text { on } \partial \Omega_{s}^{\varepsilon} \times(0, T) \\
& c_{1}^{\varepsilon}(0)=c_{10} \text { in } \Omega_{s}^{\varepsilon} \\
& \frac{\partial c_{2}^{\varepsilon}}{\partial t}+\frac{\partial \mathbf{u}^{\varepsilon}}{\partial t} \cdot \nabla c_{2}^{\varepsilon}-d_{2} \Delta c_{2}^{\varepsilon}=g_{2}\left(c_{2}^{\varepsilon}\right) \text { in } \Omega_{f}^{\varepsilon} \times(0, T) \\
& \frac{1}{K} \frac{\partial c_{2}^{\varepsilon}}{\partial t}-\frac{1}{K} d_{2} \Delta c_{2}^{\varepsilon}=g_{3}\left(c_{1}^{\varepsilon}, c_{2}^{\varepsilon}\right) \text { in } \Omega_{s}^{\varepsilon} \times(0, T) \\
& \left(\frac{\partial \mathbf{u}^{\varepsilon}}{\partial t} c_{2}^{\varepsilon}-d_{2} \nabla c_{2}^{\varepsilon}\right) \chi_{\Omega_{f}^{\varepsilon}} \cdot \nu=-\frac{d_{2}}{K} \nabla c_{2}^{\varepsilon} \chi_{\Omega_{s}^{\varepsilon}} \cdot \nu \text { on } \Gamma^{\varepsilon} \times(0, T) \\
& c_{2}^{\varepsilon} \chi_{\Omega_{f}^{\varepsilon}}=c_{2}^{\varepsilon} \chi_{\Omega_{s}^{\varepsilon}} \text { on } \Gamma^{\varepsilon} \times(0, T) \\
& c_{2}^{\varepsilon} \chi_{\Omega_{f}^{\varepsilon}}+c_{2}^{\varepsilon} \chi_{\Omega_{s}^{\varepsilon}}=c_{2 D} \text { on }\left(\Gamma_{1} \cup \Gamma_{2}\right) \times(0, T) \\
& \left(\frac{\partial \mathbf{u}^{\varepsilon}}{\partial t} c_{2}^{\varepsilon}-d_{2} \nabla c_{2}^{\varepsilon}\right) \chi_{\Omega_{f}^{\varepsilon}} \cdot \nu-\frac{d_{2}}{K} \nabla c_{2}^{\varepsilon} \chi_{\Omega_{s}^{\varepsilon}} \cdot \nu=0 \text { on } \Gamma_{3} \times(0, T) \\
& \left(c_{2}^{\varepsilon} \chi_{\Omega_{f}^{\varepsilon}}+c_{2}^{\varepsilon} \chi_{\Omega_{s}^{\varepsilon}}\right)(0)=c_{20} \text { in } \Omega .
\end{aligned}
$$

In the equations for the concentrations, the nonlinear terms $g_{i}$ represent chemical reactions rates and $d_{i}$ are diffusion coefficients.

Let for a given bounded domain $G \subset \mathbb{R}^{3}, W^{m, q}(G), 1 \leq q \leq+\infty, m \in \mathbb{N}$, denote the Sobolev space of functions from $L^{q}(G)$ having derivatives of order $m$ in $L^{q}(G)$. For $q=2$, these spaces are denoted by $H^{m}(G)$. We also use the spaces of functions depending on space and time $W_{q}^{2 \ell, \ell}(G \times(0, T)), \ell>0$, consisting of functions having derivatives with respect to space up to order $2 \ell$ and with respect to time up to order $\ell$ in $L^{q}$. For the precise definition of these spaces see [13].

In [10], the existence, uniqueness and stability of a solution $\left(\mathbf{u}^{\varepsilon}, c_{1}^{\varepsilon}, c_{2}^{\varepsilon}\right)$, with

$$
\begin{gathered}
\mathbf{u}^{\varepsilon} \in W^{3, \infty}\left(0, T ; L^{2}(\Omega)\right) \cap W^{2, \infty}\left(0, T ; H^{1}(\Omega)\right) \cap H^{3}\left(0, T ; H^{1}\left(\Omega_{f}^{\varepsilon}\right)\right), \\
c_{1}^{\varepsilon} \in W_{2}^{2,1}\left(Q_{T}^{s}\right), \quad \text { and } \quad c_{2}^{\varepsilon} \in W_{2}^{1,1}(Q) \cap W_{2}^{2,1}\left(Q_{T}^{s} \cup Q_{T}^{f}\right),
\end{gathered}
$$

of problem (2.9)-(2.27), has been proven, under the assumptions on the data given in the next section. Here we used the notations

$$
Q=\Omega \times(0, T), \quad Q_{T}^{s}=\Omega_{s}^{\varepsilon} \times(0, T), \quad Q_{T}^{f}=\Omega_{f}^{\varepsilon} \times(0, T) .
$$

We complete this section by the variational formulation of the microscopic problems. This formulation will be the starting point in proving estimates for the microscopic solutions, and also for performing the homogenization limit $\varepsilon \rightarrow 0$. 
2.1. Variational formulation of the microscopic problems. Find $\left(\mathbf{u}^{\varepsilon}, c_{1}^{\varepsilon}, c_{2}^{\varepsilon}\right)$ with $c_{2}^{\varepsilon}-c_{2 D} \in\left\{\phi \in L^{2}\left(0, T ; H^{1}(\Omega)\right) ; \phi=0\right.$ on $\left.\Gamma_{1} \cup \Gamma_{2}\right\}$, satisfying for a.e. $t \in(0, T)$

$$
\begin{aligned}
& \int_{\Omega} \partial_{t t} \mathbf{u}^{\varepsilon}(t) \varphi d x+2 \int_{\Omega_{f}^{\varepsilon}} D\left(\partial_{t} \mathbf{u}^{\varepsilon}(t)\right): D(\varphi) d x+ \\
& \frac{1}{\varepsilon^{2}} \int_{\Omega_{s}^{\varepsilon}} A\left(\mathcal{F}\left(c_{1}^{\varepsilon}\right)\right) D\left(\mathbf{u}^{\varepsilon}(t)\right): D(\varphi)=\frac{1}{\varepsilon^{2}} \int_{\Gamma_{2}}\left(\mathcal{P}_{1}^{\varepsilon}, \mathcal{S}_{2}^{\varepsilon}, \mathcal{S}_{3}^{\varepsilon}\right) \varphi d S, \\
& \nabla \cdot \partial_{t} \mathbf{u}^{\varepsilon}=0, \quad \text { in } \Omega_{f}^{\varepsilon} \times(0, T), \\
& \mathbf{u}^{\varepsilon}(x, 0)=0, \quad \partial_{t} \mathbf{u}^{\varepsilon}(x, 0)=0, \quad \text { in } \Omega, \\
& \int_{\Omega_{s}^{\varepsilon}} \partial_{t} c_{1}^{\varepsilon} \psi d x+\int_{\Omega_{s}^{\varepsilon}} d_{1}\left(c_{2}^{\varepsilon}\right) \nabla c_{1}^{\varepsilon}(t) \nabla \psi d x=\int_{\Omega_{s}^{\varepsilon}} g_{1}\left(c_{1}^{\varepsilon}, c_{2}^{\varepsilon}\right) \psi d x, \\
& \int_{\Omega}\left\{\chi_{\Omega_{f}^{\varepsilon}}+\frac{1}{K} \chi_{\Omega_{s}^{\varepsilon}}\right\} \partial_{t} c_{2}^{\varepsilon} \zeta d x+\int_{\Omega} d_{2}\left\{\chi_{\Omega_{f}^{\varepsilon}}+\frac{1}{K} \chi_{\Omega_{s}^{\varepsilon}}\right\} \nabla c_{2}^{\varepsilon}(t) \nabla \zeta d x \\
& -\int_{\Omega_{f}^{\varepsilon}} \partial_{t} \mathbf{u}^{\varepsilon}(t) c_{2}^{\varepsilon}(t) \nabla \zeta d x=\int_{\Omega}\left\{g_{2}\left(c_{2}^{\varepsilon}\right) \chi_{\Omega_{f}^{\varepsilon}}+g_{3}\left(c_{1}^{\varepsilon}, c_{2}^{\varepsilon}\right) \chi_{\Omega_{s}^{\varepsilon}}\right\} \zeta d x, \\
& c_{1}^{\varepsilon}(0)=c_{10} \text { in } \Omega_{s}^{\varepsilon}, \quad c_{2}^{\varepsilon}(0)=c_{20} \text { in } \Omega,
\end{aligned}
$$

for all $\varphi \in V, \psi \in H^{1}\left(\Omega_{s}^{\varepsilon}\right)$, and $\zeta \in H^{1}(\Omega)$ with $\zeta=0$ on $\Gamma_{1} \cup \Gamma_{2}$. The space $\mathrm{V}$ is defined as follows:

$$
V=\left\{\varphi \in H^{1}(\Omega)^{3} ; \quad \nabla \cdot \varphi=0 \text { in } \Omega_{f}^{\varepsilon}, \quad \varphi=0 \text { on } \Gamma_{3}\right\} .
$$

\section{Assumptions on the data and the main result.}

3.1. Assumptions on the data. We assume that the components of the symmetric fourth order elasticity tensor $A$ belong to $C^{3}(\mathbb{R})$ as functions of $\mathcal{F}$, and that there exists $\lambda_{0} \in \mathbb{R}, \lambda_{0}>0$ such that

$$
\lambda_{0}\|M\|^{2} \leq A(\cdot) M M \leq \frac{1}{\lambda_{0}}\|M\|^{2},
$$

for all symmetric matrices $\mathrm{M}$, a.e. on $\mathbb{R}$. Further, we suppose that the normal stresses $\left(\mathcal{P}_{1}^{\varepsilon}, \mathcal{S}_{2}^{\varepsilon}, \mathcal{S}_{3}^{\varepsilon}\right)$ on the boundary $\Gamma_{2}$ have the following properties: $\mathcal{P}_{1}^{\varepsilon} \in C^{3}\left([0, T], H^{1}\left(\Gamma_{2}\right)\right)$, such that $\left\|\mathcal{P}_{1}^{\varepsilon}\right\|_{C^{3}\left([0, T], H^{1}\left(\Gamma_{2}\right)\right)}$ is uniformly bounded with respect to $\varepsilon$, and

$$
\mathcal{P}_{1}^{\varepsilon} \rightarrow \mathcal{P}^{0} \quad \text { strongly in } L^{2}\left((0, T) \times \Gamma_{2}\right),
$$

with $\mathcal{P}^{0} \in C^{3}\left([0, T], H^{5 / 2}\left(\Gamma_{2}\right)\right)$. Furthermore,

$$
\mathcal{S}_{j}^{\varepsilon}=\chi_{\bar{\Omega}_{s}^{\varepsilon} \cap \Gamma_{2}} \mathcal{C}_{j}^{s}+\varepsilon \chi_{\bar{\Omega}_{f}^{\varepsilon} \cap \Gamma_{2}} \mathcal{C}_{j}^{f},
$$

with

$$
\left\|\mathcal{C}_{j}^{s}\right\|_{C^{3}\left([0, T], H^{\frac{1}{2}}\left(\Gamma_{2}\right)\right)}+\left\|\mathcal{C}_{j}^{f}\right\|_{C^{3}\left([0, T], H^{\frac{1}{2}}\left(\Gamma_{2}\right)\right)} \leq C,
$$

for $j=2,3$. Finally, we suppose that

$$
\left(\mathcal{P}_{1}^{\varepsilon}, \mathcal{S}_{2}^{\varepsilon}, \mathcal{S}_{3}^{\varepsilon}\right)(0)=\partial_{t}\left(\mathcal{P}_{1}^{\varepsilon}, \mathcal{S}_{2}^{\varepsilon}, \mathcal{S}_{3}^{\varepsilon}\right)(0)=\partial_{t t}\left(\mathcal{P}_{1}^{\varepsilon}, \mathcal{S}_{2}^{\varepsilon}, \mathcal{S}_{3}^{\varepsilon}\right)(0)=0
$$

For the diffusion coefficients, we assume that $d_{1} \in C^{2}(\mathbb{R})$ is strictly positive, bounded and Lipschitz continuous and $d_{2}>0$. Concerning the reaction terms, we assume that 
$g_{1}, g_{2}, g_{3}$ are Lipschitz continuous with respect to their arguments. This implies that there exist positive constants $C_{1}, C_{2}$ and $C_{3}$ such that

$$
\begin{aligned}
\left|g_{1}(y, z)\right| & \leq C_{1}(1+|(y, z)|) \quad \text { for all }(y, z) \in \mathbb{R}^{2} \\
\left|g_{2}(z)\right| & \leq C_{2}(1+|z|) \quad \text { for all } z \in \mathbb{R} \\
\left|g_{3}(y, z)\right| & \leq C_{3}(1+|(y, z)|) \quad \text { for all }(y, z) \in \mathbb{R}^{2}
\end{aligned}
$$

Additionally we have to impose on $g_{1}, g_{2}, g_{3}$ structural conditions which guarantee positivity of the solutions and for $c_{1}^{\varepsilon}$ also a uniform upper bound. A possible choice of such conditions is given in the following.

$$
\begin{aligned}
& x^{-} g_{1}\left(x^{-}, y\right) \leq C\left(x^{-}\right)^{2} \\
& y^{-} g_{2}\left(y^{-}\right) \leq C\left(y^{-}\right)^{2} \\
& y^{-} g_{3}\left(x, y^{-}\right) \leq C\left(\left(x^{-}\right)^{2}+\left(y^{-}\right)^{2}\right)
\end{aligned}
$$

for all $x, y \in \mathbb{R}$, where $x^{-}=\min \{x, 0\}$. We also require that there exist constants $A_{1}, M_{1} \in \mathbb{R}, A_{1} \geq 0, M_{1}>0$, such that

$$
g_{1}(x, y) \leq A_{1} x, \quad \text { for } x \geq M_{1}, y \in \mathbb{R} .
$$

For the initial and boundary concentrations we assume that

$$
c_{10} \in C^{2}\left(\bar{\Omega}_{s}^{\varepsilon}\right) \text { with } \nabla c_{10} \cdot \nu=0 \text { on } \partial \Omega_{s}^{\varepsilon} \text {, and } 0 \leq c_{10} \leq M_{1},
$$

where $M_{1}$ is the constant in the assumption (3.12). We also assume that there exists $\beta>0$ and $M_{2}>0$, such that

$$
c_{20} \in H^{1}(\Omega) \cap C^{\beta}(\bar{\Omega}) \cap C^{2+\beta}\left(\bar{\Omega}_{s}^{\varepsilon}\right) \cap C^{2+\beta}\left(\bar{\Omega}_{f}^{\varepsilon}\right)
$$

and

$$
\left.c_{20}\right|_{\left(\Gamma_{1} \cup \Gamma_{2}\right)}=\left.c_{2 D}\right|_{\left(\Gamma_{1} \cup \Gamma_{2}\right) \times\{0\}}, \quad \nabla c_{20} \cdot \nu=0 \text { on } \Gamma_{3} \text {, and } 0 \leq c_{20} \leq M_{2} .
$$

Finally, for the boundary concentration $c_{2 D}$ we require

$$
\begin{aligned}
& c_{2 D} \in H^{1}(\Omega \times(0, T)) \cap C^{\beta, \frac{\beta}{2}}(\bar{\Omega} \times[0, T]), \\
& c_{2 D} \in C^{2+\beta, 1+\frac{\beta}{2}}\left(\bar{\Omega}_{s}^{\varepsilon} \times[0, T]\right) \cap C^{2+\beta, 1+\frac{\beta}{2}}\left(\bar{\Omega}_{f}^{\varepsilon} \times[0, T]\right),
\end{aligned}
$$

and

$$
0 \leq c_{2 D} \leq M_{2}
$$

3.2. Main result. In the limit $\varepsilon \rightarrow 0$, the solutions of the microscopic system (2.9)-(2.27) converge to the unique solution of the following homogenized system of differential equations. The effective system for the homogenized fluid-structure variables $\mathbf{u}^{0}, \mathbf{u}^{1}, p_{f}, \mathbf{w}^{0}, \pi^{0}$, consists of the homogenized equations for the structure 
variables:

$$
\begin{array}{r}
-\operatorname{Div}_{y}\left\{\mathcal{A}\left(\mathcal{F}\left(c_{1}^{0}\right)(t, x)\right)\left(D_{x}\left(\mathbf{u}^{0}\right)+D_{y}\left(\mathbf{u}^{1}\right)\right)\right\}=0 \text { in }(0, T) \times \Omega \times Y_{s} \\
\mathcal{A}\left(\mathcal{F}\left(c_{1}^{0}\right)(t, x)\right)\left(D_{x}\left(\mathbf{u}^{0}\right)+D_{y}\left(\mathbf{u}^{1}\right)\right) \cdot \nu+p_{f} \chi_{Y_{f}}(y) \cdot \nu=0 \\
\text { on }(0, T) \times \Omega \times\left(\partial Y_{s} \backslash \partial Y\right)
\end{array}
$$

$\mathbf{u}^{1}$ is $Y$ - periodic

$$
\begin{gathered}
-\operatorname{Div}_{x}\left\{\int_{Y_{s}} \mathcal{A}\left(\mathcal{F}\left(c_{1}^{0}\right)\right)\left(D_{x}\left(\mathbf{u}^{0}\right)+D_{y}\left(\mathbf{u}^{1}\right)\right) d y\right\}+\left|Y_{f}\right| \nabla_{x} p_{f}(t, x)=0 \\
\text { in }(0, T) \times \Omega
\end{gathered}
$$

$\mathbf{u}^{0}(t, x)=0 \quad$ on $(0, T) \times \Gamma_{3}$,

coupled with the generalized Darcy's law for the fluid variables:

$$
\begin{aligned}
& -\Delta_{y}\left(\partial_{t} \mathbf{w}^{0}\right)+\nabla_{y} \pi^{0}=-\nabla_{x} p_{f} \quad \text { in }(0, T) \times \Omega \times Y_{f} \\
& \operatorname{div}_{y}\left(\partial_{t} \mathbf{w}^{0}\right)=0 \quad \text { in }(0, T) \times \Omega \times Y_{f} \\
& \mathbf{w}^{0}=0 \quad \text { on }(0, T) \times \Omega \times \partial\left(Y_{f} \backslash \partial Y\right) \\
& \mathbf{w}^{0}, \pi^{0} \text { are } Y \text { - periodic } \\
& \operatorname{div}_{x}\left(\left|Y_{f}\right| \partial_{t} \mathbf{u}^{0}(t, x)+\int_{Y_{f}} \partial_{t} \mathbf{w}^{0}(t, x, y) d y\right)=\int_{Y_{s}} \operatorname{div}_{y} \partial_{t} \mathbf{u}^{1}(t, x, y) d y \\
& p_{f}=0 \text { on }(0, T) \times \Gamma_{1} \\
& p_{f}=\mathcal{P}^{0} \quad \text { on }(0, T) \times \Gamma_{2} \\
& \int_{Y_{f}} \partial_{t} \mathbf{w}^{0} d y \cdot \nu=0 \quad \text { on }(0, T) \times \Omega \\
&
\end{aligned}
$$

Here, the tensor $\mathcal{A}$ is given by

$$
\mathcal{A}\left(\mathcal{F}\left(c_{1}^{0}\right)\right)(x, t)=\mathcal{A}\left(\int_{0}^{t} \mathcal{K}(t-\tau) F\left(c_{1}^{0}(\tau, x)\right) d \tau\right) .
$$

The effective system for the fluid-structure-interaction is coupled with the homogenized system for the concentrations $c_{1}^{0}, c_{1}^{1}, c_{2}^{0}$, and $c_{2}^{1}$. The two-scale homogenized 
problem for the first chemical is given by:

$$
\begin{aligned}
& -\operatorname{div}_{y}\left\{d_{1}\left(c_{2}^{0}\right)\left(\nabla_{x} c_{1}^{0}(t, x)+\nabla_{y} c_{1}^{1}(t, x, y)\right)\right\}=0 \quad \text { in }(0, T) \times \Omega \times Y_{s} \\
& d_{1}\left(c_{2}^{0}\right)\left(\nabla_{x} c_{1}^{0}+\nabla_{y} c_{1}^{1}\right) \cdot \nu=0 \quad \text { on }(0, T) \times \Omega \times\left(\partial Y_{s} \backslash \partial Y\right) \\
& c_{1}^{1} \text { is } Y \text { - periodic } \\
& \left|Y_{s}\right| \partial_{t} c_{1}^{0}-\operatorname{div}_{x}\left(\int_{Y_{s}} d_{1}\left(c_{2}^{0}\right)\left(\nabla_{x} c_{1}^{0}(t, x)+\nabla_{y} c_{1}^{1}(t, x, y)\right) d y\right) \\
& =\left|Y_{s}\right| g\left(c_{1}^{0}, c_{2}^{0}\right) \quad \text { on }(0, T) \times \Omega \\
& \int_{Y_{s}} d_{1}\left(c_{2}^{0}\right)\left(\nabla_{x} c_{1}^{0}(t, x)+\nabla_{y} c_{1}^{1}(t, x, y)\right) d y \cdot \nu=0 \quad \text { on }(0, T) \times \partial \Omega \\
& c_{1}^{0}(0, x)=c_{10} \quad \text { in } \Omega
\end{aligned}
$$

The tow-scale homogenized problem for the concentration of the second chemical species reads:

$$
\begin{aligned}
& -\operatorname{div}_{y}\left\{k(y)\left(\nabla_{x} c_{2}^{0}(t, x)+\nabla_{y} c_{2}^{1}(t, x, y)\right)-\chi_{Y_{f}}(y) \partial_{t} \mathbf{u}^{0} c_{2}^{0}\right\}=0 \\
& \text { in }(0, T) \times \Omega \times Y \\
& c_{1}^{2} \text { is } Y \text {-periodic } \\
& \left(\int_{Y} k(y) d y\right) \partial_{t} c_{2}^{0}-d_{2} \operatorname{div}_{x}\left(\int_{Y} k(y)\left(\nabla_{x} c_{2}^{0}(t, x)+\nabla_{y} c_{2}^{1}(t, x, y)\right) d y\right) \\
& +\operatorname{div}_{x}\left(\left(\left|Y_{f}\right| \partial_{t} \mathbf{u}^{0}+\int_{Y_{f}} \partial_{t} \mathbf{w}^{0}\right) c_{2}^{0}\right) \\
& =\left|Y_{s}\right| g_{2}\left(c_{1}^{0}, c_{2}^{0}\right)+\left|Y_{f}\right| g_{3}\left(c_{2}^{0}\right) \quad \text { in }(0, T) \times \Omega \\
& \left(d_{2} \int_{Y} k(y)\left(\nabla_{x} c_{2}^{0}+\nabla_{y} c_{2}^{1}\right) d y-\left(\left|Y_{f}\right| \partial_{t} \mathbf{u}^{0}+\int_{Y_{f}} \partial_{t} \mathbf{w}^{0}\right) c_{2}^{0}\right) \cdot \nu=0 \\
& \text { on }(0, T) \times \Gamma_{3} \\
& c_{2}^{0}=c_{2 D} \quad \text { on }(0, T) \times\left(\Gamma_{1} \cup \Gamma_{2}\right) \\
& c_{2}^{0}(0, x)=c_{20} \text { in } \Omega \text {. }
\end{aligned}
$$

Here, we denoted

$$
k(y):=\chi_{Y_{f}}(y)+\frac{1}{K} \chi_{Y_{s}}(y) .
$$

Besides the standard convergences of the microscopic variables to the effective ones, given in Theorems 5.3, and Theorem 5.4, we also prove the following convergences of the energies:

TheOrem 3.1. For the limit functions $\mathbf{u}^{0}, \mathbf{u}^{1}, \mathbf{w}^{0}$, and $c_{1}^{0}$, we have the following convergences in energy:

$$
\begin{aligned}
& \lim _{\varepsilon \rightarrow 0} \int_{0}^{T} \int_{\Omega_{s}^{\varepsilon}} A\left(\mathcal{F}\left(c_{1}^{\varepsilon}\right)\right) D\left(\mathbf{u}^{\varepsilon}\right): D\left(\mathbf{u}^{\varepsilon}\right) d x d t \\
& =\int_{0}^{T} \int_{\Omega} \int_{Y_{s}} A\left(\mathcal{F}\left(c_{1}^{0}\right)\right)\left(D_{x}\left(\mathbf{u}^{\mathbf{0}}\right)+D_{y}\left(\mathbf{u}^{\mathbf{1}}\right)\right):\left(D_{x}\left(\mathbf{u}^{\mathbf{0}}\right)+D_{y}\left(\mathbf{u}^{\mathbf{1}}\right)\right) d y d x d t, \\
& \lim _{\varepsilon \rightarrow 0} \int_{\Omega_{f}^{\varepsilon}}\left|\varepsilon D\left(\mathbf{u}^{\varepsilon}\right)\right|^{2}(t) d x=\int_{\Omega} \int_{Y_{f}}\left|D_{y}\left(\mathbf{w}^{\mathbf{0}}\right)\right|^{2}(t) d x,
\end{aligned}
$$




$$
\begin{aligned}
& \lim _{\varepsilon \rightarrow 0} \int_{\Omega_{s}^{\varepsilon}} A\left(\mathcal{F}\left(c_{1}^{\varepsilon}(t)\right)\right) D\left(\mathbf{u}^{\varepsilon}(t)\right): D\left(\mathbf{u}^{\varepsilon}(t)\right) d x d t \\
& =\int_{\Omega} \int_{Y_{s}} A\left(\mathcal{F}\left(c_{1}^{0}(t)\right)\right)\left(D_{x}\left(\mathbf{u}^{\mathbf{0}}(t)\right)+D_{y}\left(\mathbf{u}^{\mathbf{1}}\right)\right):\left(D_{x}\left(\mathbf{u}^{\mathbf{0}}(t)\right)+D_{y}\left(\mathbf{u}^{\mathbf{1}}\right)(t)\right) d y d x, \\
& \text { a.e. on }(0, T), \\
& \lim _{\varepsilon \rightarrow 0} \int_{0}^{T} \int_{\Omega_{f}^{\varepsilon}} \varepsilon^{2}\left|D\left(\partial_{t} \mathbf{u}^{\varepsilon}\right)\right|^{2} d x d t=\int_{0}^{T} \int_{\Omega} \int_{Y_{f}}\left|D_{y}\left(\partial_{t} \mathbf{w}^{\mathbf{0}}\right)\right|^{2} d x d t .
\end{aligned}
$$

The convergence of the energies imply strong two-scale convergence results for the fluid-structure variables:

COROLlary 3.2. The following strong two-scale convergence results hold

$$
\lim _{\varepsilon \rightarrow 0} \int_{\Omega_{s}^{\varepsilon}}\left|D\left(\mathbf{u}^{\varepsilon}(t, x)-\mathbf{u}^{0}(t, x)-\varepsilon \mathbf{u}^{1}\left(t, x, \frac{x}{\varepsilon}\right)\right)\right|^{2} d x=0,
$$

for almost every $t \in(0, T)$, and

$$
\lim _{\varepsilon \rightarrow 0} \int_{0}^{T} \int_{\Omega_{f}^{\varepsilon}} \varepsilon^{2}\left|D\left(\partial_{t} \mathbf{u}^{\varepsilon}(t, x)-\partial_{t} \mathbf{w}^{0}\left(t, x, \frac{x}{\varepsilon}\right)\right)\right|^{2} d x d t=0 .
$$

4. Estimates of the microscopic solutions. In this section we prove estimates for the solutions of the microscopic problem (2.9)-(2.27). Herby, the dependence of the parameter $\varepsilon$ is given explicitly. Based on these estimates, we are able to perform the homogenization limit $\varepsilon \rightarrow 0$, which leads to a macroscopic description of the investigated processes.

A fundamental tool in the proof of estimates is given in the following.

Proposition 4.1. Let $\xi \in C\left([0, T], H^{1}(\Omega)\right)^{3}$ with

$$
\xi(0)=0, \text { and } \xi(t, x)=0, \text { for a.e. }(t, x) \in(0, T) \times \Gamma_{3} .
$$

The following estimate holds for all $t \in[0, T]$, with a constant $C$ independent of $\varepsilon$

$$
\|\xi(t)\|_{L^{2}(\Omega)^{3}} \leq C\left\{\|D(\xi(t))\|_{L^{2}\left(\Omega_{s}^{\varepsilon}\right)^{9}}+\varepsilon \int_{0}^{t} \| D\left(\partial_{\tau} \xi(\tau) \|_{L^{2}\left(\Omega_{f}^{\varepsilon}\right)^{9}} d \tau\right\} .\right.
$$

Proof. For every $t \in[0, T]$, let $\hat{\xi}(t)$ be the $H^{1}$-extension of $\left.\xi\right|_{\Omega_{s}^{\varepsilon}}$ to $\Omega$, as in [1]. Let $\omega(t)=\hat{\xi}(t)-\xi(t)$ on $\Omega_{f}^{\varepsilon}$. Then for every $t \in(0, T)$, we have $\omega(t) \in H^{1}\left(\Omega_{f}^{\varepsilon}\right)^{3}$, and $\omega(t, x)=0$ for $x \in \Gamma^{\varepsilon}$. Thus Poincare's inequality for rigid, periodic porous media together with Korn's inequality imply

$$
\|\omega(t)\|_{L^{2}\left(\Omega_{f}^{\varepsilon}\right)^{3}} \leq C \varepsilon\|\nabla \omega(t)\|_{L^{2}\left(\Omega_{f}^{\varepsilon}\right)^{9}} \leq C \varepsilon\|D(\omega(t))\|_{L^{2}\left(\Omega_{f}^{\varepsilon}\right)^{9}}
$$

for all $t \in(0, T)$. Here we use Korn's inequality on the $\varepsilon$-dependent domain $\Omega_{f}^{\varepsilon}$, see e.g. Theorem 4.5, Chapter 1 in [14]. Using (4.3), and the properties of the extension $\hat{\xi}$, see $[1]$, we obtain

$$
\begin{aligned}
& \|\xi(t)\|_{L^{2}\left(\Omega_{f}^{\varepsilon}\right)^{3}} \\
& \leq\|\hat{\xi}(t)\|_{L^{2}\left(\Omega_{f}^{\varepsilon}\right)^{3}}+C \varepsilon\left\{\|D(\hat{\xi}(t))\|_{L^{2}\left(\Omega_{f}^{\varepsilon}\right)^{9}}+\|D(\xi(t))\|_{L^{2}\left(\Omega_{f}^{\varepsilon}\right)^{9}}\right\} \\
& \leq C\|\xi(t)\|_{L^{2}\left(\Omega_{s}^{\varepsilon}\right)^{3}}+C\|D(\xi(t))\|_{L^{2}\left(\Omega_{s}^{\varepsilon}\right)^{9}}+C \varepsilon\|D(\xi(t))\|_{L^{2}\left(\Omega_{f}^{\varepsilon}\right)^{9}} \\
& \leq C\|D(\xi(t))\|_{L^{2}\left(\Omega_{s}^{\varepsilon}\right)^{9}}+C \varepsilon\|D(\xi(t))\|_{L^{2}\left(\Omega_{f}^{\varepsilon}\right)^{9}}
\end{aligned}
$$


The last estimate in (4.4) follows from the fact that the displacement is zero at the lateral boundary $\Gamma_{3}$, and we have a Poincare type inequality for the extended displacement. Next, we remark that using $\xi(0)=0$, we have

$$
\|D(\xi(t))\|_{L^{2}\left(\Omega_{f}^{\varepsilon}\right)^{9}} \leq\left\|\int_{0}^{t} D\left(\partial_{\tau} \xi(\tau)\right) d \tau\right\|_{L^{2}\left(\Omega_{f}^{\varepsilon}\right)^{9}} \leq \int_{0}^{t}\left\|D\left(\partial_{\tau} \xi(\tau)\right)\right\|_{L^{2}\left(\Omega_{f}^{\varepsilon}\right)^{9}} d \tau .
$$

Estimates (4.4), and (4.5) now imply (4.2), and the proposition is proved.

We first derive uniform $L^{\infty}$-estimates for the concentration $c_{1}^{\varepsilon}$. Since $c_{1}^{\varepsilon}$ enters the elasticity coefficients, these estimates are also needed when proving estimates for the fluid-structure variables.

Proposition 4.2. Under the assumptions on the data from Section 3, the following estimates hold

$$
0 \leq c_{1}^{\varepsilon} \leq M_{1} e^{A_{1} t} \quad \text { a.e. on }[0, T] \times \Omega_{s}^{\varepsilon},
$$

where the constants $A_{1}$, and $M_{1}$ are given by (3.12).

Proof. The proof of the estimates for $c_{1}^{\varepsilon}$ is based on the assumptions (3.9), and (3.12), for the nonlinear reaction term $g_{1}$, and is done analogously to the corresponding proof for positivity and boundedness of the concentration $c_{1}$ in [10].

Proposition 4.3. The solutions to problem (2.9)-(2.27) satisfy the following estimates, with a constant $C$ independent of $\varepsilon$

$$
\begin{aligned}
& \left\|\partial_{t} \mathbf{u}^{\varepsilon}\right\|_{L^{\infty}\left(0, T ; L^{2}(\Omega)\right)}+\left\|\partial_{t t} \mathbf{u}^{\varepsilon}\right\|_{L^{\infty}\left(0, T ; L^{2}(\Omega)\right)} \leq \frac{C}{\varepsilon} \\
& \left\|\partial_{t t t} \mathbf{u}^{\varepsilon}\right\|_{L^{\infty}\left(0, T ; L^{2}(\Omega)\right)}+\left\|\partial_{t t t t} \mathbf{u}^{\varepsilon}\right\|_{L^{\infty}\left(0, T ; L^{2}(\Omega)\right)} \leq \frac{C}{\varepsilon} \\
& \left\|D\left(\mathbf{u}^{\varepsilon}\right)\right\|_{L^{\infty}\left(0, T ; L^{2}\left(\Omega_{s}^{\varepsilon}\right)\right)}+\left\|D\left(\partial_{t} \mathbf{u}^{\varepsilon}\right)\right\|_{L^{\infty}\left(0, T ; L^{2}\left(\Omega_{s}^{\varepsilon}\right)\right)} \leq C \\
& \left\|D\left(\partial_{t t} \mathbf{u}^{\varepsilon}\right)\right\|_{L^{\infty}\left(0, T ; L^{2}\left(\Omega_{s}^{\varepsilon}\right)\right)}+\left\|D\left(\partial_{t t t} \mathbf{u}^{\varepsilon}\right)\right\|_{L^{\infty}\left(0, T ; L^{2}\left(\Omega_{s}^{\varepsilon}\right)\right)} \leq C \\
& \left\|D\left(\partial_{t} \mathbf{u}^{\varepsilon}\right)\right\|_{\left.L^{2}\left((0, T) \times \Omega_{f}^{\varepsilon}\right)\right)}+\left\|D\left(\partial_{t t} \mathbf{u}^{\varepsilon}\right)\right\|_{\left.L^{2}\left((0, T) \times \Omega_{f}^{\varepsilon}\right)\right)} \leq \frac{C}{\varepsilon} \\
& \left\|D\left(\partial_{t t t} \mathbf{u}^{\varepsilon}\right)\right\|_{\left.L^{2}\left((0, T) \times \Omega_{f}^{\varepsilon}\right)\right)}+\left\|D\left(\partial_{t t t t} \mathbf{u}^{\varepsilon}\right)\right\|_{\left.L^{2}\left((0, T) \times \Omega_{f}^{\varepsilon}\right)\right)} \leq \frac{C}{\varepsilon}
\end{aligned}
$$

Proof. We start from the variational formulation (2.28)-(2.33), and insert $\varphi=$ $\partial_{t} \mathbf{u}^{\varepsilon}$ as test function. It yields

$$
\begin{aligned}
& \int_{0}^{t} \int_{\Omega} \partial_{\tau \tau} \mathbf{u}^{\varepsilon} \partial_{\tau} \mathbf{u}^{\varepsilon}+2 \int_{0}^{t} \int_{\Omega_{f}^{\varepsilon}}\left|D\left(\partial_{\tau} \mathbf{u}^{\varepsilon}\right)\right|^{2}+ \\
& \frac{1}{\varepsilon^{2}} \int_{0}^{t} \int_{\Omega_{s}^{\varepsilon}} A\left(\mathcal{F}\left(c_{1}^{\varepsilon}\right)\right) D\left(\mathbf{u}^{\varepsilon}\right): D\left(\partial_{\tau} \mathbf{u}^{\varepsilon}\right)=\frac{1}{\varepsilon^{2}} \int_{0}^{t} \int_{\Gamma_{2}}\left(\mathcal{P}_{1}^{\varepsilon}, \mathcal{S}_{2}^{\varepsilon}, \mathcal{S}_{3}^{\varepsilon}\right) \partial_{\tau} \mathbf{u}^{\varepsilon} d S .
\end{aligned}
$$

Let us first transform the third term as follows

$$
\begin{aligned}
& \int_{0}^{t} \int_{\Omega_{s}^{\varepsilon}} A\left(\mathcal{F}\left(c_{1}^{\varepsilon}\right)\right) D\left(\mathbf{u}^{\varepsilon}\right): D\left(\partial_{\tau} \mathbf{u}^{\varepsilon}\right) \\
& =\int_{\Omega_{s}^{\varepsilon}} A\left(\mathcal{F}\left(c_{1}^{\varepsilon}(t)\right)\right) D\left(\mathbf{u}^{\varepsilon}(t)\right): D\left(\mathbf{u}^{\varepsilon}(t)\right)-\int_{0}^{t} \int_{\Omega_{s}^{\varepsilon}} A\left(\mathcal{F}\left(c_{1}^{\varepsilon}\right)\right) D\left(\partial_{\tau} \mathbf{u}^{\varepsilon}\right): D\left(\mathbf{u}^{\varepsilon}\right) \\
& -\int_{0}^{t} \int_{\Omega_{s}^{\varepsilon}} \frac{d A}{d \mathcal{F}}\left(\mathcal{F}\left(c_{1}^{\varepsilon}\right)\right) \frac{d \mathcal{F}}{d t}\left(c_{1}^{\varepsilon}\right) D\left(\mathbf{u}^{\varepsilon}\right): D\left(\mathbf{u}^{\varepsilon}\right) \frac{d \mathcal{F}}{d t}\left(c_{1}^{\varepsilon}\right)
\end{aligned}
$$


Thus, we have

$$
\begin{aligned}
& \int_{0}^{t} \int_{\Omega_{s}^{\varepsilon}} A\left(\mathcal{F}\left(c_{1}^{\varepsilon}\right)\right) D\left(\mathbf{u}^{\varepsilon}\right): D\left(\partial_{\tau} \mathbf{u}^{\varepsilon}\right) \\
& =\frac{1}{2} \int_{\Omega_{s}^{\varepsilon}} A\left(\mathcal{F}\left(c_{1}^{\varepsilon}(t)\right)\right) D\left(\mathbf{u}^{\varepsilon}(t)\right): D\left(\mathbf{u}^{\varepsilon}(t)\right) \\
& -\frac{1}{2} \int_{0}^{t} \int_{\Omega_{s}^{\varepsilon}} \frac{d A}{d \mathcal{F}}\left(\mathcal{F}\left(c_{1}^{\varepsilon}\right)\right) \frac{d \mathcal{F}}{d t}\left(c_{1}^{\varepsilon}\right) D\left(\mathbf{u}^{\varepsilon}\right): D\left(\mathbf{u}^{\varepsilon}\right)
\end{aligned}
$$

We start by estimating the boundary term in (4.13). To this end, we introduce $\tilde{\mathcal{P}}_{1}^{\varepsilon}$ by

$$
\tilde{\mathcal{P}}_{1}^{\varepsilon}\left(x_{1}, x_{2}, x_{3}, t\right)=\mathcal{P}_{1}^{\varepsilon}\left(x_{2}, x_{3}, t\right) x_{1} .
$$

Obviously, $\left.\tilde{\mathcal{P}}_{1}^{\varepsilon}\right|_{\Gamma_{2}}=\mathcal{P}_{1}^{\varepsilon}$, and we have

$$
\begin{aligned}
& \int_{0}^{t} \int_{\Gamma_{2}}\left(\mathcal{P}_{1}^{\varepsilon}, 0,0\right) \partial_{\tau} \mathbf{u}^{\varepsilon} d S=\int_{0}^{t} \int_{\Gamma_{2}} \mathcal{P}_{1}^{\varepsilon} \partial_{\tau} \mathbf{u}^{\varepsilon}{ }_{1} d S \\
& =\int_{\Gamma_{2}} \mathcal{P}_{1}^{\varepsilon} \mathbf{u}^{\varepsilon}{ }_{1}(t) d S-\int_{0}^{t} \int_{\Gamma_{2}} \partial_{\tau} \mathcal{P}_{1}^{\varepsilon} \mathbf{u}^{\varepsilon}{ }_{1} d S d \tau .
\end{aligned}
$$

We estimate the first term on the right hand side of (4.15) as follows:

$$
\begin{aligned}
& \int_{\Gamma_{2}} \mathcal{P}_{1}^{\varepsilon} \mathbf{u}^{\varepsilon}{ }_{1}(t) d S=\int_{\partial \Omega} \mathcal{P}^{\varepsilon} \mathbf{u}^{\varepsilon}(t) \cdot \nu d S=\int_{\Omega} \operatorname{div}\left(\tilde{\mathcal{P}}_{1}^{\varepsilon} \mathbf{u}^{\varepsilon}(t)\right) d x \\
& =\int_{\Omega} \nabla \tilde{\mathcal{P}}_{1}^{\varepsilon} \mathbf{u}^{\varepsilon}(t) d x+\int_{\Omega_{s}^{\varepsilon}} \tilde{\mathcal{P}}_{1}^{\varepsilon} \operatorname{div} \mathbf{u}^{\varepsilon}(t) d x
\end{aligned}
$$

Using the assumptions on the data, and Proposition 4.1, we conclude that

$$
\begin{aligned}
& \left|\int_{\Gamma_{2}} \mathcal{P}_{1}^{\varepsilon} \mathbf{u}^{\varepsilon}{ }_{1}(t) d S\right| \leq C\left\|\mathbf{u}^{\varepsilon}(t)\right\|_{L^{2}(\Omega)^{3}}+C\left\|D\left(\mathbf{u}^{\varepsilon}(t)\right)\right\|_{L^{2}\left(\Omega_{s}^{\varepsilon}\right)^{9}} \\
& \leq C\left\{\left\|D\left(\mathbf{u}^{\varepsilon}(t)\right)\right\|_{L^{2}\left(\Omega_{s}^{\varepsilon}\right)^{9}}+\varepsilon \int_{0}^{t}\left\|D\left(\partial_{\tau} \mathbf{u}^{\varepsilon}(\tau)\right)\right\|_{L^{2}\left(\Omega_{f}^{\varepsilon}\right)^{9}} d \tau\right\}
\end{aligned}
$$

Next, the second term on the right hand side of (4.15) is estimated as follows:

$$
\begin{aligned}
& \left|\int_{0}^{t} \int_{\Gamma_{2}} \partial_{\tau} \mathcal{P}_{1}^{\varepsilon} \mathbf{u}^{\varepsilon}{ }_{1} d S d \tau\right| \\
& \leq\left|\int_{0}^{t} \int_{\Omega} \nabla\left(\partial_{\tau} \tilde{\mathcal{P}}_{1}^{\varepsilon}\right) \mathbf{u}^{\varepsilon} d x d \tau\right|+\left|\int_{0}^{t} \int_{\Omega_{s}^{\varepsilon}} \partial_{\tau} \tilde{\mathcal{P}}_{1}^{\varepsilon} \operatorname{div} \mathbf{u}^{\varepsilon} d x d \tau\right| \\
& \leq C \int_{0}^{t}\left\{\left\|D\left(\mathbf{u}^{\varepsilon}\right)\right\|_{L^{2}\left(\Omega_{s}^{\varepsilon}\right)^{9}}+\varepsilon\left\|D\left(\partial_{\tau} \mathbf{u}^{\varepsilon}\right)\right\|_{L^{2}\left(\Omega_{f}^{\varepsilon}\right)^{9}}\right\}
\end{aligned}
$$

To complete the estimate of the boundary term in (4.13), we still have to estimate following term:

$$
\begin{aligned}
& \int_{0}^{t} \int_{\Gamma_{2}}\left(0, \mathcal{S}_{2}^{\varepsilon}, \mathcal{S}_{3}^{\varepsilon}\right) \partial_{\tau} \mathbf{u}^{\varepsilon} d S \\
& =\int_{0}^{t} \int_{\Gamma_{2} \cap \overline{\Omega_{s}^{\varepsilon}}}\left(0, \mathcal{S}_{2}^{\varepsilon}, \mathcal{S}_{3}^{\varepsilon}\right) \partial_{\tau} \mathbf{u}^{\varepsilon} d S+\int_{0}^{t} \int_{\Gamma_{2} \cap \overline{\Omega_{f}^{\varepsilon}}}\left(0, \mathcal{S}_{2}^{\varepsilon}, \mathcal{S}_{3}^{\varepsilon}\right) \partial_{\tau} \mathbf{u}^{\varepsilon} d S \\
& =\int_{0}^{t} \int_{\Gamma_{2} \cap \overline{\Omega_{s}^{\varepsilon}}}\left(0, \mathcal{C}_{2}^{s}, \mathcal{C}_{3}^{s}\right) \partial_{\tau} \mathbf{u}^{\varepsilon} d S+\varepsilon \int_{0}^{t} \int_{\Gamma_{2} \cap \overline{\Omega_{f}^{\varepsilon}}}\left(0, \mathcal{C}_{2}^{f}, \mathcal{C}_{3}^{f}\right) \partial_{\tau} \mathbf{u}^{\varepsilon} d S
\end{aligned}
$$


where for the last equality, we have used the representation formula (3.3). Let us first consider the first term in the right hand side of (4.19).

$$
\begin{aligned}
& \int_{0}^{t} \int_{\Gamma_{2} \cap \overline{\Omega_{s}^{\varepsilon}}}\left(0, \mathcal{C}_{2}^{s}, \mathcal{C}_{3}^{s}\right) \partial_{\tau} \mathbf{u}^{\varepsilon} d S \\
& =\int_{\Gamma_{2} \cap \overline{\Omega_{s}^{\varepsilon}}}\left(0, \mathcal{C}_{2}^{s}, \mathcal{C}_{3}^{s}\right) \mathbf{u}^{\varepsilon}(t) d S-\int_{0}^{t} \int_{\Gamma_{2} \cap \overline{\Omega_{s}^{\varepsilon}}}\left(0, \partial_{\tau} \mathcal{C}_{2}^{s}, \partial_{\tau} \mathcal{C}_{3}^{s}\right) \mathbf{u}^{\varepsilon} d S d \tau \\
& =\int_{\Gamma_{2}}\left(0, \mathcal{C}_{2}^{s}, \mathcal{C}_{3}^{s}\right) \hat{\mathbf{u}}^{\varepsilon}(t) \chi_{\Gamma_{2} \cap \overline{\Omega_{s}^{\varepsilon}}} d S-\int_{0}^{t} \int_{\Gamma_{2}}\left(0, \partial_{\tau} \mathcal{C}_{2}^{s}, \partial_{\tau} \mathcal{C}_{3}^{s}\right) \chi_{\Gamma_{2} \cap \overline{\Omega_{s}^{\varepsilon}}} \hat{\mathbf{u}}^{\varepsilon} d S d \tau \\
& \leq C\left\{\left\|\left(0, \mathcal{C}_{2}^{s}, \mathcal{C}_{3}^{s}\right)\right\|_{L^{\infty}\left(0, T ; L^{2}\left(\Gamma_{2} \cap \overline{\Omega_{s}^{\varepsilon}}\right)\right)}\left\|\mathbf{u}^{\varepsilon}(t)\right\|_{H^{1}\left(\Omega_{s}^{\varepsilon}\right)}\right. \\
& \left.+\left\|\left(0, \mathcal{C}_{2}^{s}, \mathcal{C}_{3}^{s}\right)\right\|_{H^{1, \infty}\left(0, T ; L^{2}\left(\Gamma_{2} \cap \overline{\Omega_{s}^{\varepsilon}}\right)\right)} \int_{0}^{t}\left\|\mathbf{u}^{\varepsilon}(\tau)\right\|_{\left.H^{1}\left(\Omega_{s}^{\varepsilon}\right)\right)} d \tau\right\}
\end{aligned}
$$

where, for every $t \in[0, T], \hat{\mathbf{u}}^{\varepsilon}(t)$ is the $H^{1}$-extension of $\left.\mathbf{u}^{\varepsilon}\right|_{\Omega_{s}^{\varepsilon}}$ to $\Omega$, as in [1]. In a similar way, we estimate the second term in the right hand side of (4.19), and get

$$
\begin{aligned}
& \varepsilon \int_{0}^{t} \int_{\Gamma_{2} \cap \overline{\Omega_{f}^{\varepsilon}}}\left(0, \mathcal{C}_{2}^{f}, \mathcal{C}_{3}^{f}\right) \partial_{\tau} \mathbf{u}^{\varepsilon} d S \\
& \leq C \varepsilon\left\{\left\|\left(0, \mathcal{C}_{2}^{f}, \mathcal{C}_{3}^{f}\right)\right\|_{L^{\infty}\left(0, T ; L^{2}\left(\Gamma_{2} \cap \overline{\Omega_{f}^{\varepsilon}}\right)\right)}\left\|\mathbf{u}^{\varepsilon}(t)\right\|_{H^{1}\left(\Omega_{f}^{\varepsilon}\right)}\right. \\
& \left.+\left\|\left(0, \mathcal{C}_{2}^{f}, \mathcal{C}_{3}^{f}\right)\right\|_{H^{1, \infty}\left(0, T ; L^{2}\left(\Gamma_{2} \cap \overline{\Omega_{f}^{\varepsilon}}\right)\right)} \int_{0}^{t}\left\|\mathbf{u}^{\varepsilon}(\tau)\right\|_{\left.H^{1}\left(\Omega_{f}^{\varepsilon}\right)\right)} d \tau\right\} .
\end{aligned}
$$

Using now the results from (4.14) - (4.21) in (4.13), we obtain

$\frac{1}{2} \int_{0}^{t} \int_{\Omega} \partial_{\tau}\left(\partial_{\tau} \mathbf{u}^{\varepsilon}\right)^{2} d x d \tau+2 \int_{0}^{t} \int_{\Omega_{f}^{\varepsilon}}\left|D\left(\partial_{\tau} \mathbf{u}^{\varepsilon}\right)\right|^{2} d x d \tau+$

$\frac{1}{2 \varepsilon^{2}} \int_{\Omega_{s}^{\varepsilon}} A\left(\mathcal{F}\left(c_{1}^{\varepsilon}(t)\right)\right) D\left(\mathbf{u}^{\varepsilon}(t)\right): D\left(\mathbf{u}^{\varepsilon}(t)\right) d x$

$\leq \frac{1}{2 \varepsilon^{2}}\left|\int_{0}^{t} \int_{\Omega_{s}^{\varepsilon}} \frac{d A}{d \mathcal{F}}\left(\mathcal{F}\left(c_{1}^{\varepsilon}\right)\right) \frac{d \mathcal{F}}{d t}\left(c_{1}^{\varepsilon}\right) D\left(\mathbf{u}^{\varepsilon}\right): D\left(\mathbf{u}^{\varepsilon}\right)\right|$

$+\frac{C}{\varepsilon^{2}}\left\{\left\|D\left(\mathbf{u}^{\varepsilon}(t)\right)\right\|_{L^{2}\left(\Omega_{s}^{\varepsilon}\right)^{9}}+\int_{0}^{t}\left\|D\left(\mathbf{u}^{\varepsilon}(\tau)\right)\right\|_{L^{2}\left(\Omega_{s}^{\varepsilon}\right)^{9}} d \tau+\varepsilon \int_{0}^{t}\left\|D\left(\partial_{\tau} \mathbf{u}^{\varepsilon}(\tau)\right)\right\|_{L^{2}\left(\Omega_{f}^{\varepsilon}\right)^{9}} d \tau\right\}$

$\leq \frac{1}{2 \varepsilon^{2}}\left\|\frac{d A}{d \mathcal{F}}\right\|_{L^{\infty}}\left\|\frac{d \mathcal{F}}{d t}\right\|_{L^{\infty}}\left\|A^{-1}\right\|_{L^{\infty}} \int_{0}^{t} \int_{\Omega_{s}^{\varepsilon}} A\left(\mathcal{F}\left(c_{1}^{\varepsilon}\right)\right) D\left(\mathbf{u}^{\varepsilon}\right): D\left(\mathbf{u}^{\varepsilon}\right)$

$+\frac{C}{\varepsilon^{2}} \sqrt{\int_{\Omega_{s}^{\varepsilon}} A\left(\mathcal{F}\left(c_{1}^{\varepsilon}\right)\right) D\left(\mathbf{u}^{\varepsilon}\right): D\left(\mathbf{u}^{\varepsilon}\right)(t)}+\frac{C}{\varepsilon^{2}} \int_{0}^{t} \sqrt{\int_{\Omega_{s}^{\varepsilon}} A\left(\mathcal{F}\left(c_{1}^{\varepsilon}\right)\right) D\left(\mathbf{u}^{\varepsilon}\right): D\left(\mathbf{u}^{\varepsilon}\right)(\tau) d \tau}$

$+\frac{C}{\varepsilon} \int_{0}^{t}\left\|D\left(\partial_{\tau} \mathbf{u}^{\varepsilon}(\tau)\right)\right\|_{L^{2}\left(\Omega_{f}^{\varepsilon}\right)^{9}} d \tau$

Now, using the regularity properties of the elasticity coefficients, the uniform bounds 
on $c_{1}^{\varepsilon}$ from Proposition 4.2, together with Gronwall's inequality, we obtain

$$
\begin{aligned}
& \int_{\Omega}\left|\partial_{t} \mathbf{u}^{\varepsilon}(t)\right|^{2} d x+2 \int_{0}^{t} \int_{\Omega_{f}^{\varepsilon}}\left|D\left(\partial_{\tau} \mathbf{u}^{\varepsilon}\right)\right|^{2} d x d \tau \\
& +\frac{1}{2 \varepsilon^{2}} \int_{\Omega_{s}^{\varepsilon}} A\left(\mathcal{F}\left(c_{1}^{\varepsilon}(t)\right)\right) D\left(\mathbf{u}^{\varepsilon}(t)\right): D\left(\mathbf{u}^{\varepsilon}(t)\right) d x \leq \frac{C}{\varepsilon^{2}},
\end{aligned}
$$

and thus we arrive at

$$
\begin{aligned}
& \left\|\partial_{t} \mathbf{u}^{\varepsilon}\right\|_{L^{\infty}\left(0, T ; L^{2}(\Omega)\right)}+\left\|D\left(\partial_{t} \mathbf{u}^{\varepsilon}\right)\right\|_{\left.L^{2}\left((0, T) \times \Omega_{f}^{\varepsilon}\right)\right)} \leq \frac{C}{\varepsilon}, \\
& \left\|D\left(\mathbf{u}^{\varepsilon}\right)\right\|_{L^{\infty}\left(0, T ; L^{2}\left(\Omega_{s}^{\varepsilon}\right)\right)} \leq C .
\end{aligned}
$$

Next, we differentiate (2.28) with respect to t. It yields

$$
\begin{aligned}
& \int_{\Omega} \partial_{t t t} \mathbf{u}^{\varepsilon}(t) \varphi d x+2 \int_{\Omega_{f}^{\varepsilon}} D\left(\partial_{t t} \mathbf{u}^{\varepsilon}(t)\right): D(\varphi) d x \\
& +\frac{1}{\varepsilon^{2}} \int_{\Omega_{s}^{\varepsilon}} \frac{d A}{d \mathcal{F}}\left(\mathcal{F}\left(c_{1}^{\varepsilon}(t)\right)\right) \frac{d \mathcal{F}}{d t}\left(c_{1}^{\varepsilon}(t)\right) D\left(\mathbf{u}^{\varepsilon}(t)\right): D(\varphi) \\
& +\frac{1}{\varepsilon^{2}} \int_{\Omega_{s}^{\varepsilon}} A\left(\mathcal{F}\left(c_{1}^{\varepsilon}(t)\right)\right) D\left(\partial_{t} \mathbf{u}^{\varepsilon}(t)\right): D(\varphi)=\frac{1}{\varepsilon^{2}} \int_{\Gamma_{2}} \partial_{t}\left(\mathcal{P}_{1}^{\varepsilon}, \mathcal{S}_{2}^{\varepsilon}, \mathcal{S}_{3}^{\varepsilon}\right) \varphi d S .
\end{aligned}
$$

We test (4.23) by $\varphi=\partial_{t t} \mathbf{u}^{\varepsilon}$, and get

$$
\begin{aligned}
& \frac{1}{2} \int_{\Omega}\left|\partial_{t t} \mathbf{u}^{\varepsilon}(t)\right|^{2}-\frac{1}{2} \int_{\Omega}\left|\partial_{t t} \mathbf{u}^{\varepsilon}(0)\right|^{2}+2 \int_{0}^{t} \int_{\Omega_{f}^{\varepsilon}}\left|D\left(\partial_{\tau \tau} \mathbf{u}^{\varepsilon}\right)\right|^{2} \\
& +\frac{1}{\varepsilon^{2}} \int_{0}^{t} \int_{\Omega_{s}^{\varepsilon}} \frac{d A}{d \mathcal{F}}\left(\mathcal{F}\left(c_{1}^{\varepsilon}\right)\right) \frac{d \mathcal{F}}{d \tau}\left(c_{1}^{\varepsilon}\right) D\left(\mathbf{u}^{\varepsilon}\right): D\left(\partial_{\tau \tau} \mathbf{u}^{\varepsilon}\right) \\
& +\frac{1}{\varepsilon^{2}} \int_{0}^{t} \int_{\Omega_{s}^{\varepsilon}} A\left(\mathcal{F}\left(c_{1}^{\varepsilon}\right)\right) D\left(\partial_{\tau} \mathbf{u}^{\varepsilon}\right): D\left(\partial_{\tau \tau} \mathbf{u}^{\varepsilon}\right) \\
& =\frac{1}{\varepsilon^{2}} \int_{0}^{t} \int_{\Gamma_{2}} \partial_{\tau}\left(\mathcal{P}_{1}^{\varepsilon}, \mathcal{S}_{2}^{\varepsilon}, \mathcal{S}_{3}^{\varepsilon}\right) \partial_{\tau \tau} \mathbf{u}^{\varepsilon} d S d \tau
\end{aligned}
$$

To get an information about $\partial_{t t} \mathbf{u}^{\varepsilon}(0)$, we evaluate equation (2.28) at $t=0$, and obtain for all $\varphi \in V$

$$
\begin{aligned}
& \int_{\Omega} \partial_{t t} \mathbf{u}^{\varepsilon}(0) \varphi d x+2 \int_{\Omega_{f}^{\varepsilon}} D\left(\partial_{t} \mathbf{u}^{\varepsilon}(0)\right): D(\varphi) d x+ \\
& \frac{1}{\varepsilon^{2}} \int_{\Omega_{s}^{\varepsilon}} A\left(\mathcal{F}\left(c_{1}^{\varepsilon}(0)\right)\right) D\left(\mathbf{u}^{\varepsilon}(0)\right): D(\varphi)=\frac{1}{\varepsilon^{2}} \int_{\Gamma_{2}}\left(\mathcal{P}_{1}^{\varepsilon}, \mathcal{S}_{2}^{\varepsilon}, \mathcal{S}_{3}^{\varepsilon}\right)(0) \varphi d S .
\end{aligned}
$$

Now, the homogeneous initial conditions for $\mathbf{u}^{\varepsilon}$, and $\left(\mathcal{P}_{1}^{\varepsilon}, \mathcal{S}_{2}^{\varepsilon}, \mathcal{S}_{3}^{\varepsilon}\right)$, see (2.30), and (3.5), imply

$$
\int_{\Omega} \partial_{t t} \mathbf{u}^{\varepsilon}(0) \varphi d x=0, \quad \text { for all } \varphi \in V
$$

Thus, we have $\partial_{t t} \mathbf{u}^{\varepsilon}(0)=0$ for a. e. $x \in \Omega$. 
Next, we have to transform several terms in (4.23).

$$
\begin{aligned}
& \int_{0}^{t} \int_{\Omega_{s}^{\varepsilon}} A\left(\mathcal{F}\left(c_{1}^{\varepsilon}\right)\right) D\left(\partial_{\tau} \mathbf{u}^{\varepsilon}\right): D\left(\partial_{\tau \tau} \mathbf{u}^{\varepsilon}\right) \\
& =\frac{1}{2} \int_{\Omega_{s}^{\varepsilon}} A\left(\mathcal{F}\left(c_{1}^{\varepsilon}(t)\right)\right) D\left(\partial_{t} \mathbf{u}^{\varepsilon}(t)\right): D\left(\partial_{t} \mathbf{u}^{\varepsilon}(t)\right) \\
& -\frac{1}{2} \int_{0}^{t} \int_{\Omega_{s}^{\varepsilon}} \frac{d A}{d \mathcal{F}}\left(\mathcal{F}\left(c_{1}^{\varepsilon}\right)\right) \frac{d \mathcal{F}}{d \tau}\left(c_{1}^{\varepsilon}\right) D\left(\partial_{\tau} \mathbf{u}^{\varepsilon}\right): D\left(\partial_{\tau} \mathbf{u}^{\varepsilon}\right) \\
& \int_{0}^{t} \int_{\Omega_{s}^{\varepsilon}} \frac{d A}{d \mathcal{F}}\left(\mathcal{F}\left(c_{1}^{\varepsilon}\right)\right) \frac{d \mathcal{F}}{d \tau}\left(c_{1}^{\varepsilon}\right) D\left(\mathbf{u}^{\varepsilon}\right): D\left(\partial_{\tau \tau} \mathbf{u}^{\varepsilon}\right) \\
& =\int_{\Omega_{s}^{\varepsilon}} \frac{d A}{d \mathcal{F}}\left(\mathcal{F}\left(c_{1}^{\varepsilon}(t)\right)\right) \frac{d \mathcal{F}}{d t}\left(c_{1}^{\varepsilon}(t)\right) D\left(\mathbf{u}^{\varepsilon}(t)\right): D\left(\partial_{t} \mathbf{u}^{\varepsilon}(t)\right) \\
& -\int_{0}^{t} \int_{\Omega_{s}^{\varepsilon}} \frac{d A}{d \mathcal{F}}\left(\mathcal{F}\left(c_{1}^{\varepsilon}\right)\right) \frac{d \mathcal{F}}{d \tau}\left(c_{1}^{\varepsilon}\right) D\left(\partial_{\tau} \mathbf{u}^{\varepsilon}\right): D\left(\partial_{\tau} \mathbf{u}^{\varepsilon}\right) \\
& \int_{0}^{t} \int_{\Omega_{s}^{\varepsilon}}\left\{\frac{d^{2} A}{d \mathcal{F}^{2}}\left(\frac{d \mathcal{F}}{d \tau}\right)^{2}+\frac{d A}{d \mathcal{F}} \frac{d^{2} \mathcal{F}}{d \tau^{2}}\right\} D\left(\mathbf{u}^{\varepsilon}\right): D\left(\partial_{\tau} \mathbf{u}^{\varepsilon}\right)
\end{aligned}
$$

To estimate the right hand side in (4.23), we start with

$$
\int_{0}^{t} \int_{\Gamma_{2}} \partial_{\tau} \mathcal{P}_{1}^{\varepsilon} \partial_{\tau \tau} \mathbf{u}_{1}^{\varepsilon} d S d \tau=\int_{\Gamma_{2}} \partial_{t} \mathcal{P}_{1}^{\varepsilon} \partial_{t} \mathbf{u}_{1}^{\varepsilon}(t) d S-\int_{0}^{t} \int_{\Gamma_{2}} \partial_{\tau \tau} \mathcal{P}_{1}^{\varepsilon} \partial_{\tau} \mathbf{u}_{1}^{\varepsilon} d S d \tau
$$

leading to

$$
\begin{aligned}
& \left|\int_{0}^{t} \int_{\Gamma_{2}} \partial_{\tau} \mathcal{P}_{1}^{\varepsilon} \partial_{\tau \tau} \mathbf{u}_{1}^{\varepsilon}\right| \leq C\left\{\left\|\partial_{t} \mathbf{u}^{\varepsilon}(t)\right\|_{L^{2}(\Omega)^{3}}+\left\|D\left(\partial_{t} \mathbf{u}^{\varepsilon}(t)\right)\right\|_{L^{2}\left(\Omega_{s}^{\varepsilon}\right)^{9}}\right\} \\
& +C \int_{0}^{t}\left\{\left\|D\left(\partial_{\tau} \mathbf{u}^{\varepsilon}\right)\right\|_{L^{2}\left(\Omega_{s}^{\varepsilon}\right)^{9}}+\varepsilon\left\|D\left(\partial_{\tau \tau} \mathbf{u}^{\varepsilon}\right)\right\|_{L^{2}\left(\Omega_{f}^{\varepsilon}\right)^{9}}\right\} .
\end{aligned}
$$

Similarly, we have

$$
\begin{aligned}
& \left|\int_{0}^{t} \int_{\Gamma_{2} \cap \overline{\Omega_{s}^{\varepsilon}}} \partial_{\tau}\left(0, \mathcal{C}_{2}^{s}, \mathcal{C}_{3}^{s}\right) \partial_{\tau}^{2} \mathbf{u}^{\varepsilon} d S d \tau\right| \\
& \leq C\left\{\left\|\partial_{t} \mathbf{u}^{\varepsilon}(t)\right\|_{H^{1}\left(\Omega_{s}^{\varepsilon}\right)^{3}}+\int_{0}^{t}\left\|\partial_{\tau} \mathbf{u}^{\varepsilon}\right\|_{H^{1}\left(\Omega_{s}^{\varepsilon}\right)^{3}} d \tau\right\},
\end{aligned}
$$

and

$$
\begin{aligned}
& \left|\varepsilon \int_{0}^{t} \int_{\Gamma_{2} \cap \overline{\Omega_{f}^{\varepsilon}}} \partial_{\tau}\left(0, \mathcal{C}_{2}^{f}, \mathcal{C}_{3}^{f}\right) \partial_{\tau}^{2} \mathbf{u}^{\varepsilon} d S d \tau\right| \\
& \leq C \varepsilon\left\{\left\|\partial_{t} \mathbf{u}^{\varepsilon}(t)\right\|_{H^{1}\left(\Omega_{f}^{\varepsilon}\right)^{3}}+\int_{0}^{t}\left\|\partial_{\tau} \mathbf{u}^{\varepsilon}\right\|_{H^{1}\left(\Omega_{f}^{\varepsilon}\right)^{3}} d \tau\right\} . \\
& \leq C \varepsilon \int_{0}^{t}\left\|\partial_{\tau}^{2} \mathbf{u}^{\varepsilon}\right\|_{H^{1}\left(\Omega_{f}^{\varepsilon}\right)^{3}} d \tau .
\end{aligned}
$$


Inserting the results from (4.26) - (4.31) in (4.23), we obtain

$$
\begin{aligned}
& \frac{1}{2} \int_{\Omega}\left|\partial_{t t} \mathbf{u}^{\varepsilon}(t)\right|^{2}+2 \int_{0}^{t} \int_{\Omega_{f}^{\varepsilon}}\left|D\left(\partial_{\tau \tau} \mathbf{u}^{\varepsilon}\right)\right|^{2} \\
& +\frac{1}{2 \varepsilon^{2}} \int_{\Omega_{s}^{\varepsilon}} A\left(\mathcal{F}\left(c_{1}^{\varepsilon}(t)\right)\right) D\left(\partial_{t} \mathbf{u}^{\varepsilon}(t)\right): D\left(\partial_{t} \mathbf{u}^{\varepsilon}(t)\right) d x \\
& \leq \frac{1}{\varepsilon^{2}} \int_{0}^{t} \int_{\Omega_{s}^{\varepsilon}}\left|\frac{d A}{d \mathcal{F}}\right|\left|\frac{d \mathcal{F}}{d \tau}\right| D\left(\partial_{\tau} \mathbf{u}^{\varepsilon}\right): D\left(\partial_{\tau} \mathbf{u}^{\varepsilon}\right) d x d \tau \\
& +\frac{1}{2 \varepsilon^{2}} \int_{\Omega_{s}^{\varepsilon}}\left|\frac{d A}{d \mathcal{F}}\right|\left|\frac{d \mathcal{F}}{d t}\right|\left|D\left(\mathbf{u}^{\varepsilon}(t)\right)\right|\left|D\left(\partial_{t} \mathbf{u}^{\varepsilon}(t)\right)\right| \\
& \frac{1}{2 \varepsilon^{2}} \int_{0}^{t} \int_{\Omega_{s}^{\varepsilon}}\left|\frac{d^{2} A}{d \mathcal{F}^{2}}\left(\frac{d \mathcal{F}}{d \tau}\right)^{2}+\frac{d A}{d \mathcal{F}} \frac{d^{2} \mathcal{F}}{d \tau^{2}}\right|\left|D\left(\mathbf{u}^{\varepsilon}\right)\right|\left|D\left(\partial_{\tau} \mathbf{u}^{\varepsilon}\right)\right| \\
& +\frac{C}{\varepsilon^{2}}\left\{|| D\left(\partial_{t} \mathbf{u}^{\varepsilon}(t)\right)\left\|_{L^{2}\left(\Omega_{s}^{\varepsilon}\right)^{9}}+\int_{0}^{t}\right\| D\left(\partial_{\tau} \mathbf{u}^{\varepsilon}\right) \|_{\left.L^{2}\left(\Omega_{s}^{\varepsilon}\right)^{9} d \tau\right\}}\right\} \\
& +\frac{C}{\varepsilon} \int_{0}^{t}\left\|D\left(\partial_{\tau}^{2} \mathbf{u}^{\varepsilon}\right)\right\|_{L^{2}\left(\Omega_{s}^{\varepsilon}\right)^{9} d \tau}
\end{aligned}
$$

Now, the first estimate in (4.9) together with Poincaré's inequality yield

$$
\begin{aligned}
& \left\|\partial_{t t} \mathbf{u}^{\varepsilon}\right\|_{L^{\infty}\left(0, T ; L^{2}(\Omega)\right)}+\left\|D\left(\partial_{t t} \mathbf{u}^{\varepsilon}\right)\right\|_{\left.L^{2}\left((0, T) \times \Omega_{f}^{\varepsilon}\right)\right)} \leq \frac{C}{\varepsilon}, \\
& \left\|D\left(\partial_{t} \mathbf{u}^{\varepsilon}\right)\right\|_{L^{\infty}\left(0, T ; L^{2}\left(\Omega_{s}^{\varepsilon}\right)\right)} \leq C .
\end{aligned}
$$

The estimates (4.8), (4.10), and (4.12) on the higher time derivatives are obtained by differentiating (4.23) two more times with respect to $t$, and using similar estimates as above.

Next, we will get improved estimates for $\partial_{t} u^{\varepsilon}$. These estimates will play an important role for the estimation of the transport terms in the equation for $c_{2}^{\varepsilon}$, and for the compactness arguments needed to pass to the limit $\varepsilon \rightarrow 0$.

Proposition 4.4. The following estimates hold with a constant $C$ independent of $\varepsilon$

$$
\begin{aligned}
& \left\|\partial_{t} \mathbf{u}^{\varepsilon}\right\|_{L^{2}\left((0, T) \times \Omega_{f}^{\varepsilon}\right)^{3}}+\left\|\partial_{t} \mathbf{u}^{\varepsilon}\right\|_{L^{\infty}\left(0, T ; L^{6}\left(\Omega_{s}^{\varepsilon}\right)\right)^{3}} \leq C \\
& \left\|\partial_{t t} \mathbf{u}^{\varepsilon}\right\|_{L^{2}\left((0, T) \times \Omega_{f}^{\varepsilon}\right)^{3}}+\left\|\partial_{t t} \mathbf{u}^{\varepsilon}\right\|_{L^{\infty}\left(0, T ; L^{6}\left(\Omega_{s}^{\varepsilon}\right)\right)^{3}} \leq C \\
& \left\|\partial_{t t t} \mathbf{u}^{\varepsilon}\right\|_{L^{2}\left((0, T) \times \Omega_{f}^{\varepsilon}\right)^{3}}+\left\|\partial_{t t t} \mathbf{u}^{\varepsilon}\right\|_{L^{\infty}\left(0, T ; L^{6}\left(\Omega_{s}^{\varepsilon}\right)\right)^{3}} \leq C
\end{aligned}
$$

Proof. Since $\Omega_{s}^{\varepsilon}$ is a connected Lipschitz domain, we can apply the extension result from [1] to find an extension of $\left.\partial_{t} \mathbf{u}^{\varepsilon}\right|_{\Omega_{s}^{\varepsilon}}$ to the domain $\Omega$. We denote this extension by $\partial_{t} \tilde{U}^{\varepsilon}$, and remark that $\partial_{t} \tilde{U}^{\varepsilon}$ is zero at the lateral boundary $\partial \Omega_{s}^{\varepsilon} \cap \Gamma_{3}$. Thus, using the properties of the extension, and Poincaré's and Korn's inequality on $\Omega$, we obtain for all $t \in(0, T)$

$$
\left\|\partial_{t} \tilde{U}^{\varepsilon}(t)\right\|_{\left.L^{2}(\Omega)^{3}\right)} \leq C\left\|D\left(\partial_{t} \tilde{U}^{\varepsilon}(t)\right)\right\|_{\left.L^{2}(\Omega)^{9}\right)} \leq C\left\|D\left(\partial_{t} \mathbf{u}^{\varepsilon}(t)\right)\right\|_{L^{2}\left(\Omega_{s}^{\varepsilon}\right)^{9}}
$$

Estimate (4.36) together with the second estimate in (4.9) yield

$$
\left\|\partial_{t} \tilde{U}^{\varepsilon}\right\|_{L^{\infty}\left(0, T ; H^{1}(\Omega)^{3}\right)} \leq C
$$


and due to the continuous embedding of $H^{1}(\Omega)$ into $L^{6}(\Omega)$, we obtain

$$
\left\|\partial_{t} \tilde{U}^{\varepsilon}\right\|_{L^{\infty}\left(0, T ; L^{6}(\Omega)^{3}\right)} \leq C .
$$

Since $\partial_{t} \tilde{U}^{\varepsilon}$ coincides with $\partial_{t} \mathbf{u}^{\varepsilon}$ on $\Omega_{s}^{\varepsilon}$, we conclude that

$$
\left\|\partial_{t} \mathbf{u}^{\varepsilon}\right\|_{L^{\infty}\left(0, T ; L^{6}\left(\Omega_{s}^{\varepsilon}\right)^{3}\right)} \leq C .
$$

Next, we prove the estimate for $\partial_{t} \mathbf{u}^{\varepsilon}$ on $\Omega_{f}^{\varepsilon}$. We note that $\tilde{U}^{\varepsilon} \neq \mathbf{u}^{\varepsilon}$ on $\Omega_{f}^{\varepsilon}$. However, due to the continuity of the velocities on $\Gamma^{\varepsilon}$, we have that

$$
\partial_{t} \tilde{U}^{\varepsilon}-\partial_{t} \mathbf{u}^{\varepsilon}=0, \quad \text { on } \Gamma^{\varepsilon} \times(0, T) .
$$

Furthermore, the estimates from Proposition 4.3, together with properties of the extension $\tilde{U}^{\varepsilon}$ yield

$$
\left\|D\left(\partial_{t} \tilde{U}^{\varepsilon}-\partial_{t} \mathbf{u}^{\varepsilon}\right)\right\|_{L^{2}\left((0, T) \times \Omega_{f}^{\varepsilon}\right)^{9}} \leq \frac{C}{\varepsilon} .
$$

Now, Poincaré's inequality for a periodic, porous medium of size $\varepsilon$, implies

$$
\left\|\partial_{t} \tilde{U}^{\varepsilon}-\partial_{t} \mathbf{u}^{\varepsilon}\right\|_{L^{2}\left((0, T) \times \Omega_{f}^{\varepsilon}\right)^{3}} \leq C \varepsilon\left\|D\left(\partial_{t} \tilde{U}^{\varepsilon}-\partial_{t} \mathbf{u}^{\varepsilon}\right)\right\|_{L^{2}\left((0, T) \times \Omega_{f}^{\varepsilon}\right)^{9}} \leq C .
$$

Thus, from (4.37), and (4.42), we can conclude that

$$
\left\|\partial_{t} \mathbf{u}^{\varepsilon}\right\|_{L^{2}\left((0, T) \times \Omega_{f}^{\varepsilon}\right)^{3}} \leq C .
$$

This proves the proposition.

We complete the estimates for the fluid/structure problem with the estimate for the pressure.

Proposition 4.5. We consider the following extension of the pressure $p^{\varepsilon}$ to $\Omega \times(0, T)$

$$
\tilde{p}^{\varepsilon}(x, t)= \begin{cases}p^{\varepsilon}(x, t), & (x, t) \in \Omega_{f}^{\varepsilon} \times(0, T) \\ 0, & (x, t) \in \Omega_{s}^{\varepsilon} \times(0, T)\end{cases}
$$

Then the following estimates hold

$$
\left\|\tilde{p}^{\varepsilon}\right\|_{L^{2}(\Omega \times(0, T))}+\left\|\partial_{t} \tilde{p}^{\varepsilon}\right\|_{L^{2}(\Omega \times(0, T))}+\left\|\partial_{t t} \tilde{p}^{\varepsilon}\right\|_{L^{2}(\Omega \times(0, T))} \leq C .
$$

Proof. Testing the fluid/structure interaction problem (2.9) - (2.17) by $\varphi \in$ $H^{1}(\Omega)$, we obtain for a.e. $t \in(0, T)$ :

$$
\begin{aligned}
& \int_{\Omega} \tilde{p}^{\varepsilon}(t) \operatorname{div} \varphi d x=\int_{\Omega_{f}^{\varepsilon}} p^{\varepsilon}(t) \operatorname{div} \varphi d x \\
& =\varepsilon^{2} \int_{\Omega} \partial_{t t} \mathbf{u}^{\varepsilon}(t) \varphi d x+2 \varepsilon^{2} \int_{\Omega_{f}^{\varepsilon}} D\left(\partial_{t} \mathbf{u}^{\varepsilon}(t)\right): D(\varphi) d x \\
& +\int_{\Omega_{s}^{\varepsilon}} A\left(\mathcal{F}\left(c_{1}^{\varepsilon}\right)\right) D\left(\mathbf{u}^{\varepsilon}(t)\right): D(\varphi)-\int_{\Gamma_{2}}\left(\mathcal{P}_{1}^{\varepsilon}, \mathcal{S}_{2}^{\varepsilon}, \mathcal{S}_{3}^{\varepsilon}\right) \varphi d S
\end{aligned}
$$

We note that for all $g \in L^{2}(\Omega)$, there is $\varphi \in H^{1}(\Omega)^{3}$, with $\varphi=0$ on the lateral boundary, such that $\operatorname{div} \varphi=g$, and $\|\varphi\|_{H^{1}(\Omega)} \leq C\|g\|_{L^{2}(\Omega)}$, see e.g. [17]. Hence, using 
the estimates from Proposition 4.3, and (4.45), we have for all $g \in L^{2}(\Omega \times(0, T))$ the following estimate

$$
\left|\int_{0}^{T} \int_{\Omega} \tilde{p}^{\varepsilon}(x, t) g(x, t) d x d t\right| \leq C|| \varphi\left\|_{L^{2}\left(0, T ; H^{1}(\Omega)\right)} \leq C\right\| g \|_{L^{2}(\Omega \times(0, T))} .
$$

This yields

$$
\left\|\tilde{p}^{\varepsilon}\right\|_{L^{2}(\Omega \times(0, T))} \leq C
$$

We continue by showing uniform estimates for the derivatives of the concentrations $c_{1}^{\varepsilon}$, and $c_{2}^{\varepsilon}$.

Proposition 4.6. For the concentration $c_{2}^{\varepsilon}$ the following estimates are valid with a constant $C$ independent of $\varepsilon$

$$
\begin{aligned}
& \left\|c_{2}^{\varepsilon}\right\|_{L^{\infty}\left(0, T ; L^{2}(\Omega)\right)}+\left\|\nabla c_{2}^{\varepsilon}\right\|_{L^{2}((0, T) \times \Omega)} \leq C . \\
& \left\|\left(\chi_{\Omega_{f}^{\varepsilon}}+\frac{1}{K} \chi_{\Omega_{s}^{\varepsilon}}\right) \partial_{t} c_{2}^{\varepsilon}\right\|_{L^{2}\left(0, T ;\left(W_{0}^{1,3}(\Omega)\right)^{\prime}\right)} \leq C .
\end{aligned}
$$

Proof. The estimate for $\nabla c_{2}^{\varepsilon}$ needs some special ideas, due to the presence of the transport term. Let us test equation (2.32) with $\zeta=\hat{c}_{2}^{\varepsilon}:=c_{2}^{\varepsilon}-c_{2 D}$, and obtain

$$
\begin{aligned}
& \frac{1}{2} \frac{d}{d t} \int_{\Omega}\left(\chi_{\Omega_{f}^{\varepsilon}}+\frac{1}{K} \chi_{\Omega_{s}^{\varepsilon}}\right)\left|\hat{c}_{2}^{\varepsilon}(t)\right|^{2} d x+d_{2} \int_{\Omega}\left(\chi_{\Omega_{f}^{\varepsilon}}+\frac{1}{K} \chi_{\Omega_{s}^{\varepsilon}}\right)\left|\nabla \hat{c}_{2}^{\varepsilon}(t)\right|^{2} d x \\
& -\int_{\Omega_{f}^{\varepsilon}} \partial_{t} \mathbf{u}^{\varepsilon} \hat{c}_{2}^{\varepsilon} \nabla \hat{c}_{2}^{\varepsilon} d x= \\
& -\int_{\Omega}\left(\chi_{\Omega_{f}^{\varepsilon}}+\frac{1}{K} \chi_{\Omega_{s}^{\varepsilon}}\right) \partial_{t} c_{2 D} \hat{c}_{2}^{\varepsilon} d x-d_{2} \int_{\Omega}\left(\chi_{\Omega_{f}^{\varepsilon}}+\frac{1}{K} \chi_{\Omega_{s}^{\varepsilon}}\right) \nabla c_{2 D} \nabla \hat{c}_{2}^{\varepsilon} d x \\
& +\int_{\Omega_{f}^{\varepsilon}} \partial_{t} \mathbf{u}^{\varepsilon} c_{2 D} \nabla \hat{c}_{2}^{\varepsilon} d x+\int_{\Omega}\left\{g_{2}\left(c_{2}^{\varepsilon}\right) \chi_{\Omega_{f}^{\varepsilon}}+g_{3}\left(c_{1}^{\varepsilon}, c_{2}^{\varepsilon}\right) \chi_{\Omega_{s}^{\varepsilon}}\right\} \hat{c}_{2}^{\varepsilon} d x
\end{aligned}
$$

Now, we have to estimate several terms. Let us start with the transport term on $\Omega_{f}^{\varepsilon}$. Hereby, we exploit the fact that the estimates for the velocity $\partial_{t} \mathbf{u}^{\varepsilon}$ are much better on the solid part $\Omega_{s}^{\varepsilon}$ then on the fluid part $\Omega_{f}^{\varepsilon}$. Thus, we integrate by parts, and use the continuity of the velocity on the interface $\Gamma^{\varepsilon}$, to obtain:

$$
\begin{aligned}
& -\int_{0}^{t} \int_{\Omega_{f}^{\varepsilon}} \partial_{t} \mathbf{u}^{\varepsilon} \hat{c}_{2}^{\varepsilon} \nabla \hat{c}_{2}^{\varepsilon}=-\frac{1}{2} \int_{0}^{t} \int_{\Gamma^{\varepsilon}} \partial_{t} \mathbf{u}^{\varepsilon} \cdot \nu \hat{c}_{2}^{\varepsilon} \hat{c}_{2}^{\varepsilon} d S d t \\
& =\frac{1}{2} \int_{0}^{t} \int_{\Omega_{s}^{\varepsilon}} \operatorname{div}\left(\partial_{t} \mathbf{u}^{\varepsilon} \hat{c}_{2}^{\varepsilon} \hat{c}_{2}^{\varepsilon}\right) \\
& =\frac{1}{2} \int_{0}^{t} \int_{\Omega_{s}^{\varepsilon}} \operatorname{div}\left(\partial_{t} \mathbf{u}^{\varepsilon}\right) \hat{c}_{2}^{\varepsilon} \hat{c}_{2}^{\varepsilon}+\int_{0}^{t} \int_{\Omega_{s}^{\varepsilon}} \partial_{t} \mathbf{u}^{\varepsilon} \hat{c}_{2}^{\varepsilon} \nabla \hat{c}_{2}^{\varepsilon}
\end{aligned}
$$

To estimate the right hand side of (4.51), we use the Gagliardo-Nirenberg-type inequality, see e. g. inequality (2.9) on pag. 62 in [13], Young's inequality, and the 
estimates for the velocity $\partial_{t} \mathbf{u}^{\varepsilon}$ form Propositions 4.3 and 4.4. We obtain:

$$
\begin{aligned}
& \left.\left|\int_{0}^{t} \int_{\Omega_{s}^{\varepsilon}} \operatorname{div}\left(\partial_{t} \mathbf{u}^{\varepsilon}\right) \hat{c}_{2}^{\varepsilon} \hat{c}_{2}^{\varepsilon}\right| \leq C \int_{0}^{t}\left\|D\left(\partial_{t} \mathbf{u}^{\varepsilon}\right)\right\|_{L^{2}\left(\Omega_{s}^{\varepsilon}\right)}\right)\left\|\hat{c}_{2}^{\varepsilon}\right\|_{L^{4}\left(\Omega_{s}^{\varepsilon}\right)}^{2} d \tau \\
& \leq C\left\|D\left(\partial_{t} \mathbf{u}^{\varepsilon}\right)\right\|_{L^{\infty}\left(0, T ; L^{2}\left(\Omega_{s}^{\varepsilon}\right)\right)} \int_{0}^{t}\left\|\hat{c}_{2}^{\varepsilon}\right\|_{L^{2}\left(\Omega_{s}^{\varepsilon}\right)}\left\|\nabla \hat{c}_{2}^{\varepsilon}\right\|_{L^{2}\left(\Omega_{s}^{\varepsilon}\right)} d \tau \\
& \leq \delta\left\|\nabla \hat{c}_{2}^{\varepsilon}\right\|_{L^{2}\left((0, T) \times \Omega_{s}^{\varepsilon}\right)}^{2}+C(\delta)\left\|\hat{c}_{2}^{\varepsilon}\right\|_{L^{2}\left((0, T) \times \Omega_{s}^{\varepsilon}\right)}^{2},
\end{aligned}
$$

and

$$
\begin{aligned}
& \left|\int_{0}^{t} \int_{\Omega_{s}^{\varepsilon}} \partial_{t} \mathbf{u}^{\varepsilon} \hat{c}_{2}^{\varepsilon} \nabla \hat{c}_{2}^{\varepsilon}\right| \leq C \int_{0}^{t}\left\|\partial_{t} \mathbf{u}^{\varepsilon}\right\|_{L^{6}\left(\Omega_{s}^{\varepsilon}\right)}\left\|\hat{c}_{2}^{\varepsilon}\right\|_{L^{3}\left(\Omega_{s}^{\varepsilon}\right)} \mid\left\|\nabla \hat{c}_{2}^{\varepsilon}\right\|_{L^{2}\left(\Omega_{s}^{\varepsilon}\right)} \\
& \leq C\left\|\partial_{t} \mathbf{u}^{\varepsilon}\right\|_{L^{\infty}\left(0, T ; L^{6}\left(\Omega_{s}^{\varepsilon}\right)\right)} \int_{0}^{t}\left\|\hat{c}_{2}^{\varepsilon}\right\|_{L^{3}\left(\Omega_{s}^{\varepsilon}\right)}\left\|\nabla \hat{c}_{2}^{\varepsilon}\right\|_{L^{2}\left(\Omega_{s}^{\varepsilon}\right)} d \tau \\
& \leq C \int_{0}^{t}\left\|\hat{c}_{2}^{\varepsilon}\right\|_{L^{2}\left(\Omega_{s}^{\varepsilon}\right)}^{1 / 2}\left\|\nabla \hat{c}_{2}^{\varepsilon}\right\|_{L^{2}\left(\Omega_{s}^{\varepsilon}\right)}^{3 / 2} d \tau \\
& \leq \delta\left\|\nabla \hat{c}_{2}^{\varepsilon}\right\|_{L^{2}\left((0, T) \times \Omega_{s}^{\varepsilon}\right)}^{2}+C(\delta)\left\|\hat{c}_{2}^{\varepsilon}\right\|_{L^{2}\left((0, T) \times \Omega_{s}^{\varepsilon}\right)}^{2},
\end{aligned}
$$

To estimate the terms on the right hand side in (4.50), we use Hölder's inequality, the assumptions on the data, and the estimate (4.6) for $c_{1}^{\varepsilon}$. Since these estimates are standard, we omit them.

Finally, integrating with respect to time in (4.50), and collecting the estimates (4.51) - (9.5), we obtain

$$
\int_{\Omega}\left|\hat{c}_{2}^{\varepsilon}(t)\right|^{2} d x+\int_{0}^{t} \int_{\Omega}\left|\nabla \hat{c}_{2}^{\varepsilon}(t)\right|^{2} d x \leq C+C \int_{0}^{t} \int_{\Omega}\left|\hat{c}_{2}^{\varepsilon}\right|^{2} d x d t .
$$

Now, Gronwall's lemma yields the estimate (4.48) for $c_{2}^{\varepsilon}$.

Let us now prove the estimate for the time derivative. To this end, we test equation (2.32) with $\zeta \in L^{2}\left(0, T ; W_{0}^{1,3}(\Omega)\right)$. After integration with respect to time, we obtain:

$$
\begin{aligned}
& \int_{0}^{T} \int_{\Omega}\left\{\chi_{\Omega_{f}^{\varepsilon}}+\frac{1}{K} \chi_{\Omega_{s}^{\varepsilon}}\right\} \partial_{t} c_{2}^{\varepsilon} \zeta d x d t=-\int_{0}^{T} \int_{\Omega} d_{2}\left\{\chi_{\Omega_{f}^{\varepsilon}}+\frac{1}{K} \chi_{\Omega_{s}^{\varepsilon}}\right\} \nabla c_{2}^{\varepsilon} \nabla \zeta d x d t \\
& +\int_{0}^{T} \int_{\Omega_{f}^{\varepsilon}} \partial_{t} \mathbf{u}^{\varepsilon} c_{2}^{\varepsilon} \nabla \zeta d x d t+\int_{0}^{T} \int_{\Omega}\left\{g_{2}\left(c_{2}^{\varepsilon}\right) \chi_{\Omega_{f}^{\varepsilon}}+g_{3}\left(c_{1}^{\varepsilon}, c_{2}^{\varepsilon}\right) \chi_{\Omega_{s}^{\varepsilon}}\right\} \zeta d x d t
\end{aligned}
$$

We have the following estimates

$$
\begin{aligned}
&\left|\int_{0}^{T} \int_{\Omega} d_{2}\left\{\chi_{\Omega_{f}^{\varepsilon}}+\frac{1}{K} \chi_{\Omega_{s}^{\varepsilon}}\right\} \nabla c_{2}^{\varepsilon} \nabla \zeta\right| \leq C|| \nabla c_{2}^{\varepsilon}\left\|_{L^{2}((0, T) \times \Omega)}\right\| \nabla \zeta \|_{L^{2}((0, T) \times \Omega)}, \\
& \mid\left|\int_{0}^{T} \int_{\Omega_{f}^{\varepsilon}} \partial_{t} \mathbf{u}^{\varepsilon} c_{2}^{\varepsilon} \nabla \zeta d x d t\right| \\
& \leq \int_{0}^{T}\left\|\partial_{t} \mathbf{u}^{\varepsilon}\right\|_{L^{2}\left(\Omega_{f}^{\varepsilon}\right)}|| c_{2}^{\varepsilon}\left\|_{L^{6}(\Omega)}\right\| \nabla \zeta \|_{L^{3}(\Omega)} d t \\
& \leq\left\|\partial_{t} \mathbf{u}^{\varepsilon}\right\|_{L^{\infty}\left(0, T ; L^{2}\left(\Omega_{f}^{\varepsilon}\right)\right)}\left\|c_{2}^{\varepsilon}\right\|_{L^{2}\left(0, T ; L^{6}(\Omega)\right)}|| \nabla \zeta \|_{L^{2}\left(0, T ; L^{3}(\Omega)\right)},
\end{aligned}
$$


and

$$
\begin{aligned}
& \left|\int_{0}^{T} \int_{\Omega}\left\{g_{2}\left(c_{2}^{\varepsilon}\right) \chi_{\Omega_{f}^{\varepsilon}}+g_{3}\left(c_{1}^{\varepsilon}, c_{2}^{\varepsilon}\right) \chi_{\Omega_{s}^{\varepsilon}}\right\} \zeta d x d t\right| \\
& \leq C \int_{0}^{T} \int_{\Omega}\left(1+\chi_{\Omega_{s}^{\varepsilon}}\left|c_{1}^{\varepsilon}\right|+\left|c_{2}^{\varepsilon}\right|\right) \zeta d x d t \\
& \leq C\left(1+\left\|c_{1}^{\varepsilon}\right\|_{\left.L^{2}\left((0, T) \times \Omega_{s}^{\varepsilon}\right)\right)}+\left\|c_{2}^{\varepsilon}\right\|_{L^{2}((0, T) \times \Omega)}\right)\|\zeta\|_{L^{2}((0, T) \times \Omega)}
\end{aligned}
$$

Thus, for all $\zeta \in L^{2}\left(0, T ; W_{0}^{1,3}(\Omega)\right)$ we have

$$
\left|\int_{0}^{T} \int_{\Omega}\left\{\chi_{\Omega_{f}^{\varepsilon}}+\frac{1}{K} \chi_{\Omega_{s}^{\varepsilon}}\right\} \partial_{t} c_{2}^{\varepsilon} \zeta d x d t\right| \leq C\|\zeta\|_{L^{2}\left(0, T ; W_{0}^{1,3}(\Omega)\right)},
$$

and estimate (4.49) is proved.

For every $t \in[0, T]$, let us now denote by $\tilde{c}_{1}^{\varepsilon}(t)$ the $H^{1}$-extension of $c_{1}^{\varepsilon}(t)$ to $\Omega$, as in [1]. Due to the properties of the extension operator, we have that $\tilde{c}_{1}^{\varepsilon} \in$ $L^{2}\left(0, T ; H^{1}(\Omega)\right)$, and $\partial_{t} \tilde{c}_{1}^{\varepsilon} \in L^{2}((0, T) \times \Omega)$.

Proposition 4.7. The following estimates are valid with a constant $C$ independent of $\varepsilon$

$$
\begin{aligned}
& \left\|\tilde{c}_{1}^{\varepsilon}\right\|_{L^{\infty}\left(0, T ; L^{2}(\Omega)\right)}+\left\|\nabla \tilde{c}_{1}^{\varepsilon}\right\|_{L^{2}((0, T) \times \Omega)} \leq C, \\
& \left\|\chi_{\Omega_{s}^{\varepsilon}} \partial_{t} \tilde{c}_{1}^{\varepsilon}\right\|_{L^{2}\left(0, T ; H^{-1}(\Omega)\right)} \leq C .
\end{aligned}
$$

Proof. Estimate (4.59) is immediately obtained by testing equation (2.31) by $c_{1}^{\varepsilon}$, and then using the properties of the extension operator. For the estimate on the time derivative, we observe that $\partial_{t} \tilde{c}_{1}^{\varepsilon}$ satisfies the following equation

$$
\int_{\Omega} \chi_{\Omega_{s}^{\varepsilon}} \partial_{t} \tilde{c}_{1}^{\varepsilon} \psi d x+\int_{\Omega} \chi_{\Omega_{s}^{\varepsilon}} d_{1}\left(c_{2}^{\varepsilon}\right) \nabla \tilde{c}_{1}^{\varepsilon}(t) \nabla \psi d x=\int_{\Omega} \chi_{\Omega_{s}^{\varepsilon}} g_{1}\left(\tilde{c}_{1}^{\varepsilon}, c_{2}^{\varepsilon}\right) \psi d x
$$

for all $\psi \in H_{0}^{1}(\Omega)$, and consequently we have

$$
\begin{aligned}
& \left|\int_{0}^{T} \int_{\Omega} \chi_{\Omega_{s}^{\varepsilon}} \partial_{t} \tilde{c}_{1}^{\varepsilon} \psi d x d t\right| \leq C\left\|\nabla c_{1}^{\varepsilon}\right\|_{L^{2}\left((0, T) \times \Omega_{s}^{\varepsilon}\right)}\|\nabla \psi\|_{L^{2}((0, T) \times \Omega)} \\
& +C\left(1+\left\|c_{1}^{\varepsilon}\right\|_{L^{2}\left((0, T) \times \Omega_{s}^{\varepsilon}\right.}+\left\|c_{2}^{\varepsilon}\right\|_{L^{2}\left((0, T) \times \Omega_{s}^{\varepsilon}\right)}\right)\|\psi\|_{L^{2}((0, T) \times \Omega)} \\
& \leq C\|\psi\|_{L^{2}\left(0, T ; H^{1}(\Omega)\right)}
\end{aligned}
$$

Thus, the proposition is proved.

5. Compactness of the microscopic solutions. Based on the estimates form Section 4, we prove compactness of the microscopic solutions, with respect to twoscale convergence, and with respect to weak and strong $L^{2}$-convergence. The results concerning two-scale compactness are standard, and can be found e.g. in [3] or [6]. We will not repeat the proof here. Contrary to this, the strong compactness of the concentrations is nonstandard, due to the very low regularity of the concentrations with respect to the time variable. Thus, we have to generalize the well known compactness theorem by Aubin and Lions to be able to deal with the situation given in our problem. This is done in Lemma 5.1, and Proposition 5.2 in the beginning of this section. 
LEMma 5.1. Let $a^{\varepsilon}$ be a uniformly bounded sequence in $L^{\infty}(\Omega)$, such that $a^{\varepsilon} \rightarrow a$ weakly in $L^{3}(\Omega)$, with $a \in L^{\infty}(\Omega), a(x)>0$ for a.e. $x \in \Omega$. Further, let $m>0$, and $\eta>0$ be given real numbers.

Then there exist $\gamma_{\eta}>0$, and $\varepsilon_{0}>0$, such that for all $c \in H^{1}(\Omega)$, and all $\varepsilon \leq \varepsilon_{0}$, we have

$$
\|c\|_{L^{2}(\Omega)} \leq \eta\|c\|_{H^{1}(\Omega)}+\gamma_{\eta}\left\|a^{\varepsilon} c\right\|_{H^{-m}(\Omega)} .
$$

Proof. Let us suppose that (5.1) is not true, and let $\varepsilon_{0}^{j}$, be a sequence with $\lim _{j \rightarrow \infty} \varepsilon_{0}^{j}=0$. Then, to every $\varepsilon_{0}^{j}$, there exists $\varepsilon^{j} \leq \varepsilon_{0}^{j}$, and $c_{j} \in H^{1}(\Omega), c_{j} \neq 0$ such that

$$
\left\|c_{j}\right\|_{L^{2}(\Omega)} \geq \eta\left\|c_{j}\right\|_{H^{1}(\Omega)}+j\left\|a^{\varepsilon^{j}} c_{j}\right\|_{H^{-m}(\Omega)} .
$$

Set

$$
w_{j}:=\frac{c_{j}}{\left\|c_{j}\right\|_{H^{1}(\Omega)}} .
$$

Obviously, we have $\left\|w_{j}\right\|_{H^{1}(\Omega)}=1$, and from (5.2) it follows that

$$
\left\|w_{j}\right\|_{L^{2}(\Omega)} \geq \eta+j\left\|a^{\varepsilon^{j}} w_{j}\right\|_{H^{-m}(\Omega)} .
$$

Since $\left\|w_{j}\right\|_{L^{2}(\Omega)} \leq 1$, the relation (5.3) yields

$$
a^{\varepsilon^{j}} w_{j} \rightarrow 0 \quad \text { strongly in } H^{-m}(\Omega) .
$$

On the other hand, after passing to a subsequence, we have that

$$
w_{j} \rightarrow w \quad \text { strongly in } L^{2}(\Omega) .
$$

Taking into account the weak convergence of $a^{\varepsilon^{j}} \rightarrow a$ in $L^{3}(\Omega)$, we obtain that

$$
a^{\varepsilon^{j}} w_{j} \rightarrow a w \quad \text { weakly in } L^{2}(\Omega) .
$$

Due to the compact embedding of $L^{2}(\Omega)$ into $H^{-m}(\Omega),(5.4)$, and (5.5) imply $a w=0$, and due to $a>0$, we have $w=0$. Clearly, this contradicts (5.3), and the lemma is proved. $\mathrm{C}$

Proposition 5.2. Let $a^{\varepsilon}$ be a uniformly bounded sequence in $L^{\infty}(\Omega)$, such that $a^{\varepsilon} \rightarrow a$ weakly in $L^{3}(\Omega)$, with $a \in L^{\infty}(\Omega), a(x)>0$ a.e. in $\Omega$. Furthermore, let $c^{\varepsilon}$ be a sequence in $L^{2}\left(0, T ; H^{1}(\Omega)\right)$, and assume that $a^{\varepsilon} \partial_{t} c^{\varepsilon} \in L^{2}\left(0, T ; H^{-m}(\Omega)\right)$, with $m \geq 1$.

If we have that

$$
\begin{aligned}
c^{\varepsilon} & \rightarrow c \quad \text { weakly in } L^{2}\left(0, T ; H^{1}(\Omega)\right), \\
a^{\varepsilon} \partial_{t} c^{\varepsilon} & \rightarrow a \partial_{t} c \quad \text { weakly in } L^{2}\left(0, T ; H^{-m}(\Omega)\right),
\end{aligned}
$$

then it follows that

$$
c^{\varepsilon} \rightarrow c \quad \text { strongly in } L^{2}((0, T) \times \Omega) \text {. }
$$


Proof. For the sequences $a^{\varepsilon}$, and $c^{\varepsilon}$, Lemma 5.1 implies that for all $\eta>0$ there exists $\gamma_{\eta}$, such that

$$
\left.\left\|\left(c^{\varepsilon}-c\right)(t)\right\|_{L^{2}(\Omega)} \leq \eta\left\|\left(c^{\varepsilon}-c\right)(t)\right\|_{H^{1}(\Omega)}+\gamma_{\eta} \| a^{\varepsilon}\left(c^{\varepsilon}-c\right)(t)\right) \|_{H^{-m}(\Omega)},
$$

for a. e. $t \in(0, T)$.

Let $\delta>0$ be given. Integrating (5.9) with respect to time and taking into account that $\left\|c^{\varepsilon}-c\right\|_{L^{2}\left(0, T ; H^{1}(\Omega)\right)} \leq C_{1}$, we obtain

$$
\left\|c^{\varepsilon}-c\right\|_{L^{2}((0, T) \times \Omega)} \leq \frac{\delta}{2}+\sqrt{2} \gamma_{\eta}\left\|a^{\varepsilon}\left(c^{\varepsilon}-c\right)\right\|_{L^{2}\left(0, T ; H^{-m}(\Omega)\right)},
$$

where $\eta$ was chosen such that $\sqrt{2} \eta C_{1} \leq \frac{\delta}{2}$. Hence it remains to prove that

$$
\lim _{\varepsilon \rightarrow 0}\left\|a^{\varepsilon}\left(c^{\varepsilon}-c\right)\right\|_{L^{2}\left(0, T ; H^{-m}(\Omega)\right)}=0 .
$$

We split the sequence as follows

$$
\begin{aligned}
\left\|a^{\varepsilon}\left(c^{\varepsilon}-c\right)\right\|_{L^{2}\left(0, T ; H^{-m}(\Omega)\right)} & \leq\left\|a^{\varepsilon} c^{\varepsilon}-a c\right\|_{L^{2}\left(0, T ; H^{-m}(\Omega)\right)} \\
& +\left\|\left(a^{\varepsilon}-a\right) c\right\|_{L^{2}\left(0, T ; H^{-m}(\Omega)\right)} .
\end{aligned}
$$

To show the convergence of the first term on the right hand side of (5.12), we use the information (5.7) about the time derivative. Thus, since $a^{\varepsilon} c^{\varepsilon}-a c$ is bounded in $H^{1}\left(0, T ; H^{-m}(\Omega)\right)$, we have

$$
\left\|a^{\varepsilon} c^{\varepsilon}-a c\right\|_{C\left([0, T], H^{-m}(\Omega)\right)} \leq C .
$$

Therefore, it is enough to prove that

$$
\left(a^{\varepsilon} c^{\varepsilon}-a c\right)(t) \rightarrow 0 \quad \text { in } H^{-m}(\Omega), \text { f.a.e. } t \in[0, T] .
$$

By (5.13), and (5.14) Lebesgue's dominated convergence theorem then implies

$$
\lim _{\varepsilon \rightarrow 0}\left\|a^{\varepsilon} c^{\varepsilon}-a c\right\|_{L^{2}\left(0, T ; H^{-m}(\Omega)\right)}=0 .
$$

We write $a^{\varepsilon} c^{\varepsilon}-a c$ as follows:

$$
\begin{aligned}
\left(a^{\varepsilon} c^{\varepsilon}-a c\right)(t) & =\frac{1}{s-t} \int_{t}^{s}\left(a^{\varepsilon} c^{\varepsilon}-a c\right)(\xi) d \xi \\
& \left.-\frac{1}{s-t} \int_{t}^{s}(s-\xi) \partial_{\xi}\left(a^{\varepsilon} c^{\varepsilon}-a c\right)(\xi)\right) d \xi
\end{aligned}
$$

for $s>t$. Then, using (5.7), the second term can be estimated as follows:

$$
\begin{aligned}
& \left\|\frac{1}{s-t} \int_{t}^{s}(s-\xi) \partial_{\xi}\left(a^{\varepsilon} c^{\varepsilon}-a c\right)(\xi) d \xi\right\|_{H^{-m}(\Omega)} \\
& \leq \int_{t}^{s}\left\|\partial_{\xi}\left(a^{\varepsilon} c^{\varepsilon}-a c\right)(\xi)\right\|_{H^{-m}(\Omega)} d \xi \leq \frac{\delta}{2},
\end{aligned}
$$

for $s$ sufficiently close to $t$. To estimate the first term in (5.16), we consider

$$
\frac{1}{s-t} \int_{t}^{s}\left(a^{\varepsilon} c^{\varepsilon}-a c\right)(\xi) d \xi=\frac{1}{s-t} \int_{t}^{s}\left[a^{\varepsilon}\left(c^{\varepsilon}-c\right)+\left(a^{\varepsilon}-a\right) c\right](\xi) d \xi
$$


By Lebesgue's Differentiation theorem, we have that

$$
\lim _{s \rightarrow t_{+}} \frac{1}{s-t} \int_{t}^{s}\left(c^{\varepsilon}-c\right)(\xi) d \xi=\left(c^{\varepsilon}-c\right)(t) .
$$

Hence $\frac{1}{s-t} \int_{t}^{s}\left(c^{\varepsilon}-c\right)(\xi) d \xi$ is bounded in $H^{1}(\Omega)$ and

$$
\frac{1}{s-t} \int_{t}^{s}\left(c^{\varepsilon}-c\right)(\xi) d \xi \rightarrow 0, \quad \text { weakly in } H^{1}(\Omega) .
$$

By compactness of the embedding of $H^{1}(\Omega)$ into $L^{2}(\Omega)$, we have that $\frac{1}{s-t} \int_{t}^{s}\left(c^{\varepsilon}-\right.$ $c)(\xi) d \xi$ converges strongly to zero in $L^{2}(\Omega)$, and thus the product

$$
a^{\varepsilon} \frac{1}{s-t} \int_{t}^{s}\left(c^{\varepsilon}-c\right)(\xi) d \xi \rightarrow 0, \quad \text { weakly in } L^{2}(\Omega) .
$$

Finally, due to the compact embedding of $L^{2}(\Omega)$ into $H^{-m}(\Omega)$, we obtain

$$
a^{\varepsilon} \frac{1}{s-t} \int_{t}^{s}\left(c^{\varepsilon}-c\right)(\xi) d \xi \rightarrow 0, \quad \text { strongly in } H^{-m}(\Omega) .
$$

The convergence

$$
\left(a^{\varepsilon}-a\right) \frac{1}{s-t} \int_{t}^{s} c(\xi) d \xi \rightarrow 0, \quad \text { strongly in } H^{-m}(\Omega)
$$

is straightforward, and thus (5.15) is proved.

The convergence to zero of the second term on the right hand side of (5.12) follows by an approximation argument and the fact that $a^{\varepsilon}-a$ does not depend on time. Thus, (5.11) holds, and the theorem is proved.

TheOREM 5.3. There exist limit functions $\mathbf{u}^{0} \in C^{3}\left([0, T], H^{1}(\Omega)\right)^{3}, \mathbf{u}^{1} \in C^{3}([0, T]$; $\left.L^{2}\left(\Omega ; H_{p e r}^{1}\left(Y_{s}\right)^{3} / \mathbb{R}\right)\right), \mathbf{w}^{0} \in C^{3}\left([0, T] ; L^{2}\left(\Omega ; H_{p e r}^{1}\left(Y_{f}\right)^{3}\right)\right) \cap L^{2}\left((0, T) \times \Omega ; L^{2}(Y, \operatorname{div})^{3}\right)$, and $p^{0} \in L^{2}((0, T) \times \Omega \times Y)$, such that up to a subsequence, the following convergences hold in the two scale sense:

$$
\begin{aligned}
& \mathbf{u}^{\varepsilon} \longrightarrow \mathbf{u}^{0}(t, x)+\chi_{Y_{f}} \mathbf{w}^{0}(t, x, y) \\
& \partial_{t} \mathbf{u}^{\varepsilon} \rightarrow \partial_{t} \mathbf{u}^{0}(t, x)+\chi_{Y_{f}} \partial_{t} \mathbf{w}^{0}(t, x, y) \\
& \partial_{t t} \mathbf{u}^{\varepsilon} \rightarrow \partial_{t t} \mathbf{u}^{0}(t, x)+\chi_{Y_{f}} \partial_{t t} \mathbf{w}^{0}(t, x, y) \\
& \partial_{t t t} \mathbf{u}^{\varepsilon} \rightarrow \partial_{t t t} \mathbf{u}^{0}(t, x)+\chi_{Y_{f}} \partial_{t t t} \mathbf{w}^{0}(t, x, y) \\
& \chi_{\Omega_{s}^{\varepsilon}} D\left(\mathbf{u}^{\varepsilon}\right) \rightarrow \chi_{Y_{s}}\left[D_{x}\left(\mathbf{u}^{0}(t, x)\right)+D_{y}\left(\mathbf{u}^{1}(t, x, y)\right)\right] \\
& \chi_{\Omega_{s}^{\varepsilon}} D\left(\partial_{t} \mathbf{u}^{\varepsilon}\right) \rightarrow \chi_{Y_{s}}\left[D_{x}\left(\partial_{t} \mathbf{u}^{0}(t, x)\right)+D_{y}\left(\partial_{t} \mathbf{u}^{1}(t, x, y)\right)\right]
\end{aligned}
$$

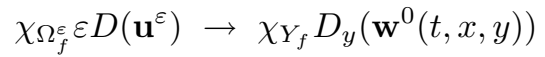

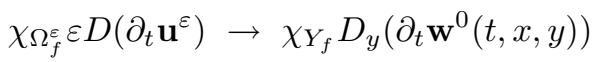

$$
\begin{aligned}
& \tilde{p^{\varepsilon}} \rightarrow p^{0}(t, x, y) \text {. }
\end{aligned}
$$

TheOREM 5.4. There exist limit functions $c_{1}^{0} \in L^{2}\left(0, T ; H^{1}(\Omega)\right), c_{2}^{0} \in L^{2}\left(0, T ; H^{1}(\Omega)\right)$, $c_{1}^{1} \in L^{2}\left((0, T) \times \Omega ; H_{\text {per }}^{1}(Y) / \mathbb{R}\right)$, and $c_{2}^{1} \in L^{2}\left((0, T) \times \Omega ; H_{\text {per }}^{1}(Y) / \mathbb{R}\right)$, such that up to 
a subsequence, the following convergence properties hold:

$$
\begin{aligned}
\tilde{c}_{1}^{\varepsilon} & \rightarrow c_{1}^{0}(t, x) \quad \text { in two-scale sense } \\
\nabla \tilde{c}_{1}^{\varepsilon} & \rightarrow \nabla_{x} c_{1}^{0}(t, x)+\nabla_{y} c_{1}^{1}(t, x, y) \quad \text { in two-scale sense } \\
\tilde{c}_{1}^{\varepsilon} & \rightarrow c_{1}^{0}(t, x) \quad \text { strongly in } L^{2}((0, T) \times \Omega) \\
\tilde{c}_{1}^{\varepsilon} & \rightarrow c_{1}^{0}(t, x) \quad \text { weakly in } L^{2}\left(0, T ; H^{1}(\Omega)\right) \\
c_{2}^{\varepsilon} & \rightarrow c_{2}^{0}(t, x) \quad \text { in two-scale sense } \\
\nabla c_{2}^{\varepsilon} & \rightarrow \nabla_{x} c_{2}^{0}(t, x)+\nabla_{y} c_{2}^{1}(t, x, y) \quad \text { in two-scale sense } \\
c_{2}^{\varepsilon} & \rightarrow c_{2}^{0}(t, x) \quad \text { strongly in } L^{2}((0, T) \times \Omega) \\
c_{2}^{\varepsilon} & \rightarrow c_{2}^{0}(t, x) \quad \text { weakly in } L^{2}\left(0, T ; H^{1}(\Omega)\right),
\end{aligned}
$$

where for every $t \in[0, T], \tilde{c}_{1}^{\varepsilon}$ is the $H^{1}$-extension of $c_{1}^{\varepsilon}$ to $\Omega$, as in [1].

Proof. To show the strong convergences (5.32), and (5.36) of the concentrations, we remark that for a subsequence,

$$
\begin{aligned}
\tilde{c}_{1}^{\varepsilon} & \rightarrow c_{1}^{0} \quad \text { weakly in } L^{2}\left(0, T ; H^{1}(\Omega)\right), \\
\chi_{\Omega_{s}^{\varepsilon}} \partial_{t} \tilde{c}_{1}^{\varepsilon} & \rightarrow\left|Y_{s}\right| \partial_{t} c_{1}^{0} \quad \text { weakly in } L^{2}\left(0, T ; H^{-1}(\Omega)\right),
\end{aligned}
$$

and

$$
\begin{aligned}
c_{2}^{\varepsilon} & \rightarrow c_{2}^{0} \quad \text { weakly in } L^{2}\left(0, T ; H^{1}(\Omega)\right), \\
\left(\chi_{\Omega_{f}^{\varepsilon}}+\frac{1}{K} \chi_{\Omega_{s}^{\varepsilon}}\right) \partial_{t} c_{2}^{\varepsilon} & \rightarrow\left(\left|Y_{f}\right|+\frac{1}{K}\left|Y_{s}\right|\right) \partial_{t} c_{2}^{0} \quad \text { weakly in } L^{2}\left(0, T ; H^{-2}(\Omega)\right),
\end{aligned}
$$

for $\varepsilon \rightarrow 0$. To verify that $\chi_{\Omega_{s}^{\varepsilon}} \partial_{t} \tilde{c}_{1}^{\varepsilon} \rightarrow\left|Y_{s}\right| \partial_{t} c_{1}^{0}$ weakly in $L^{2}\left(0, T ; H^{-1}(\Omega)\right)$, we consider $\varphi \in C_{0}^{\infty}((0, T) \times \Omega)$ and calculate

$$
<\chi_{\Omega_{s}^{\varepsilon}} \partial_{t} \tilde{c}_{1}^{\varepsilon}, \varphi>=-\int_{0}^{T} \int_{\Omega} \chi_{\Omega_{s}^{\varepsilon}} \tilde{c}_{1}^{\varepsilon} \partial_{t} \varphi=\int_{0}^{T} \int_{\Omega} \chi_{\Omega_{s}^{\varepsilon}} \int_{0}^{t} \tilde{c}_{1}^{\varepsilon} \partial_{\tau \tau} \varphi d \tau .
$$

Thus, we have that

$$
<\chi_{\Omega_{s}^{\varepsilon}} \partial_{t} \tilde{c}_{1}^{\varepsilon}, \varphi>\rightarrow \int_{0}^{T} \int_{\Omega}\left|Y_{s}\right| \int_{0}^{t} c_{1}^{0} \partial_{\tau \tau} \varphi d \tau=<\left|Y_{s}\right| \partial_{t} c_{1}^{0}, \varphi>
$$

Convergence (5.39), together with estimate (4.60) yield (5.38). The corresponding convergence for $c_{2}^{\varepsilon}$ can be proved analogously. Thus, by Proposition 5.2 the strong convergences (5.32), and (5.36) follow.

6. Passing to the limit in the microscopic equations. In this section, we pass to the limit in the weak formulation of the microscopic equations (2.9)-(2.27), for $\varepsilon \rightarrow 0$. Using the compactness results from Theorem 5.3, and Theorem 5.4, and choosing suitable test-functions, first a two-scale homogenized system is derived. In a second step, the microscopic variable can be eliminated yielding the homogenized system, with effective coefficients computed by means of cell-problems. The arguments concerning the fluid-structure interaction are similar to those used in [6], however the nonlinear dependence of the elasticity coefficients on the concentration $c_{1}^{\varepsilon}$ leads to additional difficulties. Thus, especially the strong convergence for the concentrations, proved in Theorem 5.4, will be of big advantage. We recall that the outer unit normal to $\partial \Omega$ is denoted by $\nu$. We also denote by $\nu$ the outer unit normal to $\partial Y_{s}$ (i.e. the unit normal exterior to the solid structure). 
Proposition 6.1. The limit functions $\mathbf{u}^{0}, \mathbf{u}^{1}, p^{0}$, and $c_{1}^{0}$, defined in Theorem 5.3, and Theorem 5.4, satisfy for all $(t, x) \in(0, T) \times \Omega$ the following equations

$$
\begin{aligned}
& -\operatorname{Div}_{y}\left\{\mathcal{A}\left(\mathcal{F}\left(c_{1}^{0}\right)(t, x)\right)\left(D_{x}\left(\mathbf{u}^{0}\right)+D_{y}\left(\mathbf{u}^{1}\right)\right)\right\}=0 \text { in } Y_{s} \\
& \mathcal{A}\left(\mathcal{F}\left(c_{1}^{0}\right)(t, x)\right)\left(D_{x}\left(\mathbf{u}^{0}\right)+D_{y}\left(\mathbf{u}^{1}\right)\right) \cdot \nu+p^{0}(t, x, y) \cdot \nu=0 \text { on } \partial Y_{s} \backslash \partial Y \\
& \nabla_{y} p^{0}=0 \text { in } Y_{f}, \quad \text { and } \quad p^{0}=0 \text { in } Y_{s}, \\
& \mathbf{u}^{1}, p^{0} \text { are } Y-\text { periodic }
\end{aligned}
$$

Furthermore, there exists $p_{f} \in L^{2}((0, T) \times \Omega)$ such that

$$
p^{0}(t, x, y)=p_{f}(t, x) \chi_{Y_{f}}(y) \text {. }
$$

Proposition 6.2. We have

$$
\begin{aligned}
& -\operatorname{Div}_{x}\left\{\int_{Y_{s}} \mathcal{A}\left(\mathcal{F}\left(c_{1}^{0}\right)\right)\left(D_{x}\left(\mathbf{u}^{0}\right)+D_{y}\left(\mathbf{u}^{1}\right)\right) d y\right\}+\left|Y_{f}\right| \nabla_{x} p_{f}(t, x)=0 \\
& \text { in }(0, T) \times \Omega \\
& \left(\int_{Y_{s}} \mathcal{A}\left(\mathcal{F}\left(c_{1}^{0}\right)\right)\left(D_{x}\left(\mathbf{u}^{0}\right)+D_{y}\left(\mathbf{u}^{1}\right)\right) d y-\left|Y_{f}\right| p_{f}(t, x) I\right) \cdot \mathbf{e}_{1} \\
& =\left\{\begin{array}{ccc}
0, & \text { on } & (0, T) \times \Gamma_{1} \\
\left(\mathcal{P}^{0},\left|F_{s}\right| \mathcal{C}_{2}^{s},\left|F_{s}\right| \mathcal{C}_{3}^{s}\right), & \text { on } & (0, T) \times \Gamma_{2}
\end{array}\right. \\
& \mathbf{u}^{0}(t, x)=0 \quad \text { on }(0, T) \times \Gamma_{3} \text {. }
\end{aligned}
$$

Corollary 6.3. For all $(t, x) \in(0, T) \times \Omega$, let $w^{i j}(t, x, y)$ and $\gamma^{p}(t, x, y)$ be the solutions to the following problems

$$
\begin{aligned}
& -\operatorname{Div}_{y}\left\{\mathcal{A}\left(\mathcal{F}\left(c_{1}^{0}\right)\right)\left(\frac{\mathbf{e}_{i} \mathbf{e}_{j}+\mathbf{e}_{j} \mathbf{e}_{i}}{2}+D_{y}\left(w^{i j}\right)\right)\right\}=0 \quad \text { in } Y_{s} \\
& \mathcal{A}\left(\mathcal{F}\left(c_{1}^{0}\right)\right)\left(\frac{\mathbf{e}_{i} \mathbf{e}_{j}+\mathbf{e}_{j} \mathbf{e}_{i}}{2}+D_{y}\left(w^{i j}\right)\right) \cdot \nu=0 \quad \text { on } \partial Y_{s} \backslash \partial Y \\
& w^{i j} \text { is } Y \text {-periodic, }
\end{aligned}
$$

and

$$
\begin{aligned}
& -\operatorname{Div}_{y}\left(\mathcal{A}\left(\mathcal{F}\left(c_{1}^{0}\right)\right) D_{y}\left(\gamma^{p}\right)\right)=0 \quad \text { in } Y_{s} \\
& \mathcal{A}\left(\mathcal{F}\left(c_{1}^{0}\right)\right) D_{y}\left(\gamma^{p}\right) \cdot \nu+\nu=0 \quad \text { on } \partial Y_{s} \backslash \partial Y \\
& \gamma^{p} \text { is } Y-\text { periodic. }
\end{aligned}
$$

Then, we have

$$
\mathbf{u}^{1}(t, x, y)=\sum_{i, j=1}^{3}\left(D_{x}\left(\mathbf{u}^{0}(t, x)\right)\right)_{i j} w^{i j}(t, x, y)+p_{f}(t, x) \gamma^{p}(t, x, y) .
$$

Furthermore, the effective elastic moduli coefficient $\mathcal{A}^{H}$, given by

$$
\mathcal{A}_{k l m n}^{H}(t, x)=\sum_{i, j=1}^{3} \int_{Y_{s}} \mathcal{A}_{k l i j}\left(\mathcal{F}\left(c_{1}^{0}\right)\right)\left(\delta_{i m} \delta_{j n}+\left(D_{y}\left(w^{m n}(t, x, y)\right)\right)_{i j}\right) d y,
$$


is a symmetric and positive definite 4 th order tensor.

Proposition 6.4. We have the following two-scale variant of Darcy's law. There exists $\pi^{0} \in L^{2}\left((0, T) \times \Omega \times Y_{f}\right)$ such that the limit function $\mathbf{w}^{0}$ from Theorem 5.3, and $p_{f}$ given by (6.5) satisfy

$$
\begin{aligned}
& -\Delta_{y}\left(\partial_{t} \mathbf{w}^{0}\right)+\nabla_{y} \pi^{0}=-\nabla_{x} p_{f} \quad \text { in }(0, T) \times \Omega \times Y_{f} \\
& \operatorname{div}_{y}\left(\partial_{t} \mathbf{w}^{0}\right)=0 \quad \text { in }(0, T) \times \Omega \times Y_{f} \\
& \partial_{t} \mathbf{w}^{0}=0 \quad \text { on }(0, T) \times \Omega \times\left(\partial Y_{f} \backslash \partial Y\right) \\
& \mathbf{w}^{0}, \pi^{0} \text { are } Y-\text { periodic }
\end{aligned}
$$

Furthermore, $\nabla p_{f} \in L^{2}((0, T) \times \Omega)$, and $p_{f}$ satisfies the following boundary conditions on the boundaries $\Gamma_{1}$, and $\Gamma_{2}$.

$$
\begin{aligned}
& p_{f}=0 \quad \text { on }(0, T) \times \Gamma_{1} \\
& p_{f}=\mathcal{P}^{0} \quad \text { on }(0, T) \times \Gamma_{2} .
\end{aligned}
$$

Proof. We start from the weak formulation (2.28), and by standard arguments, see e.g. the seminal paper [3], we obtain equations (6.17) - (6.20). We remark that (6.17) implies $\nabla p_{f} \in L^{2}((0, T) \times \Omega)$. To obtain the boundary conditions for $p_{f}$, we insert in (2.28) the test-function $\varphi(t, x)=\varepsilon^{2} \zeta\left(x, \frac{x}{\varepsilon}\right) h(t)$ with $\zeta \in C^{\infty}\left(\Omega ; C_{p e r}^{\infty}(Y)\right)^{3}, \zeta(x, y)=0$ on $\left(\Omega \times Y_{s}\right) \cup\left(\Gamma_{3} \times Y_{f}\right)$, and $\operatorname{div}_{y} \zeta=0$ in $\Omega \times Y_{f}$. Let $h \in C_{0}^{\infty}(0, T)$. We have

$$
\begin{aligned}
& \varepsilon^{2} \int_{0}^{T} \int_{\Omega} \partial_{t t} \mathbf{u}^{\varepsilon}(t) \zeta\left(x, \frac{x}{\varepsilon}\right) h(t) d x d t \\
+ & 2 \int_{0}^{T} \int_{\Omega} \varepsilon \chi_{\Omega_{f}^{\varepsilon}} D\left(\partial_{t} \mathbf{u}^{\varepsilon}(t)\right):\left(\varepsilon D_{x}(\zeta)\left(x, \frac{x}{\varepsilon}\right)+D_{y}(\zeta)\left(x, \frac{x}{\varepsilon}\right)\right) h(t) d x d t+ \\
+ & \int_{0}^{T} \int_{\Omega} \chi_{\Omega_{f}^{\varepsilon}} \tilde{p}^{\varepsilon} \operatorname{div}_{x} \zeta\left(x, \frac{x}{\varepsilon}\right) h(t) d x d t \\
= & \int_{0}^{T} \int_{\Gamma_{2}}\left(\mathcal{P}_{1}^{\varepsilon}, \mathcal{S}_{2}^{\varepsilon}, \mathcal{S}_{3}^{\varepsilon}\right) \zeta\left(x, \frac{x}{\varepsilon}\right) h(t) d S
\end{aligned}
$$

We pass to the limit $\varepsilon \rightarrow 0$ using the two-scale compactness from Theorem 5.3, and the properties of the boundary data. It yields

$$
\begin{aligned}
& 2 \int_{0}^{T} \int_{\Omega} \int_{Y_{f}} D_{y}\left(\partial_{t} \mathbf{w}^{0}\right)(t, x, y): D_{y}(\zeta)(x, y) h(t) d y d x d t \\
& +\int_{0}^{T} \int_{\Omega} \int_{Y_{f}} p_{f}(t, x) \operatorname{div}_{x} \zeta(x, y) h(t) d y d x d t \\
& =\int_{0}^{T} \int_{\Gamma_{2}} \int_{F_{f}}\left(\mathcal{P}^{0}(t, x), 0,0\right) \zeta(x, y) h(t) d y d S d t .
\end{aligned}
$$

Let now $\zeta(x, y)=\psi(x) \eta(y)$, with $\psi \in C^{\infty}(\Omega), \psi=0$ on $\Gamma_{3}$, and $\eta \in C_{p e r}^{\infty}(Y)^{3}, \eta=0$ on $Y_{s}, \operatorname{div}_{y} \eta=0$ in $Y_{f}$. Integrating by parts in (6.24), and using equations (6.17) (6.20), we obtain

$$
\begin{aligned}
& \int_{0}^{T} \int_{\Gamma_{1} \cup \Gamma_{2}} p_{f}(t, x) \psi(x) h(t) \int_{Y_{f}} \eta(y) d y \cdot \nu d S d t \\
& =\int_{0}^{T} \int_{\Gamma_{2}}\left(\mathcal{P}^{0}(t, x), 0,0\right) \psi(x) h(t) \int_{F_{f}} \eta(y) d y d S d t .
\end{aligned}
$$


From here (6.21) follows immediately. To get (6.22), we use the $Y$-periodicity of $\eta$, and the fact that $\operatorname{div}_{y} \eta=0$ in $Y_{f}$. This implies that for all $l \in[0,1]$, we have

$$
\int_{y_{1}=l} \eta_{1}(y) d y=\int_{F_{f}} \eta_{1}(y) d y .
$$

Recall that $F_{s}$ was defined in (2.1). Thus taking $\psi=0$ on $\Gamma_{1}$, we obtain form (6.25)

$$
\begin{aligned}
& \int_{0}^{T} \int_{\Gamma_{2}} p_{f}(t, x) \psi(x) h(t) \int_{Y_{f}} \eta_{1}(y) d y d S d t \\
& =\int_{0}^{T} \int_{\Gamma_{2}} \mathcal{P}^{0}(t, x) \psi(x) h(t) \int_{F_{f}} \eta_{1}(y) d y d S d t
\end{aligned}
$$

and using (6.26), we get the boundary condition (6.22).

口

Corollary 6.5. Let $\left\{\chi^{j}, \pi^{j}\right\}$ be the solution to

$$
\begin{aligned}
& -\Delta_{y} \chi^{j}+\nabla_{y} \pi^{j}=\mathbf{e}_{j} \quad \text { in } Y_{f} \\
& \operatorname{div}_{y} \chi^{j}=0 \quad \text { in } Y_{f} \\
& \mathbf{w}^{0}=0 \quad \text { on } \partial Y_{f} \backslash \partial Y \\
& \chi^{j}, \pi^{j} \text { are } Y-\text { periodic }
\end{aligned}
$$

Then, we have

$$
\begin{aligned}
& \partial_{t} \mathbf{w}^{0}(t, x, y)=-\sum_{j=1}^{3} \chi^{j}(y) \frac{\partial p_{f}(t, x)}{\partial x_{j}}, \\
& \pi^{0}(t, x, y)=-\sum_{j=1}^{3} \pi^{j}(y) \frac{\partial p_{f}(t, x)}{\partial x_{j}}
\end{aligned}
$$

and the permeability tensor $K$ given by

$$
K_{i j}=\int_{Y_{f}} \chi_{i}^{j}(y) d y
$$

is a positive definite symmetric tensor.

Proposition 6.6. The limit functions $\mathbf{u}^{0}, \mathbf{w}^{0}$, and $\mathbf{u}^{1}$ from Theorem 5.3 are linked by the continuity equation

$$
\begin{aligned}
& \operatorname{div}_{x}\left(\left|Y_{f}\right| \partial_{t} \mathbf{u}^{0}(t, x)+\int_{Y_{f}} \partial_{t} \mathbf{w}^{0}(t, x, y) d y\right)=\int_{Y_{s}} \operatorname{div}_{y} \partial_{t} \mathbf{u}^{1}(t, x, y) d y \\
& \int_{Y_{f}} \partial_{t} \mathbf{w}^{0} d y \cdot \nu=0 \quad \text { on }(0, T) \times \Gamma_{3}
\end{aligned}
$$


Proposition 6.7. The limit functions $c_{1}^{0}$, and $c_{1}^{1}$ from Theorem 5.4 satisfy the following equations

$$
\begin{aligned}
& -\operatorname{div}_{y}\left\{d_{1}\left(c_{2}^{0}\right)\left(\nabla_{x} c_{1}^{0}(t, x)+\nabla_{y} c_{1}^{1}(t, x, y)\right)\right\}=0 \quad \text { in }(0, T) \times \Omega \times Y_{s} \\
& d_{1}\left(c_{2}^{0}\right)\left(\nabla_{x} c_{1}^{0}+\nabla_{y} c_{1}^{1}\right) \cdot \nu=0 \quad \text { on }(0, T) \times \Omega \times\left(\partial Y_{s} \backslash \partial Y\right) \\
& c_{1}^{1} \text { is } Y-\text { periodic } \\
& \left|Y_{s}\right| \partial_{t} c_{1}^{0}-\operatorname{div}_{x}\left(\int_{Y_{s}} d_{1}\left(c_{2}^{0}\right)\left(\nabla_{x} c_{1}^{0}(t, x)+\nabla_{y} c_{1}^{1}(t, x, y)\right) d y\right) \\
& =\left|Y_{s}\right| g\left(c_{1}^{0}, c_{2}^{0}\right) \quad \text { on }(0, T) \times \Omega \\
& \int_{Y_{s}} d_{1}\left(c_{2}^{0}\right)\left(\nabla_{x} c_{1}^{0}(t, x)+\nabla_{y} c_{1}^{1}(t, x, y)\right) d y \cdot \nu=0 \quad \text { on }(0, T) \times \partial \Omega \\
& c_{1}^{0}(0, x)=c_{10} \quad \text { in } \Omega,
\end{aligned}
$$

where the limit function $c_{2}^{0}$ is given in Proposition 5.4.

Corollary 6.8. Let $\left\{\omega^{j}\right\}$ be the solution to

$$
\begin{aligned}
& -\Delta_{y} \omega^{j}=0 \quad \text { in } Y_{s} \\
& \left(\nabla_{y} \omega^{j}+\mathbf{e}_{j}\right) \cdot \nu=0 \quad \text { on } \partial Y_{s} \backslash \partial Y \\
& \omega^{j} \text { is } Y-\text { periodic }
\end{aligned}
$$

Then, we have

$$
c_{1}^{1}(t, x, y)=\sum_{j=1}^{3} \omega^{j}(y) \frac{\partial c_{1}^{0}(t, x)}{\partial x_{j}} .
$$

Furthermore, the matrix $\beta$ given by

$$
\beta_{i j}=\int_{Y_{s}}\left(\frac{\partial \omega^{j}}{\partial y_{i}}(y)+\delta_{i j}\right) d y
$$

is symmetric and positive definite.

Proposition 6.9. Let $k(y):=\chi_{Y_{f}}(y)+\frac{1}{K} \chi_{Y_{s}}(y)$. The limit functions $c_{2}^{0}$, and $c_{2}^{1}$ from Theorem 5.4 satisfy the following equations

$$
\begin{aligned}
& -\operatorname{div}_{y}\left\{k(y)\left(\nabla_{x} c_{2}^{0}(t, x)+\nabla_{y} c_{2}^{1}(t, x, y)\right)-\chi_{Y_{f}}(y) \partial_{t} \mathbf{u}^{0} c_{2}^{0}\right\}=0 \\
& \text { in }(0, T) \times \Omega \times Y \\
& c_{1}^{2} \text { is } Y \text { - periodic } \\
& \left(\int_{Y} k(y) d y\right) \partial_{t} c_{2}^{0}-d_{2} d i v_{x}\left(\int_{Y} k(y)\left(\nabla_{x} c_{2}^{0}(t, x)+\nabla_{y} c_{2}^{1}(t, x, y)\right) d y\right) \\
& +\operatorname{div}_{x}\left(\left(\left|Y_{f}\right| \partial_{t} \mathbf{u}^{0}+\int_{Y_{f}} \partial_{t} \mathbf{w}^{0}\right) c_{2}^{0}\right) \\
& =\left|Y_{s}\right| g_{2}\left(c_{1}^{0}, c_{2}^{0}\right)+\left|Y_{f}\right| g_{3}\left(c_{2}^{0}\right) \quad \text { in }(0, T) \times \Omega \\
& \left(d_{2} \int_{Y} k(y)\left(\nabla_{x} c_{2}^{0}+\nabla_{y} c_{2}^{1}\right) d y-\left(\left|Y_{f}\right| \partial_{t} \mathbf{u}^{0}+\int_{Y_{f}} \partial_{t} \mathbf{w}^{0}\right) c_{2}^{0}\right) \cdot \nu=0 \\
& \text { on }(0, T) \times \Gamma_{3} \\
& c_{2}^{0}=c_{2 D} \quad \text { on }(0, T) \times\left(\Gamma_{1} \cup \Gamma_{2}\right) \\
& c_{2}^{0}(0, x)=c_{20} \quad \text { in } \Omega \text {. }
\end{aligned}
$$


Corollary 6.10. Let $\left\{\eta^{j}, \lambda^{j}\right\}, j=1,2,3$, be the solutions to the following problems

$$
\begin{aligned}
& -\operatorname{div}_{y}\left\{k(y)\left(\mathbf{e}_{j}+\nabla_{y} \eta^{j}\right)\right\}=0 \quad \text { in } Y \\
& \eta^{j} \text { is } Y \text {-periodic }
\end{aligned}
$$

and

$$
\begin{aligned}
& -\operatorname{div}_{y}\left\{k(y) \nabla_{y} \lambda^{j}-\chi_{Y_{f}}(y) \mathbf{e}_{j}\right\}=0 \quad \text { in } Y \\
& \lambda^{j} \text { is } Y \text {-periodic }
\end{aligned}
$$

Then, we have

$$
c_{2}^{1}(t, x, y)=\sum_{j=1}^{3} \eta^{j}(y) \frac{\partial c_{2}^{0}}{\partial x_{j}}(t, x)+\sum_{j=1}^{3} \lambda^{j}(y) \partial_{t} \mathbf{u}^{0}(t, x) \mathbf{e}_{j} c_{2}^{0}(t, x) .
$$

Furthermore, the matrix $d_{2} \int_{Y} k(y) \Upsilon(y) d y$, where

$$
\Upsilon_{i j}(y)=\frac{\partial \eta^{j}}{\partial y_{i}}(y)+\delta_{i j}
$$

is symmetric and positive definite.

6.1. Variational formulation of the homogenized system. The variational formulation for the effective fluid-structure problem is the following:

Find $\mathbf{u}^{0} \in C^{1}\left([0, T], H^{1}(\Omega)\right)^{3}, \partial_{t t} \mathbf{u}^{0} \in L^{2}\left(Q_{T}\right), \partial_{t} \mathbf{u}^{1} \in L^{2}\left(Q_{T}, H_{p e r}^{1}\left(Y_{s}\right) / \mathbb{R}\right)^{3}, \partial_{t} \mathbf{w}^{0} \in$ $L^{2}\left(Q_{T}, H_{\text {per }}^{1}\left(Y_{f}\right)\right)^{3} \cap L^{2}\left(Q_{T}, L^{2}\left(Y_{f}, \operatorname{div}\right)\right)^{3}, p_{f} \in L^{2}\left(Q_{T}\right)$, such that

$$
\begin{aligned}
& \int_{\Omega} \int_{Y_{s}} \mathcal{A}\left(\mathcal{F}\left(c_{1}^{0}\right)\right)\left(D_{x}\left(\mathbf{u}^{0}\right)+D_{y}\left(\mathbf{u}^{1}\right)\right) D_{x}(\varphi) d y d x \\
& -\int_{\Omega} \int_{Y_{f}} p_{f}(t, x) \operatorname{div}_{x} \varphi d y d x=\int_{\Gamma_{2}}\left(\mathcal{P}^{0},\left|F_{s}\right| \mathcal{C}_{2}^{s},\left|F_{s}\right| \mathcal{C}_{3}^{s}\right) \varphi d S, \\
& \varphi \in H^{1}(\Omega)^{3}, \varphi=0 \text { on } \Gamma_{3}, \\
& \int_{\Omega} \int_{Y_{s}} \mathcal{A}\left(\mathcal{F}\left(c_{1}^{0}\right)\right)\left(D_{x}\left(\mathbf{u}^{0}\right)+D_{y}\left(\mathbf{u}^{1}\right)\right) D_{y}(\psi)-\int_{\Omega} \int_{Y_{f}} p_{f} \operatorname{div}_{y} \psi d y=0, \\
& \psi \in L^{2}\left(\Omega, H_{p e r}^{1}(Y)\right)^{3}, \\
& 2 \int_{\Omega} \int_{Y_{f}} D_{y}\left(\partial_{t} \mathbf{w}^{0}\right): D_{y}(\zeta) d x d y-\int_{\Omega} \int_{Y_{f}} p_{f} \operatorname{div}_{x} \zeta d y d x \\
& =\int_{\Gamma_{2}} \int_{F_{f}}\left(\mathcal{P}^{0}, 0,0\right) \zeta d y d S, \\
& \zeta \in L^{2}\left(\Omega, H_{p e r}^{1}\left(Y_{f}\right)\right)^{3}, \operatorname{div}_{y} \zeta=0, \text { in } Y_{f} \times \Omega, \zeta=0, \text { on } \partial Y_{f} \backslash \partial Y,
\end{aligned}
$$




$$
\begin{aligned}
& \operatorname{div}_{x}\left(\left|Y_{f}\right| \partial_{t} \mathbf{u}^{0}(t, x)+\int_{Y_{f}} \partial_{t} \mathbf{w}^{0}(t, x, y) d y\right)=\int_{Y_{s}} \operatorname{div}_{y} \partial_{t} \mathbf{u}^{1}(t, x, y) d y, \\
& \text { in }(0, T) \times \Omega, \\
& \mathbf{u}^{0}(t, x)=0, \quad \text { on }(0, T) \times \Gamma_{3}, \\
& p_{f}=0 \quad \text { on }(0, T) \times \Gamma_{1} \\
& p_{f}=\mathcal{P}^{0} \quad \text { on }(0, T) \times \Gamma_{2}, \\
& \int_{Y_{f}} \partial_{t} \mathbf{w}^{0} d y \cdot \nu=0, \quad \text { on }(0, T) \times \Gamma_{3},
\end{aligned}
$$

where the tensor $\mathcal{A}$ is given by

$$
\mathcal{A}\left(\mathcal{F}\left(c_{1}^{0}\right)\right)(x, t)=\mathcal{A}\left(\int_{0}^{t} \mathcal{K}(t-\tau) F\left(c_{1}^{0}(\tau, x)\right) d \tau\right) .
$$

These equations are valid a.e. on $(0, T)$. At $t=0$ all unknowns are equal to zero.

The homogenized concentrations $c_{1}^{0}$, and $c_{2}^{0}$, satisfy the variational formulation: Find $c_{1}^{0}, c_{2}^{0} \in L^{2}\left(0, T ; H^{1}(\Omega)\right)$, with $\partial_{t} c_{1}^{0}, \partial_{t} c_{2}^{0} \in L^{2}((0, T) \times \Omega)$, such that

$$
\begin{aligned}
& \left|Y_{s}\right| \int_{\Omega} \partial_{t} c_{1}^{0} \varphi+\int_{\Omega} d_{1} \beta \nabla c_{1}^{0}(t, x) \nabla \varphi=\left|Y_{s}\right| \int_{\Omega} g_{1}\left(c_{1}^{0}, c_{2}^{0}\right) \varphi \\
& \varphi \in H^{1}(\Omega), \\
& \left(\int_{Y} k(y) d y\right) \int_{\Omega} \partial_{t} c_{2}^{0} \psi+\int_{\Omega} d_{2}\left(\int_{Y} k(y) \Upsilon(y) d y\right) \nabla_{x} c_{2}^{0} \nabla_{x} \psi d x \\
& -\int_{\Omega}\left[\left(\left|Y_{f}\right|-\sum_{j=1}^{3} \int_{Y_{f}} d_{2} k(y) \nabla_{y} \lambda^{j}(y) e^{j} d y\right) \partial_{t} \mathbf{u}^{0}+\int_{Y_{f}} \partial_{t} \mathbf{w}^{0} d y\right] \times \\
& \times c_{2}^{0} \nabla_{x} \psi d x=\int_{\Omega}\left(\left|Y_{s}\right| g_{2}\left(c_{1}^{0}, c_{2}^{0}\right)+\left|Y_{f}\right| g_{3}\left(c_{2}^{0}\right)\right) \psi d x, \\
& \psi \in H^{1}(\Omega), \psi=0 \text { on } \Gamma_{1} \cup \Gamma_{2}, \\
& c_{2}^{0}=c_{2 D} \quad \text { on }(0, T) \times\left(\Gamma_{1} \cup \Gamma_{2}\right) \\
& c_{1}^{0}(0, x)=c_{10} \quad \text { in } \Omega \\
& c_{2}^{0}(0, x)=c_{20} \quad \text { in } \Omega .
\end{aligned}
$$

7. Convergence of energies. Let us now prove the convergence of the energies stated in Theorem 3.1.

Proof. We start from the variational formulation of the fluid-structure interaction:

$$
\begin{aligned}
& \varepsilon^{2} \int_{0}^{t} \int_{\Omega} \partial_{t t} \mathbf{u}^{\varepsilon} \varphi d x d \tau+2 \int_{0}^{t} \int_{\Omega_{f}^{\varepsilon}} \varepsilon^{2} D\left(\partial_{t} \mathbf{u}^{\varepsilon}\right): D(\varphi) d x d \tau+ \\
& \int_{0}^{t} \int_{\Omega_{s}^{\varepsilon}} A\left(\mathcal{F}\left(c_{1}^{\varepsilon}\right)\right) D\left(\mathbf{u}^{\varepsilon}\right): D(\varphi) d x d \tau-\int_{0}^{t} \int_{\Omega_{f}^{\varepsilon}} p^{\varepsilon} \operatorname{div} \varphi d x d \tau \\
& =\int_{0}^{t} \int_{\Gamma_{2}}\left(\mathcal{P}_{1}^{\varepsilon}, \mathcal{S}_{2}^{\varepsilon}, \mathcal{S}_{3}^{\varepsilon}\right) \varphi d S d \tau
\end{aligned}
$$


for all $\varphi \in L^{2}\left(0, T ; H^{1}(\Omega)\right), \varphi=0$ on $\Gamma_{3}$. Let us first insert $\varphi=\mathbf{u}^{\varepsilon}$ as test function in (7.1), and obtain

$$
\begin{aligned}
& \varepsilon^{2} \int_{0}^{t} \int_{\Omega} \partial_{t t} \mathbf{u}^{\varepsilon} \mathbf{u}^{\varepsilon} d x d \tau+\int_{\Omega_{f}^{\varepsilon}}\left|\varepsilon D\left(\mathbf{u}^{\varepsilon}\right)(t)\right|^{2} d x \\
& +\int_{0}^{t} \int_{\Omega_{s}^{\varepsilon}} A\left(\mathcal{F}\left(c_{1}^{\varepsilon}\right)\right) D\left(\mathbf{u}^{\varepsilon}\right): D\left(\mathbf{u}^{\varepsilon}\right) d x d \tau=\int_{0}^{t} \int_{\Gamma_{2}}\left(\mathcal{P}_{1}^{\varepsilon}, \mathcal{S}_{2}^{\varepsilon}, \mathcal{S}_{3}^{\varepsilon}\right) \mathbf{u}^{\varepsilon} d S d \tau .
\end{aligned}
$$

Our aim is to pass to the limit in the above equation for $\varepsilon \rightarrow 0$. To this end, we use the localization (dilation, unfolding) operator $\mathcal{T}^{\varepsilon}$, and its properties, see e.g. in [4], and [5]. Taking into account the lower-semicontinuity of the norm with respect to the weak convergence, we have for all $t \in(0, T)$

$$
\begin{aligned}
& \liminf _{\varepsilon \rightarrow 0} \int_{\Omega_{f}^{\varepsilon}}\left|\varepsilon^{2} D\left(\mathbf{u}^{\varepsilon}\right)\right|^{2}(t) d x=\liminf _{\varepsilon \rightarrow 0} \int_{\Omega} \int_{Y_{f}} \mid \varepsilon^{2} \mathcal{T}^{\varepsilon}\left(\left.D\left(\mathbf{u}^{\varepsilon}\right)\right|^{2}(t) d y d x\right. \\
& \geq \int_{\Omega} \int_{Y_{f}}\left|D_{y}\left(\mathbf{w}^{\mathbf{0}}\right)\right|^{2}(t) d y d x .
\end{aligned}
$$

Due to the strong convergence of $c_{1}^{\varepsilon}$ in $L^{2}((0, T) \times \Omega)$, the convergence in two-scales of $\chi_{\Omega_{s}^{\varepsilon}} D\left(\mathbf{u}^{\varepsilon}\right)$, see Theorem 5.3, and Theorem 5.4, as well as the properties of $A(\mathcal{F})$, we have the following convergence in two-scale sense

$$
A\left(\mathcal{F}\left(c_{1}^{\varepsilon}\right)\right)^{1 / 2} D\left(\mathbf{u}^{\varepsilon}\right) \rightarrow A\left(\mathcal{F}\left(c_{1}^{0}\right)\right)^{1 / 2}\left(D_{x}\left(\mathbf{u}^{\mathbf{0}}\right)+D_{y}\left(\mathbf{u}^{\mathbf{1}}\right)\right) .
$$

This implies for all $t \in(0, T)$

$$
\begin{aligned}
& \liminf _{\varepsilon \rightarrow 0} \int_{0}^{t} \int_{\Omega_{s}^{\varepsilon}} A\left(\mathcal{F}\left(c_{1}^{\varepsilon}\right)(t, x)\right)\left|D\left(\mathbf{u}^{\varepsilon}\right)(t, x)\right|^{2} d x d \tau \\
& =\liminf _{\varepsilon \rightarrow 0} \int_{0}^{t} \int_{\Omega} \int_{Y_{s}} \mathcal{T}^{\varepsilon}\left(A\left(\mathcal{F}\left(c_{1}^{\varepsilon}\right)\right)\right)(t, x, y)\left|\mathcal{T}^{\varepsilon}\left(D\left(\mathbf{u}^{\varepsilon}\right)\right)(t, x, y)\right|^{2} d y d x d \tau \\
& \geq \int_{0}^{t} \int_{\Omega} \int_{Y_{s}} A\left(\mathcal{F}\left(c_{1}^{0}\right)\right)\left|\left(D_{x}\left(\mathbf{u}^{\mathbf{0}}\right)+D_{y}\left(\mathbf{u}^{\mathbf{1}}\right)\right)\right|^{2} d y d x d \tau .
\end{aligned}
$$

Passing in (7.2) to the limit for $\varepsilon \rightarrow 0$, we obtain

$$
\begin{aligned}
& \int_{\Omega} \int_{Y_{f}}\left|D_{y}\left(\mathbf{w}^{\mathbf{0}}\right)\right|^{2}(t) d y d x \\
+ & \int_{0}^{t} \int_{\Omega} \int_{Y_{s}} A\left(\mathcal{F}\left(c_{1}^{0}\right)\right)\left|\left(D_{x}\left(\mathbf{u}^{\mathbf{0}}\right)+D_{y}\left(\mathbf{u}^{\mathbf{1}}\right)\right)\right|^{2} d y d x d \tau \\
\leq & \lim _{\varepsilon \rightarrow 0} \int_{0}^{t} \int_{\Gamma_{2}}\left(\mathcal{P}_{1}^{\varepsilon}, \mathcal{S}_{2}^{\varepsilon}, \mathcal{S}_{3}^{\varepsilon}\right) \mathbf{u}^{\varepsilon} d S d \tau \\
= & \int_{0}^{t} \int_{\Gamma_{2}} \int_{F_{1}} \mathcal{P}^{0}\left(\mathbf{u}_{1}^{0}+\chi_{F_{f}} \mathbf{w}_{1}^{0}\right)(t, x, y) d y d x d \tau \\
+ & \int_{0}^{t} \int_{\Gamma_{2}}\left|F_{s}\right|\left(\mathcal{C}_{2}^{s} \mathbf{u}_{2}^{0}+\mathcal{C}_{3}^{s} \mathbf{u}_{3}^{0}\right)(t, x) d x d \tau .
\end{aligned}
$$


Now, we derive an expression for the right hand side in (7.6) by using the homogenized equations. First, we test equation (6.59) with $\varphi=\mathbf{u}^{0}$ and obtain

$$
\begin{aligned}
& \int_{0}^{t} \int_{\Omega} \int_{Y_{s}} \mathcal{A}\left(\mathcal{F}\left(c_{1}^{0}\right)\right)\left(D_{x}\left(\mathbf{u}^{0}\right)+D_{y}\left(\mathbf{u}^{1}\right)\right) D_{x}\left(\mathbf{u}^{0}\right) d y d x d \tau \\
- & \int_{0}^{t} \int_{\Omega} \int_{Y_{f}} p_{f}(t, x) \operatorname{div}_{x} \mathbf{u}^{0} d y d x d \tau \\
= & \int_{0}^{t} \int_{\Gamma_{2}} \mathcal{P}^{0} \mathbf{u}_{1}^{0} d x d \tau+\int_{0}^{t} \int_{\Gamma_{2}}\left|F_{s}\right|\left(\mathcal{C}_{2}^{s} \mathbf{u}_{2}^{0}+\mathcal{C}_{3}^{s} \mathbf{u}_{3}^{0}\right)(t, x) d x d \tau .
\end{aligned}
$$

Next, we test (6.60) with $\psi=\mathbf{u}^{1}$, and get

$$
\begin{aligned}
& \int_{0}^{t} \int_{\Omega} \int_{Y_{s}} \mathcal{A}\left(\mathcal{F}\left(c_{1}^{0}\right)\right)\left(D_{x}\left(\mathbf{u}^{0}\right)+D_{y}\left(\mathbf{u}^{1}\right)\right) D_{y}\left(\mathbf{u}^{1}\right) d y d x d \tau \\
& -\int_{0}^{t} \int_{\Omega} \int_{Y_{f}} p_{f} \operatorname{div}_{y} \mathbf{u}^{1} d y d x d \tau=0 .
\end{aligned}
$$

Finally, we test (6.61) with $\zeta=\mathbf{w}^{0}$, and obtain

$$
\begin{aligned}
& \int_{\Omega} \int_{Y_{f}}\left|D_{y}\left(\mathbf{w}^{0}(t)\right)\right|^{2} d x d y-\int_{0}^{t} \int_{\Omega} \int_{Y_{f}} p_{f} \operatorname{div}_{x} \mathbf{w}^{0} d y d x d \tau \\
& =\int_{0}^{t} \int_{\Gamma_{2}} \int_{F_{f}} \mathcal{P}^{0}(\tau, x) \mathbf{w}_{1}^{0}(\tau, x, y) d y d S d \tau
\end{aligned}
$$

Adding equations (7.7), (7.8), and (7.9), and using (6.62) gives

$$
\begin{aligned}
& \int_{0}^{t} \int_{\Omega} \int_{Y_{s}} \mathcal{A}\left(\mathcal{F}\left(c_{1}^{0}\right)\right)\left(D_{x}\left(\mathbf{u}^{0}\right)+D_{y}\left(\mathbf{u}^{1}\right)\right):\left(D_{x}\left(\mathbf{u}^{0}\right)+D_{y}\left(\mathbf{u}^{1}\right) d y d x d \tau\right. \\
+ & \int_{\Omega} \int_{Y_{f}}\left|D_{y}\left(\mathbf{w}^{0}(t)\right)\right|^{2} d x d y \\
= & \int_{0}^{t} \int_{\Gamma_{2}} \int_{F_{1}} \mathcal{P}^{0}\left(\mathbf{u}_{1}^{0}+\chi_{F_{f}} \mathbf{w}_{1}^{0}\right)(\tau, x, y) d y d x d \tau \\
+ & \int_{0}^{t} \int_{\Gamma_{2}}\left|F_{s}\right|\left(\mathcal{C}_{2}^{s} \mathbf{u}_{2}^{0}+\mathcal{C}_{3}^{s} \mathbf{u}_{3}^{0}\right)(\tau, x) d x d \tau .
\end{aligned}
$$

Comparing (7.6), and (7.10), and using lower-semicontinuity of the norms, gives

$$
\begin{aligned}
& \liminf _{\varepsilon \rightarrow 0} \int_{\Omega_{f}^{\varepsilon}}\left|\varepsilon^{2} D\left(\mathbf{u}^{\varepsilon}\right)\right|^{2}(t) d x=\int_{\Omega} \int_{Y_{f}}\left|D_{y}\left(\mathbf{w}^{\mathbf{0}}\right)\right|^{2}(t) d y d x \\
& =\limsup _{\varepsilon \rightarrow 0} \int_{\Omega_{f}^{\varepsilon}}\left|\varepsilon^{2} D\left(\mathbf{u}^{\varepsilon}\right)\right|^{2}(t) d x,
\end{aligned}
$$

and

$$
\begin{aligned}
& \liminf _{\varepsilon \rightarrow 0} \int_{0}^{t} \int_{\Omega_{s}^{\varepsilon}} A\left(\mathcal{F}\left(c_{1}^{\varepsilon}\right)(\tau, x)\right)\left|D\left(\mathbf{u}^{\varepsilon}\right)(\tau, x)\right|^{2} d x d \tau \\
& =\int_{0}^{t} \int_{\Omega} \int_{Y_{s}} A\left(\mathcal{F}\left(c_{1}^{0}\right)\right)\left|\left(D_{x}\left(\mathbf{u}^{\mathbf{0}}\right)+D_{y}\left(\mathbf{u}^{\mathbf{1}}\right)\right)\right|^{2} d y d x d \tau \\
& =\limsup _{\varepsilon \rightarrow 0} \int_{0}^{t} \int_{\Omega_{s}^{\varepsilon}} A\left(\mathcal{F}\left(c_{1}^{\varepsilon}\right)(\tau, x)\right)\left|D\left(\mathbf{u}^{\varepsilon}\right)(\tau, x)\right|^{2} d x d \tau,
\end{aligned}
$$


proving (3.19), and (3.20).

To show the last two statements of the theorem, we again start from the variational formulation of the fluid-structure system (7.1), and insert $\varphi=\partial_{t} \mathbf{u}^{\varepsilon}$ as test function. We obtain

$$
\begin{aligned}
& \varepsilon^{2} \int_{0}^{t} \int_{\Omega} \partial_{\tau \tau} \partial_{\tau} \mathbf{u}^{\varepsilon} \mathbf{u}^{\varepsilon} d x d \tau+\int_{0}^{t} \int_{\Omega_{f}^{\varepsilon}} \varepsilon^{2}\left|D\left(\partial_{\mathbf{t}} \mathbf{u}^{\varepsilon}\right)(\tau)\right|^{2} d x d \tau \\
& +\frac{1}{2} \int_{\Omega_{s}^{\varepsilon}} A\left(\mathcal{F}\left(c_{1}^{\varepsilon}(t)\right)\right) D\left(\mathbf{u}^{\varepsilon}(t)\right): D\left(\mathbf{u}^{\varepsilon}(t)\right) d x \\
& -\frac{1}{2} \int_{0}^{t} \int_{\Omega_{s}^{\varepsilon}} \frac{d A}{d \mathcal{F}}\left(\mathcal{F}\left(c_{1}^{\varepsilon}\right)\right) \frac{d \mathcal{F}}{d \tau}\left(c_{1}^{\varepsilon}\right) D\left(\mathbf{u}^{\varepsilon}\right): D\left(\mathbf{u}^{\varepsilon}\right) d x d \tau \\
& =\int_{0}^{t} \int_{\Gamma_{2}}\left(\mathcal{P}_{1}^{\varepsilon}, \mathcal{S}_{2}^{\varepsilon}, \mathcal{S}_{3}^{\varepsilon}\right) \partial_{t} \mathbf{u}^{\varepsilon} d S d \tau
\end{aligned}
$$

Let us set

$$
\mathcal{C}^{\varepsilon}(t, x)=\frac{d A}{d \mathcal{F}}\left(\mathcal{F}\left(c_{1}^{\varepsilon}(t, x)\right)\right) \frac{d \mathcal{F}}{d \tau}\left(c_{1}^{\varepsilon}(t, x)\right)
$$

Then, we have

$$
\mathcal{C}^{\varepsilon} \rightarrow \mathcal{C}=\frac{d A}{d \mathcal{F}}\left(\mathcal{F}\left(c_{1}^{0}\right)\right) \frac{d \mathcal{F}}{d \tau}\left(c_{1}^{0}\right)
$$

strongly in $L^{2}((0, T) \times \Omega)$, and a.e. on $(0, T) \times \Omega$. Now, we restate (3.19) as

$$
\begin{aligned}
& \lim _{\varepsilon \rightarrow 0} \int_{0}^{T} \int_{\Omega_{s}^{\varepsilon}} A\left(\mathcal{F}\left(c_{1}^{\varepsilon}\right)\right) D\left(\mathbf{u}^{\varepsilon}\right): D\left(\mathbf{u}^{\varepsilon}\right) d x d t \\
= & \lim _{\varepsilon \rightarrow 0} \int_{0}^{T} \int_{\Omega_{s}^{\varepsilon}} \mathcal{T}^{\varepsilon}\left(A\left(\mathcal{F}\left(c_{1}^{\varepsilon}\right)\right)\right) \mathcal{T}^{\varepsilon}\left(D\left(\mathbf{u}^{\varepsilon}\right)\right): \mathcal{T}^{\varepsilon}\left(D\left(\mathbf{u}^{\varepsilon}\right)\right) d x d t \\
= & \int_{0}^{T} \int_{\Omega} \int_{Y_{s}} A\left(\mathcal{F}\left(c_{1}^{0}\right)\right)\left(D_{x}\left(\mathbf{u}^{\mathbf{0}}\right)+D_{y}\left(\mathbf{u}^{\mathbf{1}}\right)\right):\left(D_{x}\left(\mathbf{u}^{\mathbf{0}}\right)+D_{y}\left(\mathbf{u}^{\mathbf{1}}\right)\right) d y d x d t
\end{aligned}
$$

Using (7.15), we show that

$$
\lim _{\varepsilon \rightarrow 0} \int_{0}^{T} \int_{\Omega} \int_{Y_{s}}\left|\mathcal{T}^{\varepsilon}\left(D\left(\mathbf{u}^{\varepsilon}\right)\right)-D_{x}\left(\mathbf{u}^{\mathbf{0}}\right)-D_{y}\left(\mathbf{u}^{\mathbf{1}}\right)\right|^{2} d y d x d t=0 .
$$


Indeed, we have

$$
\begin{aligned}
& \int_{0}^{T} \int_{\Omega} \int_{Y_{s}}\left|\mathcal{T}^{\varepsilon}\left(D\left(\mathbf{u}^{\varepsilon}\right)\right)-D_{x}\left(\mathbf{u}^{\mathbf{0}}\right)-D_{y}\left(\mathbf{u}^{\mathbf{1}}\right)\right|^{2} d y d x d t \\
\leq & \lambda_{0} \int_{0}^{T} \int_{\Omega} \int_{Y_{s}} \mathcal{T}^{\varepsilon}\left(A\left(\mathcal{F}\left(c_{1}^{\varepsilon}\right)\right)\right)\left(\mathcal{T}^{\varepsilon}\left(D\left(\mathbf{u}^{\varepsilon}\right)\right)-D_{x}\left(\mathbf{u}^{\mathbf{0}}\right)-D_{y}\left(\mathbf{u}^{\mathbf{1}}\right)\right): \\
: & \left(\mathcal{T}^{\varepsilon}\left(D\left(\mathbf{u}^{\varepsilon}\right)\right)-D_{x}\left(\mathbf{u}^{\mathbf{0}}\right)-D_{y}\left(\mathbf{u}^{\mathbf{1}}\right)\right) d y d x d t \\
= & \lambda_{0} \int_{0}^{T} \int_{\Omega} \int_{Y_{s}} \mathcal{T}^{\varepsilon}\left(A\left(\mathcal{F}\left(c_{1}^{\varepsilon}\right)\right)\right) \mathcal{T}^{\varepsilon}\left(D\left(\mathbf{u}^{\varepsilon}\right)\right): \mathcal{T}^{\varepsilon}\left(D\left(\mathbf{u}^{\varepsilon}\right)\right) d y d x d t \\
- & \lambda_{0} \int_{0}^{T} \int_{\Omega} \int_{Y_{s}} A\left(\mathcal{F}\left(c_{1}^{0}\right)\right)\left(D_{x}\left(\mathbf{u}^{\mathbf{0}}\right)+D_{y}\left(\mathbf{u}^{\mathbf{1}}\right)\right):\left(D_{x}\left(\mathbf{u}^{\mathbf{0}}\right)+D_{y}\left(\mathbf{u}^{\mathbf{1}}\right)\right) d y d x d t \\
+ & \lambda_{0} \int_{0}^{T} \int_{\Omega} \int_{Y_{s}}\left(\mathcal{T}^{\varepsilon}\left(A\left(\mathcal{F}\left(c_{1}^{\varepsilon}\right)\right)\right)-A\left(\mathcal{F}\left(c_{1}^{0}\right)\right)\right)\left(D_{x}\left(\mathbf{u}^{\mathbf{0}}\right)+D_{y}\left(\mathbf{u}^{\mathbf{1}}\right)\right): \\
: & \left(D_{x}\left(\mathbf{u}^{\mathbf{0}}\right)+D_{y}\left(\mathbf{u}^{\mathbf{1}}\right)\right) d y d x d t \\
- & 2 \lambda_{0} \int_{0}^{T} \int_{\Omega} \int_{Y_{s}} \mathcal{T}^{\varepsilon}\left(A\left(\mathcal{F}\left(c_{1}^{\varepsilon}\right)\right)\right)\left(\mathcal{T}^{\varepsilon}\left(D\left(\mathbf{u}^{\varepsilon}\right)\right)-D_{x}\left(\mathbf{u}^{\mathbf{0}}\right)-D_{y}\left(\mathbf{u}^{\mathbf{1}}\right)\right): \\
& :\left(D_{x}\left(\mathbf{u}^{\mathbf{0}}\right)-D_{y}\left(\mathbf{u}^{\mathbf{1}}\right)\right) d y d x d t .
\end{aligned}
$$

The right hand side in (7.17) tends to zero for $\varepsilon \rightarrow 0$, since the sum of the first two terms converges to zero by (7.15), the third term converges to zero due to the strong convergence of $c_{1}^{\varepsilon}$ to $c_{1}^{0}$ in $L^{2}((0, T) \times \Omega)$, and the properties of the localization operator $\mathcal{T}^{\varepsilon}$, and finally, the forth term tends to zero due to the weak convergence of $\mathcal{T}^{\varepsilon}\left(D\left(\mathbf{u}^{\varepsilon}\right)\right)$ to its two-scale limit. Thus, (7.16) is proved.

Next, we have that

$$
\begin{aligned}
& \int_{0}^{t} \int_{\Omega_{s}^{\varepsilon}} \mathcal{C}^{\varepsilon} D\left(\mathbf{u}^{\varepsilon}\right): D\left(\mathbf{u}^{\varepsilon}\right) d x d \tau \\
& =\int_{0}^{t} \int_{\Omega} \int_{Y_{s}} \mathcal{T}^{\varepsilon}\left(\mathcal{C}^{\varepsilon}\right) \mathcal{T}^{\varepsilon}\left(D\left(\mathbf{u}^{\varepsilon}\right)\right): \mathcal{T}^{\varepsilon}\left(D\left(\mathbf{u}^{\varepsilon}\right)\right) d y d x d \tau \\
& =\int_{0}^{t} \int_{\Omega} \int_{Y_{s}} \mathcal{T}^{\varepsilon}\left(\mathcal{C}^{\varepsilon}\right)\left(\mathcal{T}^{\varepsilon}\left(D\left(\mathbf{u}^{\varepsilon}\right)\right)-D_{x}\left(\mathbf{u}^{\mathbf{0}}\right)-D_{y}\left(\mathbf{u}^{\mathbf{1}}\right)\right): \\
& :\left(\mathcal{T}^{\varepsilon}\left(D\left(\mathbf{u}^{\varepsilon}\right)\right)-D_{x}\left(\mathbf{u}^{\mathbf{0}}\right)-D_{y}\left(\mathbf{u}^{\mathbf{1}}\right)\right) d y d x d \tau \\
& +2 \int_{0}^{t} \int_{\Omega} \int_{Y_{s}} \mathcal{T}^{\varepsilon}\left(\mathcal{C}^{\varepsilon}\right) \mathcal{T}^{\varepsilon}\left(D\left(\mathbf{u}^{\varepsilon}\right)\right):\left(D_{x}\left(\mathbf{u}^{\mathbf{0}}\right)+D_{y}\left(\mathbf{u}^{\mathbf{1}}\right)\right) d y d x d \tau \\
& -\int_{0}^{t} \int_{\Omega} \int_{Y_{s}} \mathcal{T}^{\varepsilon}\left(\mathcal{C}^{\varepsilon}\right)\left(D_{x}\left(\mathbf{u}^{\mathbf{0}}\right)+D_{y}\left(\mathbf{u}^{\mathbf{1}}\right)\right):\left(D_{x}\left(\mathbf{u}^{\mathbf{0}}\right)+D_{y}\left(\mathbf{u}^{\mathbf{1}}\right)\right) d y d x d \tau
\end{aligned}
$$

Passing to the limit in (7.18), and using (7.14), (7.16), as well as generalized Lebesgue's theorem, we obtain

$$
\begin{aligned}
& \lim _{\varepsilon \rightarrow 0} \int_{0}^{t} \int_{\Omega_{s}^{\varepsilon}} \mathcal{C}^{\varepsilon} D\left(\mathbf{u}^{\varepsilon}\right): D\left(\mathbf{u}^{\varepsilon}\right) d x d \tau \\
& =\int_{0}^{t} \int_{\Omega} \int_{Y_{s}} \mathcal{C}^{0}\left(D_{x}\left(\mathbf{u}^{\mathbf{0}}\right)+D_{y}\left(\mathbf{u}^{\mathbf{1}}\right)\right):\left(D_{x}\left(\mathbf{u}^{\mathbf{0}}\right)+D_{y}\left(\mathbf{u}^{\mathbf{1}}\right)\right) d y d x d \tau
\end{aligned}
$$


Now, passing to the limit $\varepsilon \rightarrow 0$ in (7.13), we obtain

$$
\begin{aligned}
& \liminf _{\varepsilon \rightarrow 0} \int_{0}^{t} \int_{\Omega_{f}^{\varepsilon}} \varepsilon^{2}\left|D\left(\partial_{\mathbf{t}} \mathbf{u}^{\varepsilon}\right)(\tau)\right|^{2} d x d \tau \\
& +\liminf _{\varepsilon \rightarrow 0} \frac{1}{2} \int_{\Omega_{s}^{\varepsilon}} A\left(\mathcal{F}\left(c_{1}^{\varepsilon}(t)\right)\right) D\left(\mathbf{u}^{\varepsilon}(t)\right): D\left(\mathbf{u}^{\varepsilon}(t)\right) d x \\
& -\frac{1}{2} \int_{0}^{t} \int_{\Omega} \int_{Y_{s}} \mathcal{C}^{0}\left(D_{x}\left(\mathbf{u}^{\mathbf{0}}\right)+D_{y}\left(\mathbf{u}^{\mathbf{1}}\right)\right):\left(D_{x}\left(\mathbf{u}^{\mathbf{0}}\right)+D_{y}\left(\mathbf{u}^{\mathbf{1}}\right)\right) d y d x d \tau \\
& =\int_{0}^{t} \int_{\Gamma_{2}} \int_{F_{1}} \mathcal{P}^{0}\left(\partial_{t} \mathbf{u}_{1}^{0}+\chi_{F_{f}} \partial_{t} \mathbf{w}_{1}^{0}\right)(\tau, x, y) d y d x d \tau \\
& +\int_{0}^{t} \int_{\Gamma_{2}}\left|F_{s}\right|\left(\mathcal{C}_{2}^{s} \partial_{t} \mathbf{u}_{2}^{0}+\mathcal{C}_{3}^{s} \partial_{t} \mathbf{u}_{3}^{0}\right)(\tau, x) d x d \tau
\end{aligned}
$$

As in the first part of the proof, we now test the homogenized equations (6.59), (6.60), and (6.61) with $\varphi=\partial_{t} \mathbf{u}^{0}, \psi=\partial_{t} \mathbf{u}^{1}$, and $\zeta=\partial_{t} \mathbf{w}^{0}$ respectively. Adding the obtained equations gives

$$
\begin{aligned}
& 2 \int_{0}^{t} \int_{\Omega} \int_{Y_{f}}\left|D_{y}\left(\partial_{t} \mathbf{w}^{0}\right)(\tau)\right|^{2} d x d \tau \\
+ & \frac{1}{2} \int_{\Omega} \int_{Y_{f}} A\left(\mathcal{F}\left(c_{1}^{0}(t)\right)\right)\left(D_{x}\left(\mathbf{u}^{0}\right)+D_{y}\left(\mathbf{u}^{1}\right)\right):\left(D_{x}\left(\mathbf{u}^{0}\right)+D_{y}\left(\mathbf{u}^{1}\right)\right)(t) d y d x \\
- & \frac{1}{2} \int_{0}^{t} \int_{\Omega} \int_{Y_{s}} \mathcal{C}^{0}\left(D_{x}\left(\mathbf{u}^{0}\right)+D_{y}\left(\mathbf{u}^{1}\right)\right):\left(D_{x}\left(\mathbf{u}^{0}\right)+D_{y}\left(\mathbf{u}^{1}\right)\right) d y d x d \tau \\
= & \int_{0}^{t} \int_{\Gamma_{2}} \int_{F_{1}} \mathcal{P}^{0}\left(\partial_{t} \mathbf{u}_{1}^{0}+\chi_{F_{f}} \partial_{t} \mathbf{w}_{1}^{0}\right)(\tau, x, y) d y d x d \tau \\
+ & \int_{0}^{t} \int_{\Gamma_{2}}\left|F_{s}\right|\left(\mathcal{C}_{2}^{s} \partial_{t} \mathbf{u}_{2}^{0}+\mathcal{C}_{3}^{s} \partial_{t} \mathbf{u}_{3}^{0}\right)(\tau, x) d x d \tau
\end{aligned}
$$

Inserting (7.21) into (7.20) and using again the lower-semicontinuity of the norms, as well as the properties of the elasticity coefficients, we get

$$
\begin{aligned}
& 2 \int_{0}^{t} \int_{\Omega} \int_{Y_{f}}\left|D_{y}\left(\partial_{t} \mathbf{w}^{0}\right)(\tau)\right|^{2} d x d \tau \\
+ & \frac{1}{2} \int_{\Omega} \int_{Y_{f}} A\left(\mathcal{F}\left(c_{1}^{0}(t)\right)\right)\left(D_{x}\left(\mathbf{u}^{0}\right)+D_{y}\left(\mathbf{u}^{1}\right)\right):\left(D_{x}\left(\mathbf{u}^{0}\right)+D_{y}\left(\mathbf{u}^{1}\right)\right)(t) d y d x \\
\leq & \liminf _{\varepsilon \rightarrow 0} \int_{0}^{t} \int_{\Omega_{f}^{\varepsilon}} \varepsilon^{2}\left|D\left(\partial_{\mathbf{t}} \mathbf{u}^{\varepsilon}\right)(\tau)\right|^{2} d x d \tau \\
+ & \liminf _{\varepsilon \rightarrow 0} \frac{1}{2} \int_{\Omega_{s}^{\varepsilon}} A\left(\mathcal{F}\left(c_{1}^{\varepsilon}(t)\right)\right) D\left(\mathbf{u}^{\varepsilon}(t)\right): D\left(\mathbf{u}^{\varepsilon}(t)\right) d x \\
= & \int_{0}^{t} 2 \int_{\Omega} \int_{Y_{f}}\left|D_{y}\left(\partial_{t} \mathbf{w}^{0}\right)(\tau)\right|^{2} d x d \tau \\
+ & \frac{1}{2} \int_{\Omega} \int_{Y_{f}} A\left(\mathcal{F}\left(c_{1}^{0}(t)\right)\right)\left(D_{x}\left(\mathbf{u}^{0}\right)+D_{y}\left(\mathbf{u}^{1}\right)\right):\left(D_{x}\left(\mathbf{u}^{0}\right)+D_{y}\left(\mathbf{u}^{1}\right)\right)(t) d y d x
\end{aligned}
$$

From (7.22), the convergences of the energies (3.21), and (3.22) follow, and the theorem is proved. 
8. Improved regularity for the homogenized problem. In the following, we suppose $d_{1}$ independent of $c_{2}^{0}$, i.e. $d_{1}$ is a strictly positive constant. Furthermore, let $c_{10} \in C_{0}^{\infty}(\Omega)$. Let us denote by $Q_{T}=[0, T] \times \Omega$.

THEOREM 8.1. The solutions of the homogenized system have the following additional regularity properties:

$$
\begin{aligned}
c_{1}^{0} & \in W_{10 / 3}^{2,1}\left(Q_{T}\right), \\
c_{2}^{0} & \in W_{10 / 3}^{2,1}\left(Q_{T}\right) \\
p_{f} & \in H^{2}\left(0, T ; H^{1}(\Omega) \cap H^{1}\left(0, T ; H^{3}(\Omega)\right),\right. \\
\mathbf{u}^{0} & \in H^{2}\left(0, T ; H^{2}(\Omega)\right), \\
\mathbf{u}^{1} & \in H^{2}\left(0, T ; C^{1}\left(\bar{Y}_{s} ; H^{1}(\Omega)\right)\right) .
\end{aligned}
$$

Moreover, the effective transport velocity

$$
v^{H}(t, x)=\left(\left|Y_{f}\right|-\sum_{j=1}^{3} \int_{Y_{f}} d_{2} k(y) \nabla_{y} \lambda^{j}(y) e^{j} d y\right) \partial_{t} \mathbf{u}^{0}(t, x)+\int_{Y_{f}} \partial_{t} \mathbf{w}^{0}(t, x, y) d y
$$

satisfies

$$
v^{H} \in L^{\infty}\left(Q_{T}\right)
$$

Proof. For constant diffusion coefficient $d_{1}$, we have that $c_{1}^{0}$ satisfies the following problem with constant coefficients

$$
\begin{aligned}
& \left|Y_{s}\right| \partial_{t} c_{1}^{0}-\operatorname{div}_{x}\left(d_{1} \beta \nabla_{x} c_{1}^{0}\right)=\left|Y_{s}\right| g\left(c_{1}^{0}, c_{2}^{0}\right) \quad \text { on }(0, T) \times \Omega \\
& d_{1} \beta \nabla_{x} c_{1}^{0} \cdot \nu=0 \quad \text { on }(0, T) \times \partial \Omega \\
& c_{1}^{0}(0, x)=c_{10} \quad \text { in } \Omega,
\end{aligned}
$$

where the matrix $\beta$ is given by (6.47). The estimates (4.59), and (4.48), imply that $c_{1}^{0}, c_{2}^{0}$ are bounded in $L^{2}\left(0, T ; H^{1}(\Omega)\right) \cap L^{\infty}\left(0, T ; L^{2}(\Omega)\right)$. Then, by interpolation, see inequality (3.2), page 74 in [13], we obtain that

$$
g_{1}\left(c_{1}^{0}, c_{2}^{0}\right) \in L^{10 / 3}\left(Q_{T}\right), g_{2}\left(c_{1}^{0}, c_{2}^{0}\right) \in L^{10 / 3}\left(Q_{T}\right), g_{3}\left(c_{2}^{0}\right) \in L^{10 / 3}\left(Q_{T}\right) .
$$

Finally, using the parabolic regularity theory, see Theorem 9.1, page 341 from [13], we obtain $c_{1}^{0} \in W_{10 / 3}^{2,1}\left(Q_{T}\right)$. This proves (8.1). To obtain improved regularity for $c_{2}^{0}$, we first have to get improved regularity for displacements and pressure.

From the representation formula (6.32), we have

$$
\int_{Y_{f}} \partial_{t} \mathbf{w}^{0}(t, x, y) d y=-\sum_{j=1}^{3} \int_{Y_{f}} \chi^{j}(y) d y \frac{\partial p_{f}(t, x)}{\partial x_{j}} .
$$

Thus, since $\mathbf{w}^{0} \in C^{3}\left(0, T ; L^{2}\left(\Omega \times Y_{f}\right)\right)$, and the permeability tensor $K$ from (6.34) is symmetric and positive definite, we have that

$$
p_{f} \in H^{2}\left(0, T ; H^{1}(\Omega)\right) .
$$


Due to the regularity of the concentration $c_{1}^{0}$, i.e.

$$
c_{1}^{0} \in W_{10 / 3}^{2,1}\left(Q_{T}\right), \text { with } 10 / 3>3=n,
$$

we have that $c_{1}^{0} \in L^{10 / 3}\left(0, T ; C^{1}(\bar{\Omega})\right)$. Due to the definition (2.7), it follows that the components of the tensor $\mathcal{A}\left(\mathcal{F}\left(c_{1}^{0}\right)\right)$ belong to $C^{3}\left([0, T], C^{1}(\bar{\Omega})\right)$, and for the solutions $w^{i j}$ and $\gamma^{p}$ to the problems (6.9) - (6.11), respectively (6.12) - (6.14), we have

$$
w^{i j} \in C^{1}\left(\bar{Y}_{s} ; C^{3}\left([0, T], C^{1}(\bar{\Omega})\right)^{3}, \quad \gamma^{p} \in C^{1}\left(\bar{Y}_{s} ; C^{3}\left([0, T], C^{1}(\bar{\Omega})\right),\right.\right.
$$

see e.g. Theorem 9.1, page 341 in [13].

To obtain additional regularity for $\mathbf{u}^{0}$, let us write the homogenized problem in the equivalent form:

$$
\begin{aligned}
& -\operatorname{Div}_{x}\left\{\mathcal{A}^{H}(t, x) D_{x}\left(\mathbf{u}^{0}\right)\right\}=\operatorname{Div}_{x}\left\{\mathcal{B}^{H}(t, x) p_{f}(t, x)\right\} \\
& -\left|Y_{f}\right| \nabla_{x} p_{f}(t, x) \quad \text { in }(0, T) \times \Omega \\
& \mathcal{A}^{H}(t, x) D_{x}\left(\mathbf{u}^{0}\right) \cdot \nu=-p_{f}(t, x)\left(\mathcal{B}^{H}-\left|Y_{f}\right| I\right) \cdot \nu \\
& +\left\{\begin{array}{ccc}
0, & \text { on } & (0, T) \times \Gamma_{1} \\
\left(\int_{F_{1}} \mathcal{P}^{0} d y,\left|F_{s}\right| \mathcal{C}_{2}^{s},\left|F_{s}\right| \mathcal{C}_{3}^{s}\right), & \text { on } & (0, T) \times \Gamma_{2}
\end{array}\right. \\
& \mathbf{u}^{0}(t, x)=0 \quad \text { on }(0, T) \times \Gamma_{3},
\end{aligned}
$$

where the tensor $\mathcal{A}^{H}$ is given by (6.16), and

$$
\mathcal{B}^{H}(t, x)=\int_{Y_{s}} \mathcal{A}\left(\mathcal{F}\left(c_{1}^{0}(t, x)\right) D_{y}\left(\gamma^{p}(t, x, y)\right) d y .\right.
$$

Extending the right hand side in (8.16) to $\Omega$, and including it in the right hand side in (8.15), we obtain homogeneous boundary conditions on $\Gamma_{1} \cup \Gamma_{2}$. Then, extending the solution by uneven reflection with respect to $\Gamma_{3}$, and using elliptic regularity, see e.g. [2], we obtain

$$
\mathbf{u}^{0} \in H^{2}\left(0, T ; H^{2}(\Omega)\right),
$$

which proves (8.4). Moreover, using the representation formula (6.15), together with (8.4), (8.3), and (8.14), we obtain (8.5).

To obtain the second part of (8.3), we remark that the representation formula (6.32) together with the continuity equation (6.35) yield

$$
\begin{aligned}
& -\operatorname{div}_{x}\left(K \nabla p_{f}\right)=-\operatorname{div}_{x}\left(\left|Y_{f}\right| \partial_{t} \mathbf{u}^{0}\right)+\int_{Y_{s}} \operatorname{div}_{y} \partial_{t} \mathbf{u}^{1} d y \\
& =-\operatorname{div}_{x}\left(\left|Y_{f}\right| \partial_{t} \mathbf{u}^{0}\right) \\
& +\partial_{t} p_{f} \int_{Y_{s}} \operatorname{div}_{y} \gamma^{p} d y+p_{f} \int_{Y_{s}} \operatorname{div}_{y} \partial_{t} \gamma^{p} d y \\
& +\sum_{i, j=1}^{3}\left(D_{x}\left(\partial_{t} \mathbf{u}^{0}\right)\right)_{i j} \int_{Y_{s}} \operatorname{div}\left(w^{i j}\right) d y+\sum_{i, j=1}^{3}\left(D_{x}\left(\mathbf{u}^{0}\right)\right)_{i j} \int_{Y_{s}} \operatorname{div}\left(\partial_{t} w^{i j}\right) d y \\
& p_{f}=0 \text { on }(0, T) \times \Gamma_{1} \\
& p_{f}=\mathcal{P}^{0} \quad \text { on }(0, T) \times \Gamma_{2} \\
& \int_{Y_{f}} \partial_{t} \mathbf{w}^{0} d y \cdot \nu=0 \quad \text { on }(0, T) \times \Gamma_{3}
\end{aligned}
$$


Now, extending the solution $p_{f}$ by reflection on $\Gamma_{3}$, and taking into account the regularity of the right hand side in (8.20), elliptic regularity theory, see e.g. [2], yields

$$
p_{f} \in H^{1}\left(0, T ; H^{3}(\Omega)\right),
$$

and (8.3) is proved. As a consequence, using the representation formula (8.12), we obtain

$$
\int_{Y_{f}} \partial_{t} \mathbf{w}^{0} d y \in H^{1}\left(0, T ; H^{2}(\Omega)\right) \subset L^{\infty}\left(Q_{T}\right) .
$$

Now, we remark that, due to (8.4), and (8.24), the effective transport velocity

$$
v^{H}(t, x)=\left(\left|Y_{f}\right|-\sum_{j=1}^{3} \int_{Y_{f}} d_{2} k(y) \nabla_{y} \lambda^{j}(y) e^{j} d y\right) \partial_{t} \mathbf{u}^{0}+\int_{Y_{f}} \partial_{t} \mathbf{w}^{0} d y
$$

lies in $L^{\infty}\left(Q_{T}\right)$, which proves (8.7).

We now still have to get the regularity (8.2) for $c_{2}^{0}$. For this, we write the effective problem for $c_{2}^{0}$ in the equivalent form:

$$
\begin{aligned}
& \left(\int_{Y} k(y) d y\right) \partial_{t} c_{2}^{0}-\operatorname{div}_{x}\left(d_{2}\left(\int_{Y} k(y) \Upsilon(y) d y\right) \nabla_{x} c_{2}^{0}\right) \\
& +\operatorname{div}_{x}\left\{\left[\left(\left|Y_{f}\right|-\sum_{j=1}^{3} \int_{Y_{f}} d_{2} k(y) \nabla_{y} \lambda^{j}(y) e^{j} d y\right) \partial_{t} \mathbf{u}^{0}+\int_{Y_{f}} \partial_{t} \mathbf{w}^{0} d y\right] c_{2}^{0}\right\} \\
& =\left|Y_{s}\right| g_{2}\left(c_{1}^{0}, c_{2}^{0}\right)+\left|Y_{f}\right| g_{3}\left(c_{2}^{0}\right) \\
& d_{2} \int_{Y} k(y) \Upsilon(y) d y \nabla_{x} c_{2}^{0} \cdot \nu \\
& -\left[\left(\left|Y_{f}\right|-\sum_{1}^{3} \int_{Y_{f}} d_{2} k(y) \nabla_{y} \lambda^{j}(y) e^{j} d y\right) \partial_{t} \mathbf{u}^{0}+\int_{Y_{f}} \partial_{t} \mathbf{w}^{0} d y\right] c_{2}^{0} \cdot \nu=0 \\
& \quad \text { on }(0, T) \times \Gamma_{3} \\
& c_{2}^{0}=c_{2 D} \quad \text { on }(0, T) \times\left(\Gamma_{1} \cup \Gamma_{2}\right) \\
& c_{2}^{0}(0, x)=c_{20} \quad \text { in } \Omega .
\end{aligned}
$$

Due to (8.11), and the Lipschitz property of the reaction terms, we have $\left|Y_{s}\right| g_{2}\left(c_{1}^{0}, c_{2}^{0}\right)+$ $\left|Y_{f}\right| g_{3}\left(c_{2}^{0}\right) \in L^{10 / 3}\left(Q_{T}\right)$. Since $v^{H} \in L^{\infty}\left(Q_{T}\right)$, we can again use the parabolic regularity theory, see Theorem 9.1, page 341 from [13], to obtain

$$
c_{2}^{0} \in W_{10 / 3}^{2,1}\left(Q_{T}\right) \subset L^{\infty}\left(Q_{T}\right) .
$$

For the last inclusion we used again Lemma 3.3, page 80 in [13].

9. Uniqueness for the homogenized problem. In this section, we prove uniqueness for weak solutions of the homogenized system given in (6.59)-(6.71).

THEOREM 9.1. The system (6.59)-(6.71) has a unique solution satisfying the regularity properties from Theorem 8.1.

Proof. We start our uniqueness proof by studying the stability of the variables describing the fluid-structure interaction, with respect to perturbations in the concentration $c_{1}^{0}$. Thus, let $\mathbf{u}_{j}^{0}, \mathbf{u}_{j}^{1}, \mathbf{w}_{j}^{0}, p_{f}^{j}, c_{1}^{j, 0}$, and $c_{2}^{j, 0}, j=1,2$, be two variational 
solutions of the homogenized system (6.59)-(6.71). We denote their differences by $\mathbf{u}^{0}=\mathbf{u}_{1}^{0}-\mathbf{u}_{2}^{0}, \mathbf{u}^{1}=\mathbf{u}_{1}^{1}-\mathbf{u}_{2}^{1}, \mathbf{w}^{0}=\mathbf{w}_{1}^{0}-\mathbf{w}_{2}^{0}, p_{f}=p_{f}^{1}-p_{f}^{2}, c_{1}^{0}=c_{1}^{1,0}-c_{1}^{2,0}$, and $c_{2}^{0}=c_{2}^{1,0}-c_{2}^{2,0}$. Then, with $\mathcal{A}_{j}=\mathcal{A}\left(\mathcal{F}\left(c_{1}^{j, 0}\right)\right)$, the equations (6.59)-(6.61) imply the following equations:

$$
\begin{aligned}
& \int_{\Omega} \int_{Y_{s}} \mathcal{A}_{1}\left(D_{x}\left(\mathbf{u}^{0}\right)+D_{y}\left(\mathbf{u}^{1}\right)\right): D_{x}(\varphi) d y d x-\int_{\Omega} \int_{Y_{f}} p_{f}(t, x) \operatorname{div}_{x} \varphi d y d x \\
& =\int_{\Omega} \int_{Y_{s}}\left(\mathcal{A}_{1}-\mathcal{A}_{2}\right)\left(D_{x}\left(\mathbf{u}_{2}^{0}\right)+D_{y}\left(\mathbf{u}_{2}^{1}\right)\right): D_{x}(\varphi) d y d x, \\
& \int_{\Omega} \int_{Y_{s}} \mathcal{A}_{1}\left(D_{x}\left(\mathbf{u}^{0}\right)+D_{y}\left(\mathbf{u}^{1}\right)\right) D_{y}(\psi) d y d x-\int_{\Omega} \int_{Y_{f}} p_{f}(t, x) \operatorname{div}_{y} \psi d y d x \\
& =\int_{\Omega} \int_{Y_{s}}\left(\mathcal{A}_{1}-\mathcal{A}_{2}\right)\left(D_{x}\left(\mathbf{u}_{2}^{0}\right)+D_{y}\left(\mathbf{u}_{2}^{1}\right)\right) D_{y}(\psi) d y d x, \\
& 2 \int_{\Omega} \int_{Y_{f}} D_{y}\left(\partial_{t} \mathbf{w}^{0}\right): D_{y}(\zeta) d x d y-\int_{\Omega} \int_{Y_{f}} p_{f} \operatorname{div}_{x} \zeta d y d x=0,
\end{aligned}
$$

for all $\varphi \in H^{1}(\Omega)^{3}, \varphi=0$ on $\Gamma_{3}, \psi \in L^{2}\left(\Omega, H_{p e r}^{1}(Y)\right)^{3}$, and $\zeta \in L^{2}\left(\Omega, H_{p e r}^{1}\left(Y_{f}\right)\right)^{3}, \operatorname{div}_{y} \zeta=$ 0 in $Y_{f} \times \Omega, \zeta=0$ on $\partial Y_{f} \backslash \partial Y$. at

Now, we take $\varphi=\partial_{t} \mathbf{u}^{0}, \psi=\partial_{t} \mathbf{u}^{1}$, and $\zeta=\partial_{t} \mathbf{w}^{0}$. After summing up, we arrive

$$
\begin{aligned}
& \int_{\Omega} \int_{Y_{s}} \mathcal{A}_{1}\left(D_{x}\left(\mathbf{u}^{0}\right)+D_{y}\left(\mathbf{u}^{1}\right)\right): \partial_{t}\left(D_{x}\left(\mathbf{u}^{0}\right)+D_{y}\left(\mathbf{u}^{1}\right)\right) \\
& +2 \int_{\Omega} \int_{Y_{f}}\left|D_{y}\left(\partial_{t} \mathbf{w}^{0}\right)\right|^{2} \\
& -\int_{\Omega} \int_{Y_{f}} p_{f}(t, x) \partial_{t}\left(\operatorname{div}_{x} \mathbf{u}^{0}+\operatorname{div}_{y} \mathbf{u}^{1}+\operatorname{div}_{x} \mathbf{w}^{0}\right)= \\
& \int_{\Omega} \int_{Y_{s}}\left(\mathcal{A}_{1}-\mathcal{A}_{2}\right)\left(D_{x}\left(\mathbf{u}_{2}^{0}\right)+D_{y}\left(\mathbf{u}_{2}^{1}\right)\right): \partial_{t}\left(D_{x}\left(\mathbf{u}^{0}\right)+D_{y}\left(\mathbf{u}^{1}\right)\right)
\end{aligned}
$$

Using now (6.62), which is valid also for the difference of solutions, and the periodicity property of $\mathbf{u}^{1}$, we obtain

$$
\begin{aligned}
& \int_{Y_{f}} \operatorname{div}_{x}\left(\partial_{t} \mathbf{u}^{0}+\partial_{t} \mathbf{w}^{0}\right)+\operatorname{div}_{y}\left(\partial_{t} \mathbf{u}^{1}\right) d y= \\
& \partial_{t}\left(\int_{Y_{s}} \operatorname{div}_{y} \mathbf{u}^{1} d y+\int_{Y_{f}} \operatorname{div}_{y} \mathbf{u}^{1} d y\right)=0
\end{aligned}
$$

Hence

$$
-\int_{\Omega} \int_{Y_{f}} p_{f}(t, x) \partial_{t}\left(\operatorname{div}_{x} \mathbf{u}^{0}+\operatorname{div}_{y} \mathbf{u}^{1}+\operatorname{div}_{x} \mathbf{w}^{0}\right)=0
$$

Furthermore, let $\mathcal{B}=D_{x}\left(\mathbf{u}^{0}\right)+D_{y}\left(\mathbf{u}^{1}\right)$. Then, we have

$$
\int_{\Omega} \int_{Y_{s}} \mathcal{A}_{1} \mathcal{B}: \partial_{t} \mathcal{B}=\frac{1}{2} \partial_{t}\left(\int_{\Omega} \int_{Y_{s}} \mathcal{A}_{1} \mathcal{B}: \mathcal{B}\right)-\frac{1}{2} \int_{\Omega} \int_{Y_{s}} \partial_{t}\left(\mathcal{A}_{1}\right) \mathcal{B}: \mathcal{B}
$$


Finally, for the last term on the right hand side in (9.1), we have:

$$
\begin{aligned}
& \int_{\Omega} \int_{Y_{s}}\left(\mathcal{A}_{1}-\mathcal{A}_{2}\right)\left(D_{x}\left(\mathbf{u}_{2}^{0}\right)+D_{y}\left(\mathbf{u}_{2}^{1}\right)\right): \partial_{t} \mathcal{B} d y d x= \\
& \partial_{t} \int_{\Omega} \int_{Y_{s}}\left(\mathcal{A}_{1}-\mathcal{A}_{2}\right)\left(D_{x}\left(\mathbf{u}_{2}^{0}\right)+D_{y}\left(\mathbf{u}_{2}^{1}\right)\right): \mathcal{B} d y d x \\
& -\int_{\Omega} \int_{Y_{s}} \partial_{t}\left(\mathcal{A}_{1}-\mathcal{A}_{2}\right)\left(D_{x}\left(\mathbf{u}_{2}^{0}\right)+D_{y}\left(\mathbf{u}_{2}^{1}\right)\right): \mathcal{B} d y d x \\
& -\int_{\Omega} \int_{Y_{s}}\left(\mathcal{A}_{1}-\mathcal{A}_{2}\right)\left(D_{x}\left(\partial_{t} \mathbf{u}_{2}^{0}\right)+D_{y}\left(\partial_{t} \mathbf{u}_{2}^{1}\right)\right): \mathcal{B} d y d x,
\end{aligned}
$$

and the following estimate holds:

$$
\begin{aligned}
& \left|\int_{0}^{t} \int_{\Omega} \int_{Y_{s}}\left(\mathcal{A}_{1}-\mathcal{A}_{2}\right)\left(D_{x}\left(\mathbf{u}_{2}^{0}\right)+D_{y}\left(\mathbf{u}_{2}^{1}\right)\right): \partial_{t} \mathcal{B} d y d x d \tau\right| \\
& \leq\left\|\mathcal{A}_{1}-\mathcal{A}_{2}\right\|_{L_{t}^{\infty} L_{x}^{3}}\left\{\left\|D_{x}\left(\mathbf{u}_{2}^{0}\right)\right\|_{L_{t}^{\infty} L_{x}^{6}}+\left\|D_{y}\left(\mathbf{u}_{2}^{1}\right)\right\|_{L_{t}^{\infty} L_{x}^{6} L_{y}^{2}}\right\}\|\mathcal{B}(t)\|_{L_{x}^{2} L_{y}^{2}} \\
& +\left\|\partial_{t}\left(\mathcal{A}_{1}-\mathcal{A}_{2}\right)\right\|_{L_{t}^{\infty} L_{x}^{3}} \int_{0}^{t}\left\{\left\|D_{x}\left(\mathbf{u}_{2}^{0}\right)\right\|_{L_{x}^{6}}(\tau)+\left\|D_{y}\left(\mathbf{u}_{2}^{1}\right)\right\|_{L_{x}^{6} L_{y}^{2}}(\tau)\right\} \times \\
& \times\|\mathcal{B}((\tau))\|_{L_{x}^{2} L_{y}^{2}} d \tau \\
& +\left\|\left(\mathcal{A}_{1}-\mathcal{A}_{2}\right)\right\|_{L_{t}^{\infty} L_{x}^{3}} \int_{0}^{t}\left\{\left\|D_{x}\left(\partial_{\tau} \mathbf{u}_{2}^{0}\right)\right\|_{L_{x}^{6}}(\tau)+\left\|D_{y}\left(\partial_{\tau} \mathbf{u}_{2}^{1}\right)\right\|_{L_{x}^{6} L_{y}^{2}}(\tau)\right\} \times \\
& \times\|\mathcal{B}((\tau))\|_{L_{x}^{2} L_{y}^{2}} d \tau,
\end{aligned}
$$

where we denoted by $\|\cdot\|_{L_{t}^{p} L_{x}^{q}}=\|\cdot\|_{L^{p}\left(0, t ; L^{q}(\Omega)\right)},\|\cdot\|_{L_{x}^{p} L_{y}^{q}}=\|\cdot\|_{L^{p}\left(\Omega ; L^{q}\left(Y_{s}\right)\right)}$, and $\|\cdot\|_{L_{t}^{p} L_{x}^{q} L_{y}^{r}}=\|\cdot\|_{L^{p}\left(0, t ; L^{q}\left(\Omega, L^{r}\left(Y_{s}\right)\right)\right)}$. After inserting (9.2), (9.3), (9.5) in (9.1), we obtain:

$$
\begin{aligned}
& \frac{1}{2} \int_{\Omega} \int_{Y_{s}} \mathcal{A}_{1}\left(D_{x}\left(\mathbf{u}^{0}\right)+D_{y}\left(\mathbf{u}^{1}\right)\right):\left(D_{x}\left(\mathbf{u}^{0}\right)+D_{y}\left(\mathbf{u}^{1}\right)\right) \\
& +2 \int_{0}^{t} \int_{\Omega} \int_{Y_{f}}\left|D_{y}\left(\partial_{t} \mathbf{w}^{0}\right)\right|^{2} \\
& \leq \frac{1}{2}\left\|\partial_{t} \mathcal{A}_{1}\right\|_{L_{t}^{\infty} L_{x}^{3}} \int_{0}^{t} \int_{\Omega} \int_{Y_{s}}\left|\left(D_{x}\left(\mathbf{u}^{0}\right)+D_{y}\left(\mathbf{u}^{1}\right)\right)(\tau)\right|^{2} d y d x d \tau \\
& +C\left\|\mathcal{A}_{1}-\mathcal{A}_{2}\right\|_{L_{t}^{\infty} L_{x}^{3}}\left\|\left(D_{x}\left(\mathbf{u}^{0}\right)+D_{y}\left(\mathbf{u}^{1}\right)\right)(t)\right\|_{L_{x}^{2} L_{y}^{2}}^{2} \\
& +C\left\|\partial_{t}\left(\mathcal{A}_{1}-\mathcal{A}_{2}\right)\right\|_{L_{t}^{\infty} L_{x}^{3}} \int_{0}^{t}\left\|\left(D_{x}\left(\mathbf{u}^{0}\right)+D_{y}\left(\mathbf{u}^{1}\right)\right)(\tau)\right\|_{L_{x}^{2} L_{y}^{2}} d \tau \\
& +C\left\|\mathcal{A}_{1}-\mathcal{A}_{2}\right\|_{L_{t}^{\infty} L_{x}^{3}} \int_{0}^{t}\left\|\left(D_{x}\left(\mathbf{u}^{0}\right)+D_{y}\left(\mathbf{u}^{1}\right)\right)(\tau)\right\|_{L_{x}^{2} L_{y}^{2}} d \tau
\end{aligned}
$$

Let $\mathcal{Y}(t)=\int_{0}^{t} \int_{\Omega} \int_{Y_{s}}\left|\left(D_{x}\left(\mathbf{u}^{0}\right)+D_{y}\left(\mathbf{u}^{1}\right)\right)(\tau)\right|^{2} d y d x d \tau$. Then (9.12) implies

$$
\begin{aligned}
& \frac{d}{d t} \mathcal{Y}(t)+2 \int_{0}^{t} \int_{\Omega} \int_{Y_{f}}\left|D_{y}\left(\partial_{t} \mathbf{w}^{0}\right)\right|^{2} \\
& \leq C \mathcal{Y}(t)+C\left\{\left\|\mathcal{A}_{1}-\mathcal{A}_{2}\right\|_{L_{t}^{\infty} L_{x}^{3}}+\left\|\partial_{t}\left(\mathcal{A}_{1}-\mathcal{A}_{2}\right)\right\|_{L_{t}^{\infty} L_{x}^{3}}\right\}
\end{aligned}
$$


Using Gronwall's inequality, we obtain for all $t \in(0, T)$

$$
\begin{aligned}
& \int_{\Omega} \int_{Y_{s}}\left|\left(D_{x}\left(\mathbf{u}^{0}\right)+D_{y}\left(\mathbf{u}^{1}\right)\right)(t)\right|^{2} d y d x \\
& \leq C\left\{\left\|\mathcal{A}_{1}-\mathcal{A}_{2}\right\|_{L^{\infty}\left(0, t ; L^{3}(\Omega)\right)}^{2}+\left\|\partial_{t}\left(\mathcal{A}_{1}-\mathcal{A}_{2}\right)\right\|_{L^{\infty}\left(0, t ; L^{3}(\Omega)\right)}^{2}\right\}
\end{aligned}
$$

where

$$
\begin{aligned}
\partial_{t}\left(\mathcal{A}_{1}-\mathcal{A}_{2}\right)= & \left(\frac{d \mathcal{A}}{d \mathcal{F}}\left(\mathcal{F}\left(c_{1}^{1,0}\right)\right)-\frac{d \mathcal{A}}{d \mathcal{F}}\left(\mathcal{F}\left(c_{1}^{2,0}\right)\right)\right) \partial_{t} \mathcal{F}\left(c_{1}^{1,0}\right) \\
& +\frac{d \mathcal{A}}{d \mathcal{F}}\left(\mathcal{F}\left(c_{1}^{2,0}\right)\right) \int_{0}^{t} \frac{d \mathcal{K}}{d t}(t-\tau)\left(\mathcal{F}\left(c_{1}^{1,0}\right)-\mathcal{F}\left(c_{1}^{2,0}\right)\right)(\tau) d \tau
\end{aligned}
$$

Using analogous techniques, in the following we obtain similar stability estimates for the time derivatives. We derive equations (6.59)-(6.61) with respect to time, and consider the equations satisfied by the differences $\partial_{t} \mathbf{u}^{0}=\partial_{t}\left(\mathbf{u}_{1}^{0}-\mathbf{u}_{2}^{0}\right), \partial_{t} \mathbf{u}^{1}=$ $\partial_{t}\left(\mathbf{u}_{1}^{1}-\mathbf{u}_{2}^{1}\right), \partial_{t} \mathbf{w}^{0}=\partial_{t}\left(\mathbf{w}_{1}^{0}-\mathbf{w}_{2}^{0}\right)$, and $\partial_{t} p_{f}=\partial_{t}\left(p_{f}^{1}-p_{f}^{2}\right)$ :

$$
\begin{aligned}
& \int_{\Omega} \int_{Y_{s}} \mathcal{A}_{1}\left(D_{x}\left(\partial_{t} \mathbf{u}^{0}\right)+D_{y}\left(\partial_{t} \mathbf{u}^{1}\right)\right): D_{x}(\varphi)-\int_{\Omega} \int_{Y_{f}} \partial_{t} p_{f}(t, x) \operatorname{div}_{x} \varphi \\
& =\int_{\Omega} \int_{Y_{s}}\left(\mathcal{A}_{1}-\mathcal{A}_{2}\right)\left(D_{x}\left(\partial_{t} \mathbf{u}_{2}^{0}\right)+D_{y}\left(\partial_{t} \mathbf{u}_{2}^{1}\right)\right): D_{x}(\varphi) \\
& -\int_{\Omega} \int_{Y_{s}}\left(\partial_{t} \mathcal{A}_{1}-\partial_{t} \mathcal{A}_{2}\right)\left(D_{x}\left(\partial_{t} \mathbf{u}_{1}^{0}\right)+D_{y}\left(\partial_{t} \mathbf{u}_{1}^{1}\right)\right): D_{x}(\varphi) \\
& -\int_{\Omega} \int_{Y_{s}} \partial_{t} \mathcal{A}_{2}\left(D_{x}\left(\mathbf{u}^{0}\right)+D_{y}\left(\mathbf{u}^{1}\right)\right): D_{x}(\varphi), \\
& \int_{\Omega} \int_{Y_{s}} \mathcal{A}_{1}\left(D_{x}\left(\partial_{t} \mathbf{u}^{0}\right)+D_{y}\left(\partial_{t} \mathbf{u}^{1}\right)\right) D_{y}(\psi) d y d x-\int_{\Omega} \int_{Y_{f}} \partial_{t} p_{f}(t, x) \operatorname{div}_{y} \psi \\
& =\int_{\Omega} \int_{Y_{s}}\left(\mathcal{A}_{1}-\mathcal{A}_{2}\right)\left(D_{x}\left(\partial_{t} \mathbf{u}_{2}^{0}\right)+D_{y}\left(\partial_{t} \mathbf{u}_{2}^{1}\right)\right) D_{y}(\psi) \\
& -\int_{\Omega} \int_{Y_{s}}\left(\partial_{t} \mathcal{A}_{1}-\partial_{t} \mathcal{A}_{2}\right)\left(D_{x}\left(\mathbf{u}_{1}^{0}\right)+D_{y}\left(\mathbf{u}_{1}^{1}\right)\right) D_{y}(\psi) \\
& -\int_{\Omega} \int_{Y_{s}} \partial_{t} \mathcal{A}_{2}\left(D_{x}\left(\mathbf{u}^{0}\right)+D_{y}\left(\mathbf{u}^{1}\right)\right) D_{y}(\psi), \\
& 2 \int_{\Omega} \int_{Y_{f}} D_{y}\left(\partial_{t t} \mathbf{w}^{0}\right): D_{y}(\zeta)-\int_{\Omega} \int_{Y_{f}} \partial_{t} p_{f} \operatorname{div}_{x} \zeta=0 .
\end{aligned}
$$

Now, we take $\varphi=\partial_{t t} \mathbf{u}^{0} \in L^{2}\left(0, T ; H^{1}(\Omega)\right)^{3}, \psi=\partial_{t t} \mathbf{u}^{1} \in L^{2}\left(Q_{T} ; H_{p e r}^{1}\left(Y_{s}\right)\right)$, and 
$\zeta=\partial_{t t} \mathbf{w}^{0} \in L^{2}\left(Q_{T} ; H_{p e r}^{1}\left(Y_{f}\right)\right)$. After summing up, we arrive at

$$
\begin{aligned}
& \int_{\Omega} \int_{Y_{s}} \mathcal{A}_{1}\left(D_{x}\left(\partial_{t} \mathbf{u}^{0}\right)+D_{y}\left(\partial_{t} \mathbf{u}^{1}\right)\right): \partial_{t}\left(D_{x}\left(\partial_{t} \mathbf{u}^{0}\right)+D_{y}\left(\partial_{t} \mathbf{u}^{1}\right)\right) \\
& +2 \int_{\Omega} \int_{Y_{f}}\left|D_{y}\left(\partial_{t t} \mathbf{w}^{0}\right)\right|^{2} \\
& -\int_{\Omega} \int_{Y_{f}} \partial_{t} p_{f}(t, x) \partial_{t t}\left(\operatorname{div}_{x} \mathbf{u}^{0}+\operatorname{div}_{y} \mathbf{u}^{1}+\operatorname{div}_{x} \mathbf{w}^{0}\right) \\
& =\int_{\Omega} \int_{Y_{s}}\left(\mathcal{A}_{1}-\mathcal{A}_{2}\right)\left(D_{x}\left(\partial_{t} \mathbf{u}_{2}^{0}\right)+D_{y}\left(\partial_{t} \mathbf{u}_{2}^{1}\right)\right): \partial_{t}\left(D_{x}\left(\partial_{t} \mathbf{u}^{0}\right)+D_{y}\left(\partial_{t} \mathbf{u}^{1}\right)\right) \\
& -\int_{\Omega} \int_{Y_{s}}\left(\partial_{t} \mathcal{A}_{1}-\partial_{t} \mathcal{A}_{2}\right)\left(D_{x}\left(\mathbf{u}_{1}^{0}\right)+D_{y}\left(\mathbf{u}_{1}^{1}\right)\right):\left(D_{x}\left(\partial_{t t} \mathbf{u}^{0}\right)+D_{y}\left(\partial_{t t} \mathbf{u}^{1}\right)\right) \\
& -\int_{\Omega} \int_{Y_{s}} \partial_{t} \mathcal{A}_{2}\left(D_{x}\left(\mathbf{u}^{0}\right)+D_{y}\left(\mathbf{u}^{1}\right)\right):\left(D_{x}\left(\partial_{t t} \mathbf{u}^{0}\right)+D_{y}\left(\partial_{t t} \mathbf{u}^{1}\right)\right),
\end{aligned}
$$

Once more, we can use (6.62), to conclude that

$$
\int_{Y_{f}} \operatorname{div}_{x}\left(\partial_{t t} \mathbf{u}^{0}+\partial_{t t} \mathbf{w}^{0}\right)+\operatorname{div}_{y}\left(\partial_{t t} \mathbf{u}^{1}\right) d y=0
$$

and thus

$$
-\int_{\Omega} \int_{Y_{f}} \partial_{t} p_{f}(t, x) \partial_{t t}\left(\operatorname{div}_{x} \mathbf{u}^{0}+\operatorname{div}_{y} \mathbf{u}^{1}+\operatorname{div}_{x} \mathbf{w}^{0}\right)=0 .
$$

We estimate now the terms on the right hand side of (9.10) analogously to the right hand side in (9.1), and obtain

$$
\begin{aligned}
& \int_{\Omega} \int_{Y_{s}} \mathcal{A}_{1}\left(D_{x}\left(\partial_{t} \mathbf{u}^{0}\right)+D_{y}\left(\partial_{t} \mathbf{u}^{1}\right)\right):\left(D_{x}\left(\partial_{t} \mathbf{u}^{0}\right)+D_{y}\left(\partial_{t} \mathbf{u}^{1}\right)\right) \\
& +\int_{0}^{t} \int_{\Omega} \int_{Y_{f}}\left|D_{y}\left(\partial_{t t} \mathbf{w}^{0}\right)\right|^{2} \\
& \leq C \int_{0}^{t} \int_{\Omega} \int_{Y_{s}}\left|\left(D_{x}\left(\partial_{t} \mathbf{u}^{0}\right)+D_{y}\left(\partial_{t} \mathbf{u}^{1}\right)\right)(\tau)\right|^{2} d y d x d \tau \\
& +C \int_{0}^{t} \int_{\Omega} \int_{Y_{s}}\left|\left(D_{x}\left(\mathbf{u}^{0}\right)+D_{y}\left(\mathbf{u}^{1}\right)\right)(\tau)\right|^{2} d y d x d \tau \\
& +C \int_{\Omega} \int_{Y_{s}}\left|\left(D_{x}\left(\mathbf{u}^{0}\right)+D_{y}\left(\mathbf{u}^{1}\right)\right)\right|^{2} d y d x \\
& +C\left\{\left\|\mathcal{A}_{1}-\mathcal{A}_{2}\right\|_{L_{t}^{\infty} L_{x}^{3}}^{2}+\left\|\partial_{t}\left(\mathcal{A}_{1}-\mathcal{A}_{2}\right)\right\|_{L_{t}^{\infty} L_{x}^{3}}^{2}+\left\|\partial_{t t}\left(\mathcal{A}_{1}-\mathcal{A}_{2}\right)\right\|_{L_{t}^{\infty} L_{x}^{3}}^{2}\right\}
\end{aligned}
$$

Using now (9.8), and Gronwall's inequality, we obtain for all $t \in(0, T)$

$$
\begin{aligned}
& \left.\| D_{x}\left(\mathbf{u}^{0}\right)+D_{y}\left(\mathbf{u}^{1}\right)\right)(t) \|_{L^{2}\left(\Omega \times Y_{s}\right)} \\
+ & \left.\| D_{x}\left(\partial_{t} \mathbf{u}^{0}\right)+D_{y}\left(\partial_{t} \mathbf{u}^{1}\right)\right)(t) \|_{L^{2}\left(\Omega \times Y_{s}\right)} \\
\leq & C\left\{\left\|\mathcal{A}_{1}-\mathcal{A}_{2}\right\|_{L^{\infty}\left(0, t ; L^{3}(\Omega)\right)}+\left\|\partial_{t}\left(\mathcal{A}_{1}-\mathcal{A}_{2}\right)\right\|_{L^{\infty}\left(0, t ; L^{3}(\Omega)\right)}\right\} \\
+ & C\left\|\partial_{t t}\left(\mathcal{A}_{1}-\mathcal{A}_{2}\right)\right\|_{L^{\infty}\left(0, t ; L^{3}(\Omega)\right)} \\
\leq & C\left\|c_{1}^{0}\right\|_{L^{1}\left(0, t ; L^{3}(\Omega)\right)} .
\end{aligned}
$$


Estimates (9.12), and (9.13) now imply

$$
\left\|\partial_{t} \mathbf{u}^{0}\right\|_{L^{2}\left(Q_{t}\right)}+\left\|\int_{Y_{f}} \partial_{t} \mathbf{w}^{0} d y\right\|_{L^{2}\left(Q_{t}\right)} \leq C\left\|c_{1}^{0}\right\|_{L^{1}\left(0, t ; L^{3}(\Omega)\right)} .
$$

The next step in the proof is to study the stability of the concentration $c_{2}^{0}$ with respect to the concentration $c_{1}^{0}$. Let us denote by $k_{0}=\int_{Y} k(y) d y$. Then the difference $c_{2}^{0}=c_{2}^{1,0}-c_{2}^{2,0}$ satisfies the following equation

$$
\begin{aligned}
& \int_{0}^{t} \int_{\Omega} k_{0} \partial_{t} c_{2}^{0} \psi+\int_{0}^{t} \int_{\Omega} d_{2} \int_{Y} k(y) \Upsilon(y) d y \nabla_{x} c_{2}^{0} \nabla_{x} \psi d x \\
& -\int_{0}^{t} \int_{\Omega} v_{1}^{H} c_{2}^{0} \nabla_{x} \psi d x-\int_{0}^{t} \int_{\Omega}\left(v_{1}^{H}-v_{2}^{H}\right) c_{2}^{2,0} \nabla_{x} \psi d x \\
& =\int_{0}^{t} \int_{\Omega}\left|Y_{s}\right|\left(g_{2}\left(c_{1}^{1,0}, c_{2}^{1,0}\right)-g_{2}\left(c_{1}^{2,0}, c_{2}^{2,0}\right)\right) \psi d x \\
& +\int_{0}^{t} \int_{\Omega}\left|Y_{f}\right|\left(g_{3}\left(c_{2}^{1,0}\right)-g_{3}\left(c_{2}^{2,0}\right)\right) \psi d x,
\end{aligned}
$$

where the effective transport velocities $v_{i}^{H}, i=1,2$, are given by

$$
v_{i}^{H}(t, x)=\left(\left|Y_{f}\right|-\sum_{j=1}^{3} \int_{Y_{f}} d_{2} k(y) \nabla_{y} \lambda^{j}(y) e^{j} d y\right) \partial_{t} \mathbf{u}_{i}^{0}+\int_{Y_{f}} \partial_{t} \mathbf{w}_{i}^{0} d y
$$

see also (8.6). Now, we insert in (9.15) as test function $\psi=c_{2}^{0}$, and obtain

$$
\begin{aligned}
& \frac{1}{2} \int_{\Omega} k_{0}\left|c_{2}^{0}(t)\right|^{2} d x+\int_{0}^{t} \int_{\Omega} d_{2} \int_{Y} k(y) \Upsilon(y) d y\left|\nabla_{x} c_{2}^{0}\right|^{2} d x d \tau \\
& =\int_{0}^{t} \int_{\Omega} v_{1}^{H} c_{2}^{0} \nabla_{x} c_{2}^{0} d x d \tau+\int_{0}^{t} \int_{\Omega}\left(v_{1}^{H}-v_{2}^{H}\right) c_{2}^{2,0} \nabla_{x} c_{2}^{0} d x d \tau \\
& +\int_{0}^{t} \int_{\Omega}\left|Y_{s}\right|\left(g_{2}\left(c_{1}^{1,0}, c_{2}^{1,0}\right)-g_{2}\left(c_{1}^{2,0}, c_{2}^{2,0}\right)\right) c_{2}^{0} d x d \tau \\
& +\int_{0}^{t} \int_{\Omega}\left|Y_{f}\right|\left(g_{3}\left(c_{2}^{1,0}\right)-g_{3}\left(c_{2}^{2,0}\right)\right) c_{2}^{0} d x d \tau
\end{aligned}
$$

Let us now estimate the terms on the right hand side of (9.16).

$$
\begin{aligned}
& \left|\int_{0}^{t} \int_{\Omega} v_{1}^{H} c_{2}^{0} \nabla_{x} c_{2}^{0} d x d \tau\right| \leq\left\|v_{1}^{H}\right\|_{L^{\infty}\left(Q_{t}\right)}\left\|c_{2}^{0}\right\|_{L^{2}\left(Q_{t}\right)}|| \nabla_{x} c_{2}^{0} \|_{L^{2}\left(Q_{t}\right)} \\
& \leq \delta\left\|\nabla_{x} c_{2}^{0}\right\|_{L^{2}\left(Q_{t}\right)}^{2}+C(\delta)\left\|c_{2}^{0}\right\|_{L^{2}\left(Q_{t}\right)}^{2} \\
& \left|\int_{0}^{t} \int_{\Omega}\left(v_{1}^{H}-v_{2}^{H}\right) c_{2}^{2,0} \nabla_{x} c_{2}^{0} d x d \tau\right| \\
& \leq\left\|v_{1}^{H}-v_{2}^{H}\right\|_{L^{2}\left(Q_{t}\right)}\left\|c_{2}^{0}\right\|_{L^{\infty}\left(Q_{t}\right)}\left\|\nabla_{x} c_{2}^{0}\right\|_{L^{2}\left(Q_{t}\right)} \\
& \leq \delta\left\|\nabla_{x} c_{2}^{0}\right\|_{L^{2}\left(Q_{t}\right)}^{2}+C(\delta)\left\|v_{1}^{H}-v_{2}^{H}\right\|_{L^{2}\left(Q_{t}\right)}^{2} \\
& \left|\int_{0}^{t} \int_{\Omega}\right| Y_{s}\left|\left(g_{2}\left(c_{1}^{1,0}, c_{2}^{1,0}\right)-g_{2}\left(c_{1}^{2,0}, c_{2}^{2,0}\right)\right) c_{2}^{0} d x d \tau\right| \\
& \leq C\left(\left\|c_{1}^{0}\right\|_{L^{2}\left(Q_{t}\right)}^{2}+\left\|c_{2}^{0}\right\|_{L^{2}\left(Q_{t}\right)}^{2}\right) \\
& \left|\int_{0}^{t} \int_{\Omega}\right| Y_{f}\left|\left(g_{3}\left(c_{2}^{1,0}\right)-g_{3}\left(c_{2}^{2,0}\right)\right) c_{2}^{0} d x d \tau\right| \leq C\left\|c_{2}^{0}\right\|_{L^{2}\left(Q_{t}\right)}^{2}
\end{aligned}
$$


To estimate the difference $\left\|v_{1}^{H}-v_{2}^{H}\right\|_{L^{2}\left(Q_{t}\right)}$, use the stability estimate (9.14) to obtain

$$
\left\|v_{1}^{H}-v_{2}^{H}\right\|_{L^{\infty}\left(0, t ; L^{2}(\Omega)\right)} \leq C\left\|c_{1}^{0}\right\|_{L^{1}\left(0, t ; L^{3}(\Omega)\right)}
$$

Inserting now (9.17)-(9.20), and (9.21) in (9.16) leads to the following estimate

$$
\begin{aligned}
& \int_{\Omega} k_{0}\left|c_{2}^{0}(t)\right|^{2} d x+\int_{0}^{t} \int_{\Omega} d_{2} \int_{Y} k(y) \Upsilon(y) d y\left|\nabla_{x} c_{2}^{0}\right|^{2} d x d \tau \\
& \leq C\left(\left\|c_{2}^{0}\right\|_{L^{2}\left(Q_{t}\right)}^{2}+\left\|c_{1}^{0}\right\|_{L^{2}\left(Q_{t}\right)}^{2}+\left\|c_{1}^{0}\right\|_{L^{1}\left(0, t ; L^{3}(\Omega)\right)}^{2}\right)
\end{aligned}
$$

Now applying Gronwall's inequality yields the following stability estimate

$$
\begin{aligned}
\left\|c_{2}^{0}\right\|_{L^{\infty}\left(0, t ; L^{2}(\Omega)\right)} & \leq C\left(\left\|c_{1}^{0}\right\|_{L^{2}\left(Q_{t}\right)}^{2}+\left\|c_{1}^{0}\right\|_{L^{1}\left(0, t ; L^{3}(\Omega)\right)}^{2}\right) \\
& \leq C\left\|c_{1}^{0}\right\|_{L^{2}\left(0, t ; L^{3}(\Omega)\right)}^{2} .
\end{aligned}
$$

Finally, we consider the equation for the difference $c_{1}^{0}=c_{1}^{1,0}-c_{1}^{2,0}$. Using (6.67), we obtain

$$
\begin{aligned}
& \left|Y_{s}\right| \int_{0}^{t} \int_{\Omega} \partial_{t} c_{1}^{0} \varphi d x d \tau+\int_{0}^{t} \int_{\Omega} d_{1} \beta \nabla c_{1}^{0} \nabla \varphi d x d \tau= \\
& \left|Y_{s}\right| \int_{0}^{t} \int_{\Omega}\left(g_{1}\left(c_{1}^{1,0}, c_{2}^{1,0}\right)-g_{1}\left(c_{1}^{2,0}, c_{2}^{2,0}\right)\right) \varphi d x d \tau
\end{aligned}
$$

Testing with the $\varphi=c_{1}^{0}$ leads to

$$
\int_{\Omega}\left|c_{1}^{0}(t)\right|^{2} d x+\int_{0}^{t} \int_{\Omega} d_{1} \beta\left|\nabla c_{1}^{0}\right|^{2} d x d \tau=C\left(|| c_{1}^{0}\left\|_{L^{2}\left(Q_{t}\right)}^{2}+\right\| c_{2}^{0} \|_{L^{2}\left(Q_{t}\right)}^{2}\right)
$$

Inserting (9.23) in (9.25), and using interpolation, we obtain

$$
\int_{\Omega}\left|c_{1}^{0}(t)\right|^{2} d x+\int_{0}^{t} \int_{\Omega} d_{1} \beta\left|\nabla c_{1}^{0}\right|^{2} d x d \tau \leq C|| c_{1}^{0} \|_{L^{2}\left(Q_{t}\right)}^{2},
$$

which then, by Gronwall's inequality, leads to

$$
c_{1}^{0}=0 \text { a. e. on }(0, T) \times \Omega .
$$

The result (9.27), together with the stability estimates (9.12), (9.13), and (9.23) prove the uniqueness of the variational solution to the homogenized system (6.59)-(6.71).

Acknowledgments. This work was partly done when A.M. was on the sabbatical leave at IWR and Institut für Angewandte Mathematik, Universität Heidelberg, October 1, 2005 - March 31, 2006 and then completed during subsequent visits in April 2007, May 2008 and February 2010.

\section{REFERENCES}

[1] E. Acerbi, V. Chiado Piat, G. Dal Maso, D. Percivale An extension theorem from connected sets, and homogenization in general periodic domains. Nonlinear Anal., TMA, 18 (1992), pp. 481-496.

[2] S. Agmon, A. Douglis, L. Nirenberg Estimates near the boundary for solutions of elliptic partial differential equations satisfying general boundary conditions. Comm. Pure Appl. Math. 12 (1959), pp. 623-727. 
[3] G. Allaire, Homogenization and two-scale convergence, SIAM J. Math. Anal. 23 (1992), pp. $1482-1518$.

[4] A. Bourgeat, S. Luckhaus, A. Mikelić Convergence of the homogenization process for a double-porosity model of immiscible two-phase flow. SIAM J. Math. Anal. 27 (1996), pp. $1520-1543$.

[5] D. Cioranescu, A. Damlamian, G. Griso The periodic unfolding method in homogenization. SIAM J. Math. Anal. 40 (2008), pp. 1585-1620.

[6] T. Clopeau, J.L. Ferrin, R.P. Gilbert, A. Mikelić Homogenizing the acoustic properties of the seebed: Part II, Mathematical and Computer Modelling 33 (2001), pp. 821-841.

[7] J.L. Ferrin, A. Mikelić Homogenizing the acoustic properties of a porous matrix containing an incompressible inviscid fluid, Math. Methods Appl. Sci. 26 (2003), pp. 831-859.

[8] R.P. Gilbert, A. Mikelić Homogenizing the acoustic properties of the seebed: Part I, Nonlinear Analysis 40 (2000), pp. 185-212.

[9] O. Iliev, A. Mikelić, P. Popov On upscaling certain flows in deformable porous media, Multiscale Model. Simul. 7 (2008), pp. 93-123.

[10] W. Jäger, A. Mikelić, M. Neuss-Radu Analysis of Differential Equations Modelling the Reactive Flow through a Deformable System of Cells. Arch. Rational Mech. Anal. 192 (2009), pp. 331-374.

[11] A. KLARBRING Examples of non-uniqueness and non-existence of solutions to quasistatic contact problems with friction. Ingen. Archiv 60 (1990), pp. 529-541.

[12] O. A. Ladyzenskaja, N. N. Ural'Ceva Linear and quasi-linear elliptic equations, Academic Press, New York and London (1968).

[13] O. A. Ladyzenskaja, V. A. Solonnikov, N. N. Ural'Ceva Linear and quasi-linear equations of parabolic type, American Mathematical Society, Providence, Rhode Island, (1968).

[14] O. A. Oleinik, A. M. Shamaev, A. G. Yosifian Mathematical Problems in Elasticity and Homogenization, North Holland, Amsterdam, London, New York, Tokyo, (1992)

[15] J. Seebach, H.-J. Mädler, B. Wojciak-Stothard, H.-J. Schnittler Tyrosine phosphorylation and the small GTPase rac cross-talk in regulation of endothelial barrier function, Thromb Haemost: Cell Signalling and Vessel Remodelling, 94 (2005), pp. 620-629.

[16] I. Tolstoy, ED. Acoustics, elasticity, and thermodynamics of porous media, Twenty-one papers by M. A. Biot, Acoustical Society of America, New York (1992).

[17] R. Temam Navier Stokes Equations, North Holland, Amsterdam (1977). 